\title{
EXISTENCE OF GLOBAL WEAK SOLUTIONS TO SOME REGULARIZED KINETIC MODELS FOR DILUTE POLYMERS*
}

\author{
JOHN W. BARRETT ${ }^{\dagger}$ AND ENDRE SÜLI ${ }^{\ddagger}$
}

\begin{abstract}
We study the existence of global-in-time weak solutions to a coupled microscopicmacroscopic bead-spring model which arises from the kinetic theory of dilute solutions of polymeric liquids with noninteracting polymer chains. The model consists of the unsteady incompressible Navier-Stokes equations in a bounded domain $\Omega \subset \mathbb{R}^{d}, d=2$ or 3 , for the velocity and the pressure of the fluid, with an elastic extra-stress tensor as the right-hand side in the momentum equation. The extra-stress tensor stems from the random movement of the polymer chains and is defined through the associated probability density function which satisfies a Fokker-Planck-type parabolic equation, a crucial feature of which is the presence of a center-of-mass diffusion term. The anisotropic Friedrichs mollifiers, which naturally arise in the course of the derivation of the model in the Kramers expression for the extra-stress tensor and in the drag term in the Fokker-Planck equation, are replaced by isotropic Friedrichs mollifiers. We establish the existence of global-in-time weak solutions to the model for a general class of spring-force-potentials including, in particular, the widely used finitely extensible nonlinear elastic (FENE) potential. We justify also, through a rigorous limiting process, certain classical reductions of this model appearing in the literature which exclude the center-of-mass diffusion term from the Fokker-Planck equation on the grounds that the diffusion coefficient is small relative to other coefficients featuring in the equation. In the case of a corotational drag term we perform a rigorous passage to the limit as the Friedrichs mollifiers in the Kramers expression and the drag term converge to identity operators.
\end{abstract}

Key words. polymeric flow models, existence of weak solutions, Navier-Stokes equations, Fokker-Planck equations, finitely extensible nonlinear elastic

AMS subject classifications. 76D03, 82C31, 82D60

DOI. $10.1137 / 060666810$

1. Introduction. This paper is concerned with the question of existence of global weak solutions to a system of nonlinear partial differential equations which arises from the kinetic theory of dilute polymer solutions. The solvent is an incompressible, viscous, isothermal Newtonian fluid confined to a bounded open set $\Omega \subset \mathbb{R}^{d}$, $d=2$ or 3 , with boundary $\partial \Omega$. For the sake of simplicity of presentation, we shall suppose that $\Omega$ has solid boundary $\partial \Omega$; the velocity field $u$ will then satisfy the no-slip boundary condition $u=0$ on $\partial \Omega$. The polymer chains which are suspended in the solvent are assumed not to interact with each other. The conservation of momentum and mass equations for the solvent then have the form of the incompressible NavierStokes equations in which the elastic extra-stress tensor $\underset{\sim}{\tau}$ (i.e., the polymeric part of the Cauchy stress tensor) appears as a source term:

Find $\underset{\sim}{u}:(\underset{\sim}{x}, t) \in \mathbb{R}^{d+1} \mapsto \underset{\sim}{u}(\underset{\sim}{x}, t) \in \mathbb{R}^{d}$ and $p:(\underset{\sim}{x}, t) \in \mathbb{R}^{d+1} \mapsto p(\underset{\sim}{x}, t) \in \mathbb{R}$ such

*Received by the editors August 3, 2006; accepted for publication (in revised form) February 5, 2007; published electronically June 29, 2007.

http://www.siam.org/journals/mms/6-2/66681.html

${ }^{\dagger}$ Department of Mathematics, Imperial College London, London SW7 2AZ, UK (j.barrett@ imperial.ac.uk).

${ }^{\ddagger}$ OUCL, University of Oxford, Parks Road, Oxford OX1 3QD, UK (endre.suli@comlab.ox.ac.uk). 
that

$$
\begin{array}{rlrl}
\frac{\partial u}{\partial t}+\left(\underset{\sim}{u} \cdot \underset{\sim}{\nabla_{x}}\right) \underset{\sim}{u}-\nu \Delta_{x} \underset{\sim}{u}+\underset{\sim}{\nabla_{x} p}=\underset{\sim}{\nabla_{x} \cdot \underset{\sim}{\tau}} & & \text { in } \Omega \times(0, T], \\
\nabla_{x} \cdot \underset{\sim}{u}=0 & & \text { in } \Omega \times(0, T], \\
\underset{\sim}{u}=0 & & \text { on } \partial \Omega \times(0, T], \\
\underset{\sim}{u}(\underset{\sim}{x}, 0) & ={\underset{\sim}{0}}_{0}(\underset{\sim}{x}) & & \forall \underset{\sim}{x} \in \Omega,
\end{array}
$$

where $u$ is the velocity field, $p$ is the pressure of the fluid, and $\nu \in \mathbb{R}_{>0}$ is the viscosity of the solvent. For the sake of simplicity, we shall assume that there are no body forces present: the presence of a body force $\underset{\sim}{f} \in L^{2}\left(\mathbb{R},\left[H^{-1}(\Omega)\right]^{d}\right)$ on the right-hand side of (1.1a) would not cause any particular technical complications. The extra-stress tensor $\tau$ is defined as the second moment of $\psi$, the probability density function of the (random) conformation vector of the polymer molecules. As will be seen below, the Kolmogorov equation satisfied by $\psi$ is a Fokker-Planck-type second-order parabolic equation whose transport coefficients depend on the velocity field $u$.

Polymer solutions exhibit a range of non-Newtonian flow properties: in particular, the stress endured by a fluid element depends upon the history of deformations experienced by that element. Thereby, rheological properties of non-Newtonian fluids are governed by the flow-induced evolution of their internal microstructure. Following Keunings [16], a relevant feature of the microstructure is the conformation of the macromolecules, i.e., their orientation and the degree of stretching they experience. From the macroscopic viewpoint it is only the statistical distribution of conformations that matters: the macroscopic stress carried by each fluid element is governed by the distribution of polymer conformations within that element. Motivated by this observation, kinetic theories of polymeric fluids ignore quantum mechanical and atomistic effects and focus on "coarse-grained" models of the polymeric conformations. Depending on the level of coarse graining, one may arrive at a hierarchy of kinetic models. For example, a dilute solution of linear polymers in a Newtonian solvent can be described in some detail by the freely jointed bead-rod Kramers chain, which comprises a number of beads (of the order of 100) connected by rigid linear segments. A coarser model of the same polymer is the freely jointed bead-spring chain, a Rouse chain, consisting of a smaller number of beads (of the order of 10) connected linearly by entropic springs. A coarser model still is the dumbbell model which involves two beads connected by a spring [5]. As has been emphasized by Keunings [16], such coarse-grained models are not meant to capture the detailed structure of the polymer. Rather, they are intended to describe, in more or less detail, the evolution of polymer conformations in a macroscopic flow.

Many of the interesting properties of dilute polymer solutions can be understood by modelling them as suspensions of simple coarse-grained objects (viz. dumbbells) in a Newtonian fluid. This paper is devoted to the mathematical analysis of dumbbell models which are nonlinearly coupled Navier-Stokes-Fokker-Planck systems of partial differential equations: from the technical viewpoint these relatively simple models already exemplify many of the analytical difficulties which are encountered in the study of more complex models.

Since our model problem differs in slight yet crucial details from classical beadspring models, here we provide a brief overview of the derivation of the model. Some of the key steps have been stimulated by the arguments put forward in Schieber [26] and in Chapter 1 of the recent doctoral thesis of Lozinski [19]; see also Lozinski, 


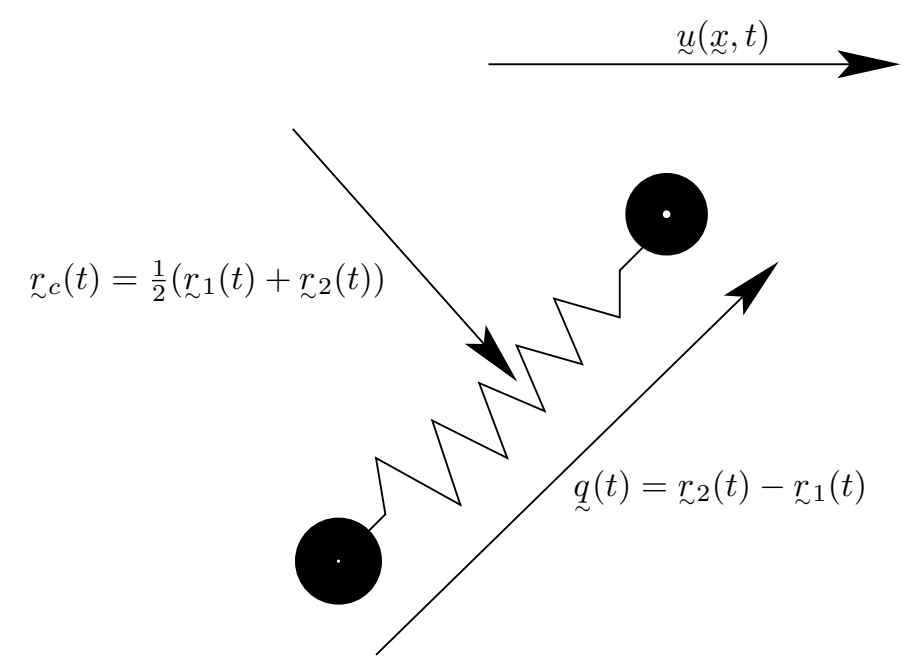

FIG. 1.1. Noninteracting polymer chains, immersed into an incompressible Newtonian solvent with flow velocity $\underset{\sim}{u}$, are modelled by using dumbbells, each dumbbell representing a polymer chain. A dumbbell is a pair of beads, with centers of mass located, with respect to some fixed origin, respectively, at $\underset{\sim}{\underset{\sim}{r}}(t)$ and $\underset{\sim}{\underset{2}{2}(t)}$ at time $t \geq 0$, connected with an elastic spring. The dumbbell is characterized by the position $\underset{\sim}{\sim} c(t)=\frac{1}{2}\left(\underset{\sim}{\sim} 1(t)+{\underset{\sim}{\sim} 2}_{2}(t)\right)$ of its center of mass and its elongation vector $\underset{\sim}{q}(t)=\underset{\sim}{r} 2(t)-{\underset{\sim}{r}}_{1}(t)$.

Owens, and Fang [21]. For related discussion on the derivation of kinetic polymer models we refer the reader to the books by Öttinger [22] and Bird et al. [5].

Let us denote by $\underset{\sim}{\underset{ }{i}}(t) \in \mathbb{R}^{d}, i=1,2$, the position vectors of the centers of mass of the two beads in the dumbbell at time $t \geq 0$ with respect to some fixed origin. The center of mass of the dumbbell is then located at $\underset{\sim}{\underset{r}{r}}(t):=\frac{1}{2}\left(\underset{\sim}{\underset{1}{r}}(t)+{\underset{\sim}{2}}_{2}(t)\right)$. We define the elongation vector (or end-to-end vector) of the molecule at time $t$ by $\underset{\sim}{q}(t)=\underset{\sim}{r} 2(t)-\underset{\sim}{r}(t)$; see Figure 1.1. The elongation vector $\underset{\sim}{q}(t)$ is assumed to be confined to a balanced convex open set $D \subset \mathbb{R}^{d}$; the term balanced means that $0 \in D$, and $-\underset{\sim}{q} \in D$ whenever $\underset{\sim}{q} \in D$. Typically, $D$ is an open $d$-dimensional ball of fixed radius $Q_{\max }$, an ellipse with fixed half-axes, or the whole of $\mathbb{R}^{d}$.

Assuming that each bead has mass $m$, the spring is massless, and in the absence of external forces, Langevin's equation from statistical mechanics states that

$$
\begin{aligned}
& m \mathrm{~d} v_{\sim}+\zeta \underset{\sim}{v} v_{1} \mathrm{~d} t=\underset{\sim}{B} 1 \mathrm{~d} t+\underset{\sim}{F}\left(\underset{\sim}{r_{2}}-\underset{\sim}{r_{1}}\right) \mathrm{d} t \\
& \text { and } \quad m \underset{\sim}{\mathrm{d} v_{2}}+\zeta \underset{\sim}{v} \underset{\sim}{v} \mathrm{~d} t=\underset{\sim}{B} \mathrm{~B} \mathrm{~d} t+\underset{\sim}{F}\left(\underset{\sim}{r_{1}}-\underset{\sim}{r_{2}}\right) \mathrm{d} t \text {, }
\end{aligned}
$$

where $\zeta$ is a friction coefficient, $k$ is the Boltzmann constant, $\mu$ is the absolute temperature, and ${\underset{\sim}{v}}_{i}$ is the velocity of the $i$ th bead with respect to the moving frame, i.e.,

$$
{\underset{\sim}{v}}_{i}(t)={\underset{\sim}{\sim}}_{i}-\underset{\sim}{u}\left({\underset{\sim}{i}}_{i}(t), t\right), \quad i=1,2,
$$

where $\left.\underset{\sim}{u} \underset{\sim}{r_{i}}(t), t\right)$ is the solvent velocity at the point with position vector $\underset{\sim}{\underset{\sim}{r}}(t)$ at time $t$. Further, $B_{i}(t)$ denotes the $d$-component Brownian force acting on bead $i$ at time $t$, defined as the $d$-component Gaussian white noise multiplicatively scaled by $\sqrt{2 k \mu \zeta}$, i.e.,

$$
\left\langle\underset{\sim}{B_{i}}\right\rangle=\underset{\sim}{0}, \quad i=1,2, \quad \text { and } \quad\left\langle\underset{\sim}{B_{i}}(t)\left[\underset{\sim}{B_{j}}\left(t^{\prime}\right)\right]^{\top}\right\rangle=2 k \mu \zeta \delta_{i j} \delta\left(t-t^{\prime}\right) \underset{\sim}{I}, \quad i, j=1,2,
$$


where $\underset{\sim}{I}$ is the $d \times d$ identity matrix, and the angle brackets $\langle\cdot\rangle$ denote (in this section of the paper only) the ensemble average over the phase space $\left\{\left(\underset{\sim}{r_{1}}, \underset{\sim}{r_{2}},{\underset{\sim}{\sim}}_{1}, \dot{\sim}_{2}\right) \in \mathbb{R}^{4 d}\right\}$ of possible realizations. The elastic force $\underset{\sim}{F}: D \subseteq \mathbb{R}^{d} \rightarrow \mathbb{R}^{d}$ of the spring connecting the two beads is defined by a (sufficiently smooth) potential $U: \mathbb{R}_{\geq 0} \rightarrow \mathbb{R}$ through

$$
\underset{\sim}{F}(\underset{\sim}{q})=H U^{\prime}\left(\frac{1}{2}|\underset{\sim}{q}|^{2}\right) \underset{\sim}{q}
$$

where $H \in \mathbb{R}_{>0}$ is a spring constant. It follows from the definition of $\underset{\sim}{F}$ that

$$
\underset{\sim}{F}(\underset{\sim}{q})+\underset{\sim}{F}(-\underset{\sim}{q})=\underset{\sim}{0} \text { and } \underset{\sim}{F}(\underset{\sim}{q})-\underset{\sim}{F}(-\underset{\sim}{q})=2 \underset{\sim}{F}(\underset{\sim}{q}) \text {. }
$$

Example 1.1. Typical examples include the Hookean (linear) spring force $\underset{\sim}{F}(q)$ $=H \underset{\sim}{q}$, where $\underset{\sim}{q} \in D=\mathbb{R}^{d}$, corresponding to $U(s)=s$; and the finitely extensible nonlinear elastic (FENE) spring force

$$
\underset{\sim}{F}(\underset{\sim}{q})=\frac{H \underset{\sim}{q}}{1-|q|^{2} / Q_{\max }^{2}}, \quad \underset{\sim}{q} \in D=\left\{\underset{\sim}{q} \in \mathbb{R}^{d}:|\underset{\sim}{q}|<Q_{\max }\right\},
$$

corresponding to $U(s)=-\frac{1}{2} Q_{\max }^{2} \ln \left(1-\frac{2 s}{Q_{\max }^{2}}\right)$, where $Q_{\max }$ is the maximum length of the spring.

Neglecting the acceleration terms in Langevin's equation, as $m$ is small, the equations of motion of the beads become

$$
\begin{aligned}
& \zeta\left(\mathrm{d}{\underset{\sim}{r}}_{1}(t)-\underset{\sim}{u}\left({\underset{\sim}{1}}_{1}(t), t\right) \mathrm{d} t\right)=\underset{\sim}{B} 1(t) \mathrm{d} t+\underset{\sim}{F}\left({\underset{\sim}{r} 2}_{2} \underset{\sim}{r} 1\right) \mathrm{d} t, \\
& \zeta\left(\mathrm{d}{\underset{\sim}{\sim} 2}_{2}(t)-\underset{\sim}{u}\left({\underset{\sim}{2}}_{2}(t), t\right) \mathrm{d} t\right)=\underset{\sim}{B_{2}}(t) \mathrm{d} t+\underset{\sim}{F}(\underset{\sim}{r} 1-\underset{\sim}{r}) \mathrm{d} t .
\end{aligned}
$$

Let $f:\left({\underset{\sim}{1}}_{1},{\underset{\sim}{r}}_{2}, \dot{\sim}_{1}, \dot{\sim}_{2}, t\right) \mapsto f\left({\underset{\sim}{1}}_{1},{\underset{\sim}{2}}_{2}, \dot{\sim}_{1}, \dot{\sim}_{2}, t\right)$ denote the phase-space probability density function, defined as the nonnegative function $f$ such that $\int_{\mathcal{A}} f\left({\underset{\sim}{1}}_{1},{ }_{\sim} 2, \dot{\sim}_{\sim}, \dot{\sim}_{2}, t\right)$ $\mathrm{d}{ }_{\sim} \mathrm{d} r_{2} \mathrm{~d} \dot{\sim}_{1} \mathrm{~d} \dot{\sim}_{2}$ is the expected number of dumbbells at time $t$ having bead positions and velocities in the Borel set $\mathcal{A}$ of the phase space $\mathbb{R}^{4 d}$. We define the velocity-space average $\left\langle\langle A\rangle\right.$ of a function $A:\left(\underset{\sim}{r_{1}}, \underset{\sim}{2}, \dot{\sim}_{1}, \dot{\sim}_{2}\right) \mapsto A\left({\underset{\sim}{1}}_{1}, \underset{\sim}{r_{2}}, \dot{\sim}_{1}, \dot{\sim}_{\sim}\right)$ by

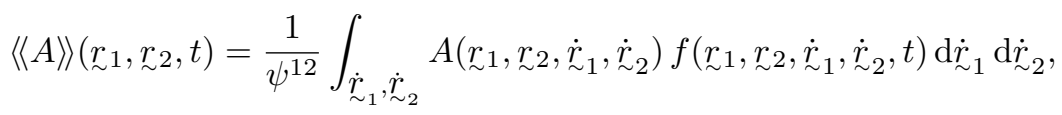

where the contracted configuration distribution function $\psi^{12}\left(\underset{\sim}{r_{1}}, \underset{\sim}{\sim}, t\right)$ is defined as the marginal distribution of $f$, that is,

$$
\psi^{12}\left(\underset{\sim}{r_{1}}, \underset{\sim}{r_{2}}, t\right)=\int_{\dot{\sim}_{\sim}, \dot{\sim}_{2}} f\left(\sim_{\sim} 1, \sim_{\sim},{\underset{\sim}{r}}_{1}, \dot{\sim}_{2}, t\right) \mathrm{d} \dot{\sim}_{\sim} \mathrm{d} \dot{\sim}_{\sim 2} .
$$

With these definitions, $\langle\langle 1\rangle\rangle=1$. By virtue of Liouville's theorem from statistical mechanics, $\psi^{12}$ satisfies the following continuity equation:

$$
\frac{\partial \psi^{12}}{\partial t}+\nabla_{r_{1}} \cdot\left(\left\langle\left\langle\dot{r}_{1}\right\rangle\right\rangle \psi^{12}\right)+\nabla_{r_{2}} \cdot\left(\left\langle\left\langle\dot{\sim}_{2}\right\rangle\right\rangle \psi^{12}\right)=0 .
$$

Applying the velocity-space average $\langle\langle\cdot\rangle\rangle$ to $(1.4 \mathrm{a}, \mathrm{b})$, we obtain

$$
\begin{aligned}
& \zeta\left(\left\langle\left\langle\dot{\sim}_{\sim}(t)\right\rangle\right\rangle-\underset{\sim}{u}\left({\underset{\sim}{r}}_{1}(t), t\right)\right)=\left\langle\left\langle\underset{\sim}{B} B_{1}(t)\right\rangle\right\rangle+\underset{\sim}{F}\left(\underset{\sim}{r_{2}}-{\underset{\sim}{r}}_{1}\right), \\
& \zeta\left(\left\langle\left\langle\dot{\sim}_{2}(t)\right\rangle\right\rangle-\underset{\sim}{u}\left(\underset{\sim}{r_{2}}(t), t\right)\right)=\left\langle\left\langle\underset{\sim}{B}{ }_{2}(t)\right\rangle\right\rangle+\underset{\sim}{F}\left(\sim_{\sim}^{r} 1-\underset{\sim}{r}\right) .
\end{aligned}
$$


Now, by adopting the Curtiss-Bird-Hassager hypothesis of equilibration in momentum space [7], the velocity-averaged Brownian force $\left\langle\left\langle\underset{\sim}{i}{ }_{i}\right\rangle\right\rangle, i=1,2$, satisfies

$$
\left\langle\left\langle{\underset{\sim}{i}}_{i}(t)\right\rangle=-k \mu \underset{\sim}{\nabla_{r_{i}}} \ln \psi^{12} .\right.
$$

We note in passing that Schieber and Öttinger highlight in [27] that equilibration in momentum space and neglecting the acceleration term in Langevin's equation are a consequence of a single hypothesis - that one can formally let the bead mass $m \rightarrow 0_{+}$.

Thus, (1.6a,b) yield

$$
\begin{aligned}
& \left\langle\left\langle\dot{\sim}_{1}\right\rangle\right\rangle \psi^{12}=-\frac{k \mu}{\zeta} \nabla_{r_{1}} \psi^{12}+\underset{\sim}{u}\left(\underset{\sim}{r_{1}}, t\right) \psi^{12}+\frac{1}{\zeta} \underset{\sim}{F}\left(\underset{\sim}{r_{2}}-\underset{\sim}{r_{1}}\right) \psi^{12}, \\
& \left\langle\left\langle\dot{\sim}_{2}\right\rangle\right\rangle \psi^{12}=-\frac{k \mu}{\zeta} \nabla_{r_{2}} \psi^{12}+\underset{\sim}{u}\left(\underset{\sim}{(}{ }_{2}, t\right) \psi^{12}+\frac{1}{\zeta} \underset{\sim}{F}\left(\underset{\sim}{\left.r_{1}-r_{2}\right)} \psi^{12} .\right.
\end{aligned}
$$

Substituting these into (1.5), we obtain the following Fokker-Planck equation for $\psi^{12}$ :

$$
\begin{aligned}
\frac{\partial \psi^{12}}{\partial t} & \left.+\underset{\sim}{\nabla} \nabla_{1} \cdot\left[\underset{\sim}{u} \underset{\sim}{r_{1}}, t\right) \psi^{12}+\frac{1}{\zeta} \underset{\sim}{F} \underset{\sim}{\left(r_{2}-\underset{\sim}{r_{1}}\right)} \psi^{12}\right] \\
& \left.+\underset{\sim}{\nabla} r_{r_{2}} \cdot\left[\underset{\sim}{u} \underset{\sim}{\left(r_{2}\right.}, t\right) \psi^{12}+\frac{1}{\zeta} \underset{\sim}{F} \underset{\sim}{\left(r_{1}-\underset{\sim}{r_{2}}\right)} \psi^{12}\right]=\frac{k \mu}{\zeta} \Delta_{r_{1}} \psi^{12}+\frac{k \mu}{\zeta} \Delta_{r_{2}} \psi^{12} .
\end{aligned}
$$

Recalling (1.3) and defining

$$
\psi(\underset{\sim}{x} \underset{\sim}{q}, t)=\psi^{12}\left(\underset{\sim}{x}-\frac{1}{2} \underset{\sim}{q}, \underset{\sim}{x}+\frac{1}{2} \underset{\sim}{q}, t\right),
$$

based on changing to center-of-mass coordinates, $\underset{\sim}{r}(t)=\frac{1}{2}\left(\underset{\sim}{r}(t)+{\underset{\sim}{r}}_{2}(t)\right)$, we deduce from (1.7) that

$$
\begin{aligned}
& \frac{\partial \psi}{\partial t}+\nabla_{x} \cdot\left(\frac{\underset{\sim}{u}\left(\underset{\sim}{x}-\frac{1}{2} \underset{\sim}{q}, t\right)+\underset{\sim}{u}\left(\underset{\sim}{x}+\frac{1}{2} \underset{\sim}{q}, t\right)}{2} \psi\right) \\
& \left.+\nabla_{q} \cdot\left(\left[\underset{\sim}{u}\left(\underset{\sim}{x}+\frac{1}{2} \underset{\sim}{q}, t\right)-\underset{\sim}{u}\left(\underset{\sim}{x}-\frac{1}{2} \underset{\sim}{q}, t\right)\right] \psi-\frac{2}{\zeta} \underset{\sim}{F} \underset{\sim}{q}\right) \psi\right)=\frac{k \mu}{2 \zeta} \Delta_{x} \psi+\frac{2 k \mu}{\zeta} \Delta_{q} \psi .
\end{aligned}
$$

In order to ensure that the definition of $\psi(\underset{\sim}{x}, \underset{\sim}{q}, t)$ is meaningful for all $\underset{\sim}{x} \in \Omega$, we shall suppose that $\underset{\sim}{q} \in D(\underset{\sim}{x})$, where

$$
D(x)=\left\{\underset{\sim}{q} \in D: \underset{\sim}{x} \pm s \underset{\sim}{q} \in \Omega \forall s \in\left[-\frac{1}{2}, \frac{1}{2}\right]\right\} .
$$

Hence, the set $D(\underset{\sim}{x})$ of admissible end-to-end vectors depends on the choice of $\underset{\sim}{x} \in \Omega$. Since $D$ has been assumed to be balanced, the same is true of $D(\underset{\sim}{x})$. Note, in particular, that since the macroscopic domain $\Omega$ is, by hypothesis, bounded, necessarily $|\underset{\sim}{q}| \leq \operatorname{diam}(\Omega)$ for any $\underset{\sim}{q} \in D(x)$.

Now, we can express

$$
\underset{\sim}{u}\left(\underset{\sim}{x}+\frac{1}{2} \underset{\sim}{q}, t\right)-\underset{\sim}{u}\left(\underset{\sim}{x}-\frac{1}{2} \underset{\sim}{q}, t\right)=\left({\underset{\sim}{x} x}_{\sim} \mathcal{J}_{1, q}^{x} u(\underset{\sim}{x}, t)\right) \underset{\sim}{q}, \quad \underset{\sim}{q} \in D(x),
$$


where $\mathcal{J}_{\alpha, q}^{x}: \underset{\sim}{w} \mapsto \mathcal{J}_{\alpha, q}^{x} \underset{\sim}{w}$ is the directional Friedrichs mollifier with respect to $\underset{\sim}{x}$, over an interval of length $\alpha|\underset{\sim}{ }|$, in the direction $\underset{\sim}{q}$, defined by

$$
\left(\mathcal{J}_{\alpha, q}^{x} \underset{\sim}{w}\right)(\underset{\sim}{x})=\frac{1}{\alpha} \int_{-\frac{\alpha}{2}}^{\frac{\alpha}{2}} \underset{\sim}{w}(\underset{\sim}{x}+\theta \underset{\sim}{q}) \mathrm{d} \theta, \quad \underset{\sim}{q} \in D(\underset{\sim}{x}), \quad \underset{\sim}{x} \in \Omega
$$

Thus, for $\underset{\sim}{q} \in D(\underset{\sim}{x})$ and $\underset{\sim}{x} \in \Omega$,

$$
\begin{aligned}
& \frac{\partial \psi}{\partial t}+\nabla_{x} \cdot\left(\frac{\underset{\sim}{u}\left(\underset{\sim}{x}-\frac{1}{2} \underset{\sim}{q}, t\right)+\underset{\sim}{u}\left(\underset{\sim}{x}+\frac{1}{2} \underset{\sim}{q}, t\right)}{2} \psi\right) \\
& +\nabla_{q} \cdot\left(\left(\underset{\approx}{\nabla_{x}} \mathcal{J}_{1, q}^{x} \underset{\sim}{u} \underset{\sim}{q} \psi-\underset{\zeta}{2} \underset{\sim}{F}(\underset{\sim}{q}) \psi\right)=\frac{k \mu}{2 \zeta} \Delta_{x} \psi+\frac{2 k \mu}{\zeta} \Delta_{q} \psi .\right.
\end{aligned}
$$

The fraction appearing in the second term on the left-hand side of this equation can be written as follows:

$$
\left.\frac{\underset{\sim}{u}\left(\underset{\sim}{x}-\frac{1}{2} \underset{\sim}{q}, t\right)+\underset{\sim}{u}\left(\underset{\sim}{x}+\frac{1}{2} \underset{\sim}{q}, t\right)}{2}=\underset{\sim}{u}(\underset{\sim}{x}, t)+\frac{1}{2} \Delta_{\frac{1}{2}}^{2} \underset{\sim}{\underset{\sim}{u}} \underset{\sim}{x}, t\right), \quad \underset{\sim}{q} \in D(\underset{\sim}{x}), \quad \underset{\sim}{x} \in \Omega,
$$

where

$$
\Delta_{\frac{1}{2}}^{2} \underset{\sim}{u} \underset{\sim}{u}(\underset{\sim}{x}, t)=\underset{\sim}{u}\left(\underset{\sim}{x}+\frac{1}{2} \underset{\sim}{q}, t\right)-2 \underset{\sim}{u}(\underset{\sim}{x}, t)+\underset{\sim}{u}\left(\underset{\sim}{x}-\frac{1}{2} \underset{\sim}{q}, t\right)
$$

is the second difference of $\underset{\sim}{u}$. Now, assuming that $\underset{\sim}{u}(\cdot, t)$ belongs to the Zygmund class $\mathcal{C}_{\text {loc }}^{1}$ (cf. [29], for example), we have that

$$
\left|\Delta_{\frac{1}{2}}^{2} \underset{\sim}{\sim} \underset{\sim}{u}(\underset{\sim}{x}, t)\right| \leq \frac{1}{2}|\underset{\sim}{q}||\underset{\sim}{u}(\cdot, t)|_{\mathcal{C}^{1}\left(B\left(\underset{\sim}{x}, \frac{1}{2}|q|\right)\right)}, \quad \underset{\sim}{q} \in D(\underset{\sim}{x}), \quad \underset{\sim}{x} \in \Omega,
$$

and hence

$$
\left|\frac{\underset{\sim}{u}\left(\underset{\sim}{x}-\frac{1}{2} \underset{\sim}{q}, t\right)+\underset{\sim}{u}\left(\underset{\sim}{x}+\frac{1}{2} q, t\right)}{2}-\underset{\sim}{u}(\underset{\sim}{x}, t)\right| \leq \frac{1}{4}|\underset{\sim}{q}||\underset{\sim}{u}(\cdot, t)|_{\mathcal{C}^{1}\left(B\left(\underset{\sim}{x}, \frac{1}{2}|q|\right)\right)}, \quad \underset{\sim}{q} \in D(\underset{\sim}{x}), \underset{\sim}{x} \in \Omega .
$$

The requirement $u \in \mathcal{C}_{\sim}^{1}$ loc is a very weak hypothesis on the regularity of $u$; in particular, $\underset{\sim}{u} \in \mathcal{C}_{\sim}^{1}$ loc may be nowhere differentiable on $\Omega$.

We proceed by adopting the local homogeneity assumption, $\ell_{0}|\underset{\sim}{u}(\cdot, t)|_{\mathcal{C}^{1}\left(B\left(x, \frac{1}{2} \ell_{0}\right)\right)}$ $\approx 0$, where $\ell_{0} \ll \operatorname{diam}(\Omega)$ is the characteristic microscopic length scale (of the characteristic dumbbell size). The validity of this assumption rests on the premise that, while the velocity field may exhibit wide variation with respect to $\underset{\sim}{x}$ over distances comparable to the size of an ensemble of dumbbells, its variation over the length scale $|q| \approx \ell_{0}$ of a single dumbbell is small. Under this hypothesis, the arithmetic mean $\frac{1}{2}\left(\underset{\sim}{u}\left(\underset{\sim}{x}-\frac{1}{2} q, t\right)+\underset{\sim}{u}\left(\underset{\sim}{x}+\frac{1}{2} q, t\right)\right)$ is simply replaced by $\underset{\sim}{u}(\underset{\sim}{x}, t)$. Hence, we arrive at the Fokker-Planck equation

$$
\frac{\partial \psi}{\partial t}+\underset{\sim}{\nabla_{x}} \cdot(\underset{\sim}{u}(\underset{\sim}{x}, t) \psi)+\underset{\sim}{\nabla_{q}} \cdot\left(\left({\underset{\sim}{\nabla}}_{x} \mathcal{J}_{1, q}^{x} \underset{\sim}{u}\right) \underset{\sim}{q} \psi-\frac{2}{\zeta} \underset{\sim}{F}(\underset{\sim}{q}) \psi\right)=\frac{k \mu}{2 \zeta} \Delta_{x} \psi+\frac{2 k \mu}{\zeta} \Delta_{q} \psi
$$

for $\underset{\sim}{x} \in \Omega, \underset{\sim}{q} \in D(\underset{\sim}{x}),|\underset{\sim}{q}|=\mathcal{O}\left(\ell_{0}\right)$, and $t>0$. The equation is supplemented by an initial condition $\psi(\underset{\sim}{\underset{\sim}{q}} \underset{\sim}{q}, 0)=\psi_{0}(\underset{\sim}{x} \underset{\sim}{q}) \geq 0$ and boundary conditions which will be specified later. 
Next we define the extra-stress tensor $\underset{\sim}{\tau}$ in terms of $\psi$. Taking an arbitrary surface in the dumbbell solution, we consider the contribution to $\underset{\approx}{\tau}$ at a point $P$ with position vector $\underset{\sim}{x}$ due to (a) the spring tension in the dumbbells $\widetilde{\widetilde{s}}$ traddling the surface at $P$; and (b) changes in momentum brought about by beads passing through the surface at $P$. Following Biller and Petruccione [4] and Petruccione and Biller [24], we then have that

$$
\begin{aligned}
\underset{\sim}{\mathcal{\tau}}(\underset{\sim}{x}, t) & =-2 k \mu \rho^{+}(\underset{\sim}{x}, t) \underset{\approx}{I}+\int_{D(\underset{\sim}{x})} \int_{-\frac{1}{2}}^{\frac{1}{2}} \underset{\sim}{q}[\underset{\sim}{F}(\underset{\sim}{q})]^{\top} \psi(\underset{\sim}{x}+\theta \underset{\sim}{q}, \underset{\sim}{q}, t) \mathrm{d} \theta \mathrm{d} \underset{\sim}{q} \\
& =-2 k \mu \rho^{+}(\underset{\sim}{x}, t) \underset{\approx}{I}+\int_{D(\underset{\sim}{x})} \underset{\sim}{q}[\underset{\sim}{F}(\underset{\sim}{q})]^{\top} \mathcal{J}_{1, q}^{x} \psi(\underset{\sim}{x}, \underset{\sim}{q}, t) \mathrm{d} \underset{\sim}{q},
\end{aligned}
$$

where $\mathcal{J}_{\alpha, q}^{x}$ is the scalar version of the operator $\mathcal{J}_{\alpha, q}^{x}$, and

$$
\rho^{+}(\underset{\sim}{x}, t)=\int_{D(\underset{\sim}{x})} \psi\left(\underset{\sim}{x}+\frac{1}{2} \underset{\sim}{q}, \underset{\sim}{q}, t\right) \mathrm{d} \underset{\sim}{q}, \quad \underset{\sim}{q} \in D(\underset{\sim}{x}), \quad|\underset{\sim}{q}|=\mathcal{O}\left(\ell_{0}\right), \quad \underset{\sim}{x} \in \Omega .
$$

We nondimensionalize $\underset{\sim}{q}$ by performing the change of variables $\underset{\sim}{\widehat{q}}=\underset{\sim}{q} / \ell_{0}$. Noting that

$$
\left(\mathcal{J}_{1, q}^{x} \underset{\sim}{w}\right)(\underset{\sim}{x})=\left(\mathcal{J}_{\sim}^{x} \ell_{0}, \widehat{q} \underset{\sim}{w}\right)(\underset{\sim}{x})=\frac{1}{\ell_{0}} \int_{-\frac{\ell_{0}}{2}}^{\frac{\ell_{0}}{2}} \underset{\sim}{w}(\underset{\sim}{x}+\theta \underset{\sim}{\widehat{q}}) \mathrm{d} \theta, \quad \underset{\sim}{q}=\ell_{0} \underset{\sim}{\widehat{q}} \in D(\underset{\sim}{x}), \quad \underset{\sim}{x} \in \Omega,
$$

the Fokker-Planck equation (1.9) becomes

$$
\frac{\partial \widehat{\psi}}{\partial t}+\nabla_{x} \cdot(\underset{\sim}{u} \widehat{\psi})+\nabla_{\widehat{q}} \cdot\left(\left({\underset{\sim}{\nabla}}_{x} \underset{\sim}{\mathcal{J}_{\ell_{0}}^{x} \widehat{q}} \underset{\sim}{u} \underset{\sim}{\widehat{q}} \widehat{\sim}-\frac{1}{2 \lambda} \underset{\sim}{\widehat{F}}(\underset{\sim}{\widehat{q}}) \widehat{\psi}\right)=\frac{\ell_{0}^{2}}{8 \lambda} \Delta_{x} \widehat{\psi}+\frac{1}{2 \lambda} \Delta_{\widehat{q}} \widehat{\psi},\right.
$$

where $\widehat{\psi}(\underset{\sim}{x}, \underset{\sim}{\widehat{q}}, t)=\ell_{0}^{d} \psi(\underset{\sim}{x}, \underset{\sim}{q}, t), \underset{\sim}{q}=\ell_{0} \underset{\sim}{\widehat{q}}, \ell_{0}=\sqrt{k \mu / H}, \lambda=\zeta / 4 H$, and

$$
\widehat{\sim}(\underset{\sim}{\widehat{q}})=\widehat{U}^{\prime}\left(\frac{1}{2}|\underset{\sim}{\widehat{q}}|^{2}\right) \underset{\sim}{\widehat{q}} \quad \text { with } \quad \widehat{U}(s)=\ell_{0}^{-2} U\left(\ell_{0}^{2} s\right) .
$$

We define $\widehat{D}=\ell_{0}^{-1} D$ and $\widehat{D}(\underset{\sim}{x})=\ell_{0}^{-1} D(\underset{\sim}{x})$.

Example 1.2. For the Hookean spring force, $\underset{\sim}{\widehat{F}}(\underset{\sim}{\widehat{q}})=\underset{\sim}{\widehat{q}}$ with $\underset{\sim}{\widehat{q}} \in \widehat{D}=\mathbb{R}^{d}$, corresponding to $\widehat{U}(s)=s$. For the FENE spring force,

$$
\underset{\sim}{\widehat{F}}(\underset{\sim}{\widehat{q}})=\frac{1}{1-|\underset{\sim}{\widehat{q}}|^{2} / b} \underset{\sim}{\widehat{q}}, \quad \underset{\sim}{q} \in \widehat{D}=\left\{\underset{\sim}{q} \in \mathbb{R}^{d}:|\underset{\sim}{\widehat{q}}|^{2}<b\right\},
$$

where $b=Q_{\max }^{2} / \ell_{0}^{2}$, corresponding to $\widehat{U}(s)=-\frac{b}{2} \ln \left(1-\frac{2 s}{b}\right),|s|<\frac{b}{2}$.

Changing variables in the expression for $\rho^{+}$, we deduce that

$$
\rho^{+}(\underset{\sim}{x}, t)=\int_{\widehat{D}(\underset{\sim}{x})} \widehat{\psi}\left(\underset{\sim}{x}+\frac{\ell_{0}}{2} \underset{\sim}{\widehat{q}}, \underset{\sim}{\widehat{q}}, t\right) \mathrm{d} \underset{\sim}{\widehat{q}} .
$$

By an identical argument, noting that $H \ell_{0}^{2}=k \mu$,

$$
\begin{aligned}
\underset{\sim}{\tau}(\underset{\sim}{x}, t) & =-2 k \mu \rho^{+}(\underset{\sim}{x}, t) \underset{\sim}{I}+H \ell_{0}^{2} \int_{\widehat{D}(\underset{\sim}{x})} \underset{\sim}{\widehat{q}} \widehat{\sim}^{\top} \widehat{U}^{\prime}\left(\frac{1}{2}|\underset{\sim}{\widehat{q}}|^{2}\right) \mathcal{J}_{\ell_{0}, \widehat{q}}^{x} \widehat{\psi}(\underset{\sim}{x}, \underset{\sim}{\widehat{q}}, t) \mathrm{d} \underset{\sim}{\widehat{q}} \\
& =p_{0}(\underset{\sim}{x}, t) \underset{\sim}{I}+k \mu\left(\int_{\widehat{D}(\underset{\sim}{x})} \underset{\sim}{\widehat{q}} \widehat{\sim}_{\sim}^{\top} \widehat{U}^{\prime}\left(\frac{1}{2}|\underset{\sim}{\widehat{q}}|^{2}\right) \mathcal{J}_{\ell_{0}, \widehat{q}}^{x} \widehat{\psi}(\underset{\sim}{x}, \underset{\sim}{\widehat{q}}, t) \mathrm{d} \underset{\sim}{\widehat{q}}-\rho(\underset{\sim}{x}, t) \underset{\approx}{I}\right),
\end{aligned}
$$


where

$$
p_{0}(\underset{\sim}{x}, t)=k \mu\left(\rho(\underset{\sim}{x}, t)-2 \rho^{+}(\underset{\sim}{x}, t)\right) \quad \text { and } \quad \rho(\underset{\sim}{x}, t)=\int_{\widehat{D}(\underset{\sim}{x})} \widehat{\psi}(\underset{\sim}{x}, \underset{\sim}{\widehat{q}}, t) \mathrm{d} \underset{\sim}{\widehat{q}} .
$$

Since the right-hand side of (1.1a) is equal to

$$
\begin{aligned}
\underset{\sim}{\nabla_{x}} \cdot \underset{\sim}{\tau}(x, t)= & \underset{\sim}{\nabla_{x}} p_{0}(\underset{\sim}{x}, t) \\
& +\underset{\sim}{\nabla_{x}} \cdot k \mu\left(\int_{\widehat{D}(\underset{\sim}{x})} \underset{\sim}{\widehat{q}} \widehat{q}^{\top} \widehat{U}^{\prime}\left(\underset{\sim}{\frac{1}{2}|\widehat{q}|^{2}}\right) \mathcal{J}_{\ell_{0}, \widehat{q}}^{x} \underset{\sim}{\widehat{\psi}}(\underset{\sim}{\widehat{q}}, t) \mathrm{d} \underset{\sim}{\widehat{q}}-\rho(\underset{\sim}{x, t)} \underset{\approx}{I}),\right.
\end{aligned}
$$

after transferring $\underset{\sim}{\nabla_{x}} p_{0}(\underset{\sim}{x}, t)$ to the left-hand side of (1.1a) and redefining the pressure $p(\underset{\sim}{x}, t)$ as $p(\underset{\sim}{x}, t)-p_{0}(\underset{\sim}{x}, t)$, the extra-stress tensor $\underset{\sim}{\tau}(\underset{\sim}{x}, t)$ becomes

$$
\underset{\sim}{\tau}(\underset{\sim}{x}, t)=k \mu\left(\int_{\widehat{D}(\underset{\sim}{x})} \underset{\sim}{\widehat{q}}{\underset{\sim}{q}}^{\top} \widehat{U}^{\prime}\left(\frac{1}{2}|\underset{\sim}{\widehat{q}}|^{2}\right) \mathcal{J}_{\ell_{0}, \widehat{q}}^{x} \widehat{\psi}(\underset{\sim}{x}, \underset{\sim}{\widehat{q}}, t) \mathrm{d} \underset{\sim}{\widehat{q}}-\rho(\underset{\sim}{x}, t) \underset{\sim}{I}\right),
$$

which is the Kramers expression for the elastic extra-stress tensor $\underset{\widetilde{\tau}}{\tau}$, except that here, in the case of a general heterogeneous solvent-velocity field $\underset{\sim}{u}(\underset{\sim}{x}, \widetilde{t})$, we have the directional Friedrichs mollification $\mathcal{J}_{\ell_{0}, \widehat{q}}^{x} \widehat{\psi}$ of $\widehat{\psi}$ instead of $\widehat{\psi}$ itself appearing in the classical Kramers expression.

We shall adopt the simplifying assumption that the configuration space $\widehat{D}$ is homogeneous, that is, $\widehat{D}(\underset{\sim}{x})=\widehat{D}$ for all $\underset{\sim}{x} \in \Omega$, where $\widehat{D}$ is a fixed balanced set, independent of $x$ (see, however, the papers [4, 24] cited above). For the sake of simplicity of exposition, we drop the 's from our notation: in what follows we shall write $\psi, \underset{\sim}{q}, \underset{\sim}{F}, U, D$ instead of $\underset{\psi}{\widehat{q}} \underset{\sim}{\widehat{T}} \underset{\sim}{\widehat{F}}, \widehat{U}, \widehat{D}$ throughout.

Thus, the governing equations become $(1.1 \mathrm{a}-\mathrm{d})$, where $\underset{\sim}{\tau}$ is defined by

$$
\underset{\sim}{\tau}(\underset{\sim}{x}, t)=k \mu\left(\int_{D} \underset{\sim}{q}{\underset{\sim}{\top}}^{\top} U^{\prime}\left(\frac{1}{2}|\underset{\sim}{q}|^{2}\right) \mathcal{J}_{\ell_{0}, q}^{x} \psi(\underset{\sim}{x} \underset{\sim}{q}, t) \mathrm{d} \underset{\sim}{q}-\rho(\underset{\sim}{x}, t) \underset{\sim}{I}\right),
$$

with

$$
\rho(\underset{\sim}{x}, t)=\int_{D} \psi(\underset{\sim}{x} \underset{\sim}{q}, t) \mathrm{d} q
$$

and, defining $\varepsilon=\ell_{0}^{2} / 8 \lambda$, we see that $\psi(\underset{\sim}{x} \underset{\sim}{q}, t)$ is a solution to the Fokker-Planck equation

$$
\frac{\partial \psi}{\partial t}+\left(\underset{\sim}{u} \cdot \nabla_{x}\right) \psi+\underset{\sim}{\nabla_{q}} \cdot\left(\left(\nabla_{\approx} \mathcal{J}_{\ell_{0}, q}^{x} \underset{\sim}{u} \underset{\sim}{q} \psi\right)=\varepsilon \Delta_{x} \psi+\frac{1}{2 \lambda} \nabla_{q} \cdot\left(\nabla_{q} \psi+U^{\prime} \underset{\sim}{q} \psi\right) .\right.
$$

This model has two noteworthy features compared to classical Fokker-Planck equations for bead-spring models appearing in the literature. The first of these is the presence of the $\underset{\sim}{x}$-dissipative center-of-mass diffusion term $\varepsilon \Delta_{x} \psi \equiv\left(\ell_{0}^{2} / 8 \lambda\right) \Delta_{x} \psi$ on the right-hand side of the Fokker-Planck equation (1.13). In standard derivations of bead-spring models the center-of-mass diffusion term is routinely omitted, on the grounds that it is several orders of magnitude smaller than the other terms in the equation. Indeed, when $L \approx 1$ is a characteristic macroscopic length scale 
(such as, for example, $\operatorname{diam}(\Omega)$ ), Bhave, Armstrong and Brown [3] estimate the ratio $\ell_{0}^{2} / L^{2}$ to be in the range of about $10^{-9}$ to $10^{-7}$. However, the omission of the term $\varepsilon \Delta_{x} \psi$ from (1.13) in the case of a heterogeneous solvent velocity $\underset{\sim}{u}(x, t)$ is a mathematically counterproductive model reduction. When $\varepsilon \Delta_{x} \psi$ is absent, (1.13) becomes a degenerate parabolic equation exhibiting hyperbolic behavior with respect to $(\underset{\sim}{x}, t)$. Since the study of weak solutions to the coupled problem requires one to work with velocity fields $u$ that have very limited Sobolev regularity (typically $\left.\underset{\sim}{u} \in L^{\infty}\left(0, T ; \sim_{\sim}^{2}(\Omega)\right) \cap L^{2}\left(0, T ; \underset{\sim}{H}{ }_{0}^{1}(\Omega)\right)\right)$, one is then forced into the technically unpleasant framework of hyperbolically degenerate parabolic equations with rough transport coefficients [1]. The resulting difficulties are further exacerbated by the fact that, when $D$ is bounded, a typical spring force $\underset{\sim}{F}(q)$ for a finitely extensible model (such as FENE) explodes as $q$ approaches $\partial D$; see Example 1.2 above. For these reasons, here we shall retain the center-of-mass diffusion term in (1.13). In fact, one of the objectives of this paper is to give mathematical foundation to the model reduction $\varepsilon=0$ by rigorously justifying the limiting process $\varepsilon \rightarrow 0_{+}$.

The second noteworthy feature of the model is the presence of the directional Friedrichs mollifier $\mathcal{J}_{\ell_{0}, q}^{x}$ in the Kramers expression (1.11) and in the Fokker-Planck equation (1.13). In standard derivations of these, upon postulating that $\psi$ and $\underset{\sim}{u}$ are sufficiently smooth, the local homogeneity assumption is used (in its classical form, expressed as the requirement that $u$ is "approximately linear" on the characteristic microscopic length scale $\left.\ell_{0}\right)$ to approximate $\mathcal{J}_{\ell_{0}, q}^{x} \psi(\underset{\sim}{x}, \underset{\sim}{q}, t)$ by $\psi(\underset{\sim}{x}, \underset{\sim}{q}, t)$ and $\mathcal{J}_{\ell_{0}, q}^{x} \underset{\sim}{u}(\underset{\sim}{x}, t)$ by $\underset{\sim}{u}(\underset{\sim}{x}, t)$ to simplify the model. We shall refrain from performing such approximations and will retain the Friedrichs mollifiers in the Kramers expression and in the Fokker-Planck equation, given that they naturally arise in the derivation of the model. Since the anisotropic mollifiers need to act in all possible directions $q$ contained in the balanced set $D$, we shall, instead, make a different, apparently morre reasonable, simplification which does not necessitate the imposition of additional smoothness requirements on $u$ or $\psi$ : we shall replace, in both (1.11) and (1.13), the directional Friedrichs mollifiers $\mathcal{J}_{\ell_{0}, q}^{x}$ and $\mathcal{J}_{\ell_{0}, q}^{x}$ by their isotropic counterparts $J_{\alpha}^{x}$ and $J_{\alpha}^{x}$, where $0<\alpha \leq \ell_{0}$. In the simplified case, when the drag term in (1.13) is corotational, that is, the tensor $\nabla_{x} u$ is replaced by its skew-symmetric part $\left.\frac{1}{2}\left(\left[\nabla_{x} u\right]-\left[\nabla_{x} u\right]^{\top}\right)\right)$, we shall rigorously justify, by passing to the limit $\alpha \rightarrow 0_{+}$, the model reduction $\alpha=0$ which corresponds to replacing the Friedrichs mollifiers by identity operators.

We conclude this introduction with a brief survey of developments on the analysis of classical bead-spring models, all of which correspond to formally letting $\varepsilon=0$ in (1.13), i.e., omitting the center-of-mass diffusion term, and formally letting $\alpha=0$, i.e., replacing the Friedrichs mollifiers $\mathcal{J}_{\alpha, q}^{x}$ and $\mathcal{J}_{\alpha, q}^{x}$ by identity operators.

An early effort to show the existence and uniqueness of local-in-time solutions to a family of bead-spring type polymeric flow models is due to Renardy [25]. While the class of potentials $\underset{\sim}{F}(\underset{\sim}{q})$ considered by Renardy [25] (cf. hypotheses $(\mathrm{F})$ and $\left(\mathrm{F}^{\prime}\right)$ on pp. 314-315) does include the case of Hookean dumbbells, it excludes the practically relevant case of the FENE model (see Example 1.2 above). More recently, E, Li, and Zhang [10] and Li, Zhang, and Zhang [17] have revisited the question of local existence of solutions for dumbbell models. A further development in this direction is the work of Zhang and Zhang [31], where the local existence of regular solutions to FENE-type models has been shown. All of these papers require high regularity of the initial data.

Constantin [6] has considered the Navier-Stokes equations coupled to nonlinear Fokker-Planck equations describing the evolution of the probability distribution of the particles interacting with the fluid. He described, in the case when $D$ is a Riemannian 
manifold, relations determining the coefficients of the stresses added in the fluid by the particles; these relations link the extra stresses to the kinematic effect of the fluid velocity on the particles and to the interparticle interaction potential. In equations (of Type 1, in the terminology of [6]) where the extra stresses depend linearly on the particle distribution density, as is the case in the present paper, the energy balance requires a response potential. In equations (of Type 2) where the added stresses depend quadratically on the particle distribution, it is shown that energy balance can be achieved without a dynamic response potential, and global existence of smooth solutions is shown if inertial effects are neglected. The necessary relationship (eq. (2.14) in [6]) for the existence of a Lyapunov function in the sense of Theorem 2.2 of [6] does not hold for the polymer models considered in the present paper.

Otto and Tzavaras [23] have investigated the Doi model (which is similar to a Hookean model considered here, except that $D=S^{2}$ ) for suspensions of rod-like molecules in the dilute regime. For certain parameter values, the velocity gradient vs. stress relation defined by the stationary and homogeneous flow is not rank-one monotone. They considered the evolution of possibly large perturbations of stationary flows and proved that, even in the absence of a microscopic cutoff, discontinuities in the velocity gradient cannot occur in finite time.

In a recent paper Jourdain, Lelièvre, and Le Bris [15] studied the existence of solutions to the FENE model in the case of a simple Couette flow; by using tools from the theory of stochastic differential equations, they established the existence of a unique local-in-time solution to the FENE model in two space dimensions $(d=2)$ when the velocity field $\underset{\sim}{u}$ is unidirectional and of the particular form $\underset{\sim}{u}\left(x_{1}, x_{2}\right)=$ $\left(u_{1}\left(x_{2}\right), 0\right)^{\top}$. The notion of solution for which existence is proved in the paper of Jourdain, Lelièvre, and Le Bris [15] is mixed deterministic-stochastic in the sense that it is deterministic in the "macroscopic" variable $\underset{\sim}{x}$ but stochastic in the "microscopic" variable $q$. In contrast, our notion of solution (cf. section 3 below) is deterministic both macroscopically and microscopically, since the microscales are modelled here by the probability density function $\psi(\underset{\sim}{x}, q, t)$. The choice between these different notions of solution has far-reaching consequences on computational simulation: mixed deterministic-stochastic notions of solution necessitate the use of Monte Carlo-type algorithms for the numerical approximation of polymer configurations, as proposed in the monograph of Öttinger [22] and, for example, in the paper of Jourdain, Lelièvre, and Le Bris [14]; whereas weak solutions in the sense considered in the present paper can be approximated by entirely deterministic (e.g., Galerkin-type) schemes, as was done, for example, in Lozinski et al. [20] — at the cost of solving a Fokker-Planck equation in $2 d$ spatial dimensions.

In the case of Hookean dumbbells, and assuming $\varepsilon=0$ and $\alpha=0$, the coupled microscopic-macroscopic model described above yields, formally, taking the second moment of $\underset{\sim}{q} \mapsto \psi(\underset{\sim}{q}, \underset{\sim}{x}, t)$, the fully macroscopic, Oldroyd-B model of viscoelastic flow (cf. section 2.2 below). Lions and Masmoudi [18] have shown the existence of globalin-time weak solutions to the Oldroyd-B model in a simplified corotational setting, as described in section 2.2 , but with $\alpha=0, \varepsilon=0, \sigma(\cdot)=\nabla_{x}(\cdot)$ on the right-hand side of (2.19), and $\underset{\widetilde{f}}{\sigma}(\cdot)=\underset{\widetilde{*}}{\omega}(\cdot)$ in the definition of the upper-convective derivative (2.17). The argument of Lions and Masmoudi [18] is based on exploiting the propagation in time of the compactness of the solution and the DiPerna-Lions theory [8] of renormalized solutions to linear hyperbolic equations with nonsmooth transport coefficients. It is not known if an identical global existence result for the Oldroyd-B model also holds in the absence of the crucial assumption that the drag term is corotational. We note in 
passing that, assuming $\varepsilon>0$ and $\alpha=0$, the coupled microscopic-maroscopic model above yields, taking the appropriate moments in the case of Hookean dumbbells, a dissipative version of the Oldroyd-B model. In this sense, the Hookean dumbbell model has a macroscopic closure: it is the Oldroyd-B model when $\varepsilon=0$, and a dissipative version of Oldroyd-B (cf. (2.19) below) when $\varepsilon>0$. In contrast, the FENE model is not known to have an exact closure at the macroscopic level, though $\mathrm{Du}, \mathrm{Yu}$, and Liu [9] and $\mathrm{Yu}, \mathrm{Du}$, and Liu [30] have recently considered the analysis of approximate closures of the FENE model. Previously, El-Kareh and Leal [11] had proposed a macroscopic model, with added dissipation in the equation which governs the evolution of the conformation tensor $\underset{\sim}{A}(\underset{\sim}{x}, t):=\int_{D} q q^{\top} \psi(\underset{\sim}{x}, \underset{\sim}{q}, t) \mathrm{d} q$ in order to account for Brownian motion across streamlines; the model can be thought of as an approximate macroscopic closure of a FENE-type micro-macro model with center-ofmass diffusion.

Simultaneously, Barrett, Schwab, and Süli [2] established the existence of globalin-time weak solutions to the coupled microscopic-macroscopic model (1.1a-d) and (1.13) with $\varepsilon=0$, an $x$-mollified velocity gradient in the Fokker-Planck equation and an $\underset{\sim}{x}$-mollified probability density function $\psi$ in the Kramers expression-admitting a large class of potentials $U$ (including the Hookean dumbbell model as well as general FENE-type models); in addition to these mollifications, $\underset{\sim}{u}$ in the $\underset{\sim}{x}$-convective term $(\underset{\sim}{u}$. $\left.\nabla_{x}\right) \psi$ in the Fokker-Planck equation was also mollified. Unlike Lions and Masmoudi [18], the arguments in [2] did not require the assumption that the drag term was corotational in the FENE case. The mollification $S_{\alpha} u$ of the velocity field $u$ that was considered in [2] was stimulated by the Leray- $\alpha$ model of the incompressible NavierStokes equations (the viscous Camassa-Holm equations), proposed by Foias, Holm, and Titi [12], and was defined as follows: the mollified velocity field $S_{\alpha} \underset{\sim}{u}=\underset{\sim}{v}$ is the solution of the following Helmholtz-Stokes problem:

$$
\underset{\sim}{v}-\alpha \Delta_{x} \underset{\sim}{v}+\underset{\sim}{\nabla} \pi=\underset{\sim}{u} \quad \text { in } \Omega, \quad{\underset{\sim}{x}}_{x} \cdot \underset{\sim}{v}=0 \quad \text { in } \Omega, \quad \underset{\sim}{v}=\underset{\sim}{0} \quad \text { on } \partial \Omega,
$$

where $\pi$ is a pressure-like auxiliary variable (with no particular physical meaning). This definition ensures that the mollified velocity field $S_{\alpha} \underset{\sim}{u}=\underset{\sim}{v}$ remains divergencefree and satisfies the same boundary condition as $\underset{\sim}{u}$. In [2] the motivation for introducing the mollification was of purely technical nature: the need to rigorously justify the passage to the limit in the proof of the existence of weak solutions, based on a compactness argument. It is interesting to observe on reflection that, when starting from first principles, the derivation of the coupled Navier-Stokes-Fokker-Planck model does, in fact, include a mollification of $\psi$ in the Kramers formula for the extrastress tensor as well as of the velocity gradient in the Fokker-Planck equation, just as in [2], albeit the mollifiers are directional Friedrichs mollifiers rather than HelmholtzStokes mollifiers. In classical derivations of the model, the mollifiers are approximated by identity operators, on the grounds that the functions to which they are applied are smooth enough to justify such a model reduction; absurdly, in the proof of existence of weak solutions to the reduced model, the mollifiers then have to be reinstated since the requisite smoothness hypotheses which were used to justify the model reduction are absent. Thus, in this paper we chose to retain the Friedrichs mollifiers which naturally arise in the derivation of the model - our only modelling approximation being to replace the directional Friedrichs mollifiers by their isotropic counterparts $J_{\alpha}^{x}$ and $J_{\alpha}^{x}, 0<\alpha \leq \ell_{0}$; in particular, unlike the argument presented in [2], here we do not mollify the $\underset{\sim}{x}$-convective term $\left(\underset{\sim}{u} \cdot \nabla_{x}\right) \psi$ in the Fokker-Planck equation. For the same reason, instead of formally neglecting the center-of-mass diffusion term $\varepsilon \Delta_{x} \psi$ in 
the Fokker-Planck equation (1.13) on the grounds that $\varepsilon \ll 1$, we consciously retain this term in our model, at least initially. We shall then rigorously justify the model reduction $\varepsilon=0$.

Our first objective is to show the existence of weak solutions to the complete model, corresponding to $\alpha>0$ and $\varepsilon>0$. This is accomplished in section 3 after formulating carefully in section 2 the class of potentials $U$ considered. In particular, we show in section 2 how the Hookean dumbbell model and the FENE model fit into the general setting. In addition, we introduce a family of weighted Sobolev spaces which represent the natural functional-analytic framework for the problem; we also recall from [2] some crucial density and trace results in these weighted spaces. These weighted space are closely related to the Fokker-Planck equation under consideration: the weight of the space is the Maxwellian induced by the potential $U$ appearing in the Fokker-Planck equation. We then rigorously justify the model reduction $\varepsilon=$ 0 . In particular, we rigorously pass to the limit $\varepsilon \rightarrow 0_{+}$in both the corotational and the general noncorotational case; in the latter case we confine ourselves to the physically relevant situation when $D$ is a bounded domain. We also justify the model reduction $\alpha=0$ in the corotational case by rigorously passing to the limit $\alpha \rightarrow$ $0_{+}$. The rigorous justification of the model reduction $\alpha=0$ remains open in the general noncorotational case; under our weak smoothness requirements on the data, the justification of the simultaneous model reduction $(\alpha, \varepsilon)=(0,0)$ also remains open. While our "macroscopic" energy estimate, which bounds the velocity field $\underset{\sim}{u}$ in terms of the data, is completely uniform with respect to both $\varepsilon$ and $\alpha$ in both the corotational and the general noncorotation case, the resulting compactness results are, unfortunately, of insufficient strength to admit rigorous passage to the simultaneous limit $(\alpha, \varepsilon) \rightarrow\left(0_{+}, 0_{+}\right)$.

2. Polymer models. We term polymer models under consideration here microscopic-macroscopic-type models, since the continuum mechanical macroscopic equations of incompressible fluid flow are coupled to a microscopic model: the FokkerPlanck equation describing the statistical properties of particles in the continuum. We first present these equations and collect assumptions on the parameters in the model.

2.1. Microscopic-macroscopic polymer models. Let $\Omega \subset \mathbb{R}^{d}$ be a bounded open set with a Lipschitz-continuous boundary $\partial \Omega$, and suppose that the set $D \subseteq \mathbb{R}^{d}$, $d=2$ or 3 , of admissible elongation vectors $q$ in (1.13) is an open set which may be bounded or unbounded. For the sake of simplicity of presentation, we shall suppose that $D$ is either a bounded open ball in $\mathbb{R}^{d}$, or $D=\mathbb{R}^{d}$; these two cases cover all practically relevant scenarios involving the microscopic-macroscopic models discussed here. Our arguments in the case when the configuration domain $D$ is a bounded open ball can be extended, with only minimal changes, to situations when $D$ is any bounded open domain in $\mathbb{R}^{d}$ with smooth boundary (e.g., an ellipse, to account for anisotropy in the molecule's configuration).

Our system of equations involves the following Friedrichs mollifier with respect to $\underset{\sim}{x}$. Let $j \in W^{1, \infty}\left(\mathbb{R}^{d}\right)$ with compact support in the closed unit ball $B(0,1)$, such that $\int_{B(\underset{\sim}{0}, 1)} j(\underset{\sim}{x}) \mathrm{d} \underset{\sim}{x}=1, j(\underset{\sim}{x}) \geq 0$, and $j(-\underset{\sim}{x})=j(\underset{\sim}{x})$ for all $\underset{\sim}{x} \in B(\underset{\sim}{0}, 1)$. Then for any $\alpha \in(0,1]$, let $\left(J_{\alpha}^{x} \eta\right)(\underset{\sim}{x}): L^{1}(\Omega) \rightarrow W^{1, \infty}(\Omega)$ be such that

$$
\left(J_{\alpha}^{x} \eta\right)(\underset{\sim}{x})=\int_{\Omega} j_{\alpha}(\underset{\sim}{x}-\underset{\sim}{y}) \eta(\underset{\sim}{y}) \mathrm{d} y \quad \forall \underset{\sim}{x} \in \Omega
$$


where $\left.j_{\alpha} \underset{\sim}{x}\right)=\alpha^{-d} j\left(\alpha^{-1} \underset{\sim}{x}\right)$. In addition, we extend $J_{\alpha}^{x}$ in the natural way to vector and tensor functions to obtain $\underset{\sim}{J}: \underset{\sim}{L^{1}}(\Omega) \rightarrow \underset{\sim}{W^{1, \infty}}(\Omega)$ and $\underset{\sim}{\underset{\sim}{x}}: \underset{\sim}{L^{1}}(\Omega) \rightarrow \underset{\sim}{W^{1, \infty}}(\Omega)$.

Gathering (1.1a-d), (1.11), and (1.13) together, we then consider the following initial-boundary-value problem dependent on parameters $\alpha, \varepsilon \in(0,1]$ :

$\left(\mathrm{P}_{\alpha, \varepsilon}\right)$ Find ${\underset{\sim}{\alpha, \varepsilon}}_{\alpha}:(\underset{\sim}{x}, t) \in \mathbb{R}^{d+1} \mapsto u_{\alpha, \varepsilon}(\underset{\sim}{x}, t) \in \mathbb{R}^{d}$ and $p_{\alpha, \varepsilon}:(\underset{\sim}{x}, t) \in \mathbb{R}^{d+1} \mapsto$ $p_{\alpha, \varepsilon}(\underset{\sim}{x}, t) \in \mathbb{R}$ such that

(2.2a) $\frac{\partial u_{\alpha, \varepsilon}}{\partial t}+\left(u_{\alpha, \varepsilon} \cdot \nabla_{x}\right) u_{\alpha, \varepsilon}-\nu \Delta_{x} u_{\alpha, \varepsilon}+\nabla_{x} p_{\alpha, \varepsilon}=\nabla_{x} \cdot{\underset{\sim}{\tau}}^{(\alpha)}\left(\psi_{\alpha, \varepsilon}\right)$

$$
\begin{aligned}
& \text { in } \Omega \times(0, T] \text {, } \\
& \nabla_{x} \cdot{\underset{\sim}{u} \alpha, \varepsilon}_{\alpha}=0 \quad \text { in } \Omega \times(0, T], \\
& \sim_{\alpha, \varepsilon}=\underset{\sim}{0} \quad \text { on } \partial \Omega \times(0, T], \\
& {\underset{\sim}{\alpha}, \varepsilon}_{\alpha}(\underset{\sim}{x}, 0)={\underset{\sim}{u}}_{0}(\underset{\sim}{x}) \quad \forall \underset{\sim}{x} \in \Omega,
\end{aligned}
$$

where $\nu \in \mathbb{R}_{>0}$ is the viscosity and $\underset{\sim}{\tau^{(\alpha)}}\left(\psi_{\alpha, \varepsilon}\right):(\underset{\sim}{x}, t) \in \mathbb{R}^{d+1} \mapsto \underset{\widetilde{\tau}}{\tau^{(\alpha)}}\left(\psi_{\alpha, \varepsilon}\right)(\underset{\sim}{x}, t) \in$ $\mathbb{R}^{d \times d}$ is the symmetric extra-stress tensor, dependent on a probability density function $\psi_{\alpha, \varepsilon}:(\underset{\sim}{x}, \underset{\sim}{q}, t) \in \mathbb{R}^{2 d+1} \mapsto \psi_{\alpha, \varepsilon}(\underset{\sim}{x}, \underset{\sim}{q}, t) \in \mathbb{R}$, defined as

$$
\underset{\approx}{\tau^{(\alpha)}}\left(\psi_{\alpha, \varepsilon}\right)=k \mu\left(\underset{\approx}{C}\left(J_{\alpha}^{x} \psi_{\alpha, \varepsilon}\right)-\rho\left(\psi_{\alpha, \varepsilon}\right) \underset{\approx}{I}\right) \equiv k \mu\left(\underset{\approx}{J}\left[\underset{\approx}{C}\left(\psi_{\alpha, \varepsilon}\right)\right]-\rho\left(\psi_{\alpha, \varepsilon}\right) \underset{\approx}{I}\right) .
$$

Here $k, \mu \in \mathbb{R}_{>0}$ are, respectively, the Boltzmann constant and the absolute temperature, $\underset{\approx}{I}$ is the unit $d \times d$ tensor, and

$$
\underset{\sim}{C}\left(\psi_{\alpha, \varepsilon}\right)(\underset{\sim}{x}, t)=\int_{D} \psi_{\alpha, \varepsilon}(\underset{\sim}{x} \underset{\sim}{q}, t) U^{\prime}\left(\frac{1}{2}|\underset{\sim}{q}|^{2}\right) \underset{\sim}{q}{\underset{\sim}{\top}}^{\top} \mathrm{d} \underset{\sim}{q}
$$

and

$$
\rho\left(\psi_{\alpha, \varepsilon}\right)(\underset{\sim}{x}, t)=\int_{D} \psi_{\alpha, \varepsilon}(\underset{\sim}{x}, \underset{\sim}{q}, t) \mathrm{d} q \underset{\sim}{ } .
$$

In addition, the real-valued, continuous, nonnegative, and strictly monotonic increasing function $U$, defined on a relatively open subset of $[0, \infty)$, is an elastic potential which gives the elastic force $\underset{\sim}{F}: D \rightarrow \mathbb{R}^{d}$ on the springs via (1.2).

Roughly speaking, the probability density $\psi_{\alpha, \varepsilon}(\underset{\sim}{x}, \underset{\sim}{q}, t)$ represents the probability at time $t$ of finding the center of mass of a dumbbell at $\underset{\sim}{x}$ and having elongation vector $\underset{\sim}{q}$. Hence $\left.\rho\left(\psi_{\alpha, \varepsilon}\right) \underset{\sim}{x}, t\right)$ is the density of the polymer chains located at $\underset{\sim}{x}$ at time $t$. It follows from (1.13) that $\psi_{\alpha, \varepsilon}$ satisfies the Fokker-Planck equation

$$
\begin{aligned}
& \frac{\partial \psi_{\alpha, \varepsilon}}{\partial t}+(\underset{\sim}{u, \varepsilon} \cdot \underset{\sim}{\nabla}) \psi_{\alpha, \varepsilon}+\underset{\sim}{\nabla} \cdot\left(\underset{\sim}{\sigma}\left({\underset{\sim}{\alpha}}_{\alpha}^{x} \underset{\alpha, \varepsilon}{ }\right) \underset{\sim}{q} \psi_{\alpha, \varepsilon}\right) \\
& =\frac{1}{2 \lambda} \nabla_{q} \cdot\left(\nabla_{q} \psi_{\alpha, \varepsilon}+U^{\prime} \underset{\sim}{q} \psi_{\alpha, \varepsilon}\right)+\varepsilon \Delta_{x} \psi_{\alpha, \varepsilon} \quad \text { in } \Omega \times D \times(0, T],
\end{aligned}
$$

together with suitable initial and boundary conditions which will be stated below.

In (2.5) the parameter $\lambda \in \mathbb{R}_{>0}$ characterizes the elastic relaxation property of

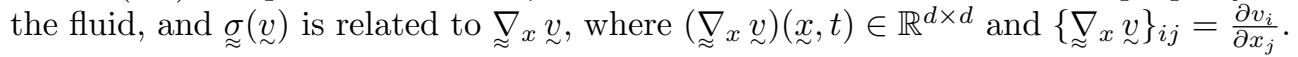
We will be interested in two possible choices:

(i) the noncorotational case $\underset{\sim}{\sigma}(\underset{\sim}{v})=\underset{\sim}{\underset{\sim}{v}} \underset{\sim}{v}$ 
or

(ii) the corotational case $\underset{\approx}{\sigma} \underset{\sim}{v})=\underset{\approx}{\omega}(\underset{\sim}{v})$,

where

$$
\begin{aligned}
& \left.\nabla_{x} v=\underset{\sim}{v}=\underset{\sim}{D(v)}+\underset{\sim}{\omega(v)}, \quad \underset{\sim}{D}(\underset{\sim}{v})=\frac{1}{2} \underset{\approx}{\nabla_{x}} \underset{\sim}{v}+\left(\underset{\approx}{\nabla_{x} v}\right)^{\top}\right], \\
\text { and } \quad & \left.\underset{\sim}{\omega}(\underset{\sim}{v})=\frac{1}{2} \underset{\approx}{\nabla_{x}} \underset{\sim}{v}-\left(\underset{\approx}{\nabla_{x}} \underset{\sim}{v}\right)^{\top}\right] .
\end{aligned}
$$

In the corotational case no smoothing is necessary in the extra-stress tensor, and so we will replace $(2.3)$ by

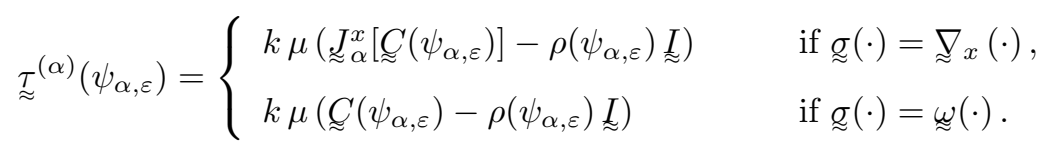

Introducing the (normalized) Maxwellian

$$
M(\underset{\sim}{q})=\frac{\mathrm{e}^{-U\left(\frac{1}{2}\left|\underline{\sim}^{q}\right|^{2}\right)}}{\int_{D} \mathrm{e}^{-U} \mathrm{~d} \underset{\sim}{q}},
$$

we have that

$$
M \underset{\sim}{\underset{q}{\nabla}} M^{-1}=-M^{-1} \underset{\sim}{\nabla_{q}} M=U^{\prime} \underset{\sim}{q} .
$$

In addition, the following identities hold:

$$
\nabla_{q} U=U^{\prime} \underset{\sim}{q}, \quad \nabla_{q} U^{\prime}=U^{\prime \prime} \underset{\sim}{q}, \quad \text { and } \quad \Delta_{q} U=U^{\prime \prime}|\underset{\sim}{q}|^{2}+U^{\prime} d .
$$

Thus, the Fokker-Planck equation (2.5) can be rewritten as

$$
\begin{aligned}
& \frac{\partial \psi_{\alpha, \varepsilon}}{\partial t}+\left({\underset{\sim}{u} \alpha, \varepsilon}_{\sim} \cdot \nabla_{x}\right) \psi_{\alpha, \varepsilon}+\nabla_{q} \cdot\left(\underset{\approx}{\sigma}\left(J_{\alpha}^{x} u_{\alpha, \varepsilon}\right) \underset{\sim}{q} \psi_{\alpha, \varepsilon}\right) \\
& =\frac{1}{2 \lambda} \nabla_{q} \cdot\left(M \underset{\sim}{\nabla_{q}}\left(\frac{\psi_{\alpha, \varepsilon}}{M}\right)\right)+\varepsilon \Delta_{x} \psi_{\alpha, \varepsilon} \quad \text { in } \Omega \times D \times(0, T] .
\end{aligned}
$$

We impose the following boundary and initial conditions:

$$
\begin{aligned}
\psi_{\alpha, \varepsilon}=0 & \text { on } \Omega \times \partial D \times(0, T], \\
\varepsilon \nabla_{x} \psi_{\alpha, \varepsilon} \cdot \underset{\sim}{n}=0 & \text { on } \partial \Omega \times D \times(0, T], \\
\psi_{\alpha, \varepsilon}(\underset{\sim}{x}, \underset{\sim}{q}, 0)=\psi_{0}(\underset{\sim}{x}, \underset{\sim}{q}) \geq 0 & \forall(\underset{\sim}{x}, \underset{\sim}{q}) \in \Omega \times D,
\end{aligned}
$$

where $\underset{\sim}{n}$ is normal to $\partial \Omega$. Here $\int_{\Omega \times D} \psi_{0}(\underset{\sim}{x}, \underset{\sim}{q}) \mathrm{d} \underset{\sim}{q} \mathrm{~d} \underset{\sim}{x}=1$. The boundary conditions for $\psi_{\alpha, \varepsilon}$ on $\partial \Omega \times D \times(0, T]$ and $\Omega \times \partial D \times(0, T]$ have been chosen so as to ensure that $\int_{\Omega \times D} \psi_{\alpha, \varepsilon}(\underset{\sim}{x}, \underset{\sim}{q}, t) \mathrm{d} \underset{\sim}{q} \mathrm{~d} \underset{\sim}{x}=1$ for all $t \geq 0$. As we shall see later, the choice of the function space for $\psi_{\alpha, \varepsilon}$ and the decay properties of the Maxwellian near $\partial D$ under the structural conditions stated in section 2.2 below imply that both $\psi_{\alpha, \varepsilon}=0$ and $\nabla_{q} \psi_{\alpha, \varepsilon} \cdot \underset{q}{q}=0$ on $\Omega \times \partial D \times(0, T]$. Hence, the boundary condition $(2.12 \mathrm{a})$ will be seen to be redundant.

When $D=\mathbb{R}^{d}$, the boundary condition (2.12a) on $\partial D$, the boundary of $D$, is replaced by a decay condition at infinity which demands that $|\psi|$ converges to 0 sufficiently fast as $|\underset{\sim}{q}|$ tends to $\infty$; we shall be more specific about this later. 


\subsection{Two examples.}

2.2.1. FENE-type models. A widely used model is the FENE model, where $D=B\left(\underset{\sim}{0}, b^{\frac{1}{2}}\right)$ and

$$
U(s)=-\frac{b}{2} \ln \left(1-\frac{2 s}{b}\right), \quad \text { and hence } \quad \mathrm{e}^{-U\left(\frac{1}{2}|q|^{2}\right)}=\left(1-\frac{\left.|q| q\right|^{2}}{b}\right)^{\frac{b}{2}} .
$$

Here $B(\underset{\sim}{0}, s)$ is the bounded open ball of radius $s>0$ in $\mathbb{R}^{d}$ centered at the origin, and $b>0$ is an input parameter. Hence the length $|\underset{\sim}{\mid}|$ of the elongation vector $\underset{\sim}{q}$ cannot exceed $b^{\frac{1}{2}}$.

2.2.2. Hookean dumbbells. Letting $b \rightarrow \infty$ in (2.13) leads to the so-called Hookean dumbbell model, where

$$
D=\mathbb{R}^{d} \quad \text { and } \quad U(s)=s, \quad \text { and therefore } \quad \mathrm{e}^{-U\left(\frac{1}{2}|q|^{2}\right)}=\mathrm{e}^{-\frac{1}{2}|q|^{2}} .
$$

This particular kinetic model for $\varepsilon, \alpha \in(0,1]$, with $\underset{\sim}{\sigma}\left(\sim_{\alpha}^{x} u_{\alpha, \varepsilon}\right)=\underset{\sim}{\nabla}\left({\underset{\sim}{\alpha}}_{\alpha}^{x} u_{\alpha, \varepsilon}\right)$, corresponds formally to an Oldroyd-B type model, or with $\underset{\sim}{\sigma}\left(J_{\alpha}^{x} u_{\alpha, \varepsilon}\right)=\underset{\sim}{\omega}\left(J_{\alpha}^{x} u_{\alpha, \varepsilon}\right)$ to a corotational Oldroyd-B type model. Indeed, multiplying (2.5) by $\underset{\sim}{q} q^{\top}$, integrating over $D$, performing integration by parts (assuming that $\psi_{\alpha, \varepsilon}$ and $\left|\widetilde{\nabla}_{q} \psi_{\alpha, \varepsilon}\right|$ decay to zero sufficiently fast as $|\underset{\sim}{q}| \rightarrow \infty$ ), and noting (2.4a) and for any $\underset{\sim}{\underset{\sim}{\sim}} \in \mathbb{R}^{d}$ that

$$
\left(\underset{\sim}{r} \cdot \sim_{q}\right) \underset{\sim}{q} \sim_{\sim}^{\top}=\underset{\sim}{r}{\underset{\sim}{q}}^{\top}+\underset{\sim}{q}{\underset{\sim}{r}}^{\top} \quad \text { and } \quad \Delta_{q}\left(\underset{\sim}{q}{\underset{\sim}{q}}^{\top}\right)=2 \underset{\sim}{I}
$$

yields that $\underset{\approx}{C} \alpha, \varepsilon(\underset{\sim}{x}, t) \equiv \underset{\sim}{C}\left(\psi_{\alpha, \varepsilon}(\underset{\sim}{x}, t)\right)$ satisfies

$$
\lambda\left(\frac{\delta \mathcal{\approx}_{\alpha, \varepsilon}}{\delta t}-\varepsilon \Delta_{x} \underset{\approx \alpha, \varepsilon}{C_{\alpha, \varepsilon}}\right)+\underset{\approx}{C_{\alpha, \varepsilon}}=\rho_{\alpha, \varepsilon} \underset{\approx}{I} \quad \text { in } \Omega \times(0, T],
$$

where $\rho_{\alpha, \varepsilon}(\underset{\sim}{x}, t) \equiv \rho\left(\psi_{\alpha, \varepsilon}(\underset{\sim}{x}, t)\right)$ and

$$
\frac{\delta \underset{\approx}{C}}{\delta t}=\frac{\partial \underset{\approx}{C}}{\partial t}+\left(u_{\alpha, \varepsilon} \cdot \underset{\sim}{\nabla}\right) \underset{\approx}{C}-\left[\underset{\approx}{\sigma}\left(J_{\alpha}^{x} u_{\alpha, \varepsilon}\right) \underset{\approx}{C}+\underset{\approx}{C}\left[\underset{\sim}{\sigma}\left(J_{\alpha}^{x} u_{\alpha, \varepsilon}\right)\right]^{\top}\right]
$$

is the upper-convected time derivative. Similarly, integrating (2.5) over $D$ and noting (2.4b) yields that $\rho_{\alpha, \varepsilon}$ satisfies

$$
\frac{\partial \rho_{\alpha, \varepsilon}}{\partial t}-\varepsilon \Delta_{x} \rho_{\alpha, \varepsilon}+\left(\underset{\sim}{u} \cdot \underset{\sim}{\nabla_{x}}\right) \rho_{\alpha, \varepsilon}=0 \quad \text { in } \Omega \times(0, T] .
$$

Hence in the Hookean case, the probability density function $\psi_{\alpha, \varepsilon}$ can be eliminated, leading to a closed model for $u_{\alpha, \varepsilon}, C_{\alpha, \varepsilon}$, and $\rho_{\alpha, \varepsilon}$. Moreover, if either $\alpha=0$ or $\underset{\sim}{\sigma(v)}) \underset{\sim}{\omega}(\underset{\sim}{v})$, then $(2.16)$ and $(2.18)$ can be combined, noting (2.8), to yield that the extra stress $\underset{\sim}{\tau} \alpha, \varepsilon(\underset{\sim}{x}, t) \equiv \underset{\sim}{\tau}(\alpha)\left(\psi_{\alpha, \varepsilon}\right)$ satisfies

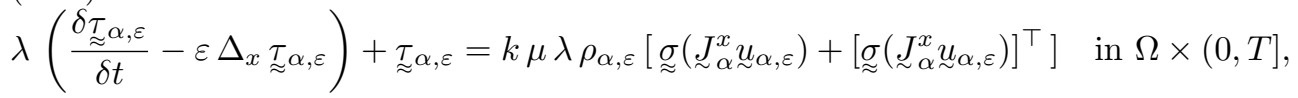

which, in the case of formally setting $\varepsilon=\alpha=0$, is the Oldroyd-B constitutive equation if $\underset{\sim}{\sigma}(\underset{\sim}{u})=\underset{\approx}{\nabla_{x}} \underset{\sim}{u}$ or the corotational Oldroyd-B constitutive equation if $\underset{\sim}{\sigma}(u)=\underset{\sim}{\omega}(u)$; in the latter case, the right-hand side of (2.19) is identically equal to 0 . 
2.3. General structural assumptions on the potential. Suppose that $D$ is a bounded open ball in $\mathbb{R}^{d}$ or $D=\mathbb{R}^{d}$. We assume that $\underset{\sim}{q} \mapsto U\left(\frac{1}{2}|q|^{2}\right) \in C^{\infty}(D)$ with $\underset{\sim}{q} \mapsto U\left(\frac{1}{2}|\underset{\sim}{\mid}|^{2}\right)$ nonnegative and $\underset{\sim}{q} \mapsto U^{\prime}\left(\frac{1}{2}|q|^{2}\right)$ positive on $D$, and that there exist constants $\tilde{c}_{i}>0, i=1,2$, such that

$$
\left(U^{\prime}\right)^{2}-U^{\prime \prime} \geq c_{1} \quad \forall \underset{\sim}{q} \in D \quad \text { and } \quad\left(U^{\prime}\right)^{2}-U^{\prime \prime} \geq 2 c_{2} U^{\prime} \quad \forall \underset{\sim}{q}: \quad|\underset{\sim}{q}|^{2} \geq \frac{d}{c_{2}},
$$

where $B\left(\underset{\sim}{0},\left(\frac{d}{c_{2}}\right)^{\frac{1}{2}}\right) \subset \subset D$.

The above assumptions hold for the Hookean case, (2.14), with $c_{1}=2 c_{2}=1$; and the FENE case, (2.13), assuming that $b>2$, with $c_{1}=\frac{b-2}{b}$ and $c_{2}=\frac{b+2 d-2}{2 b}$.

We shall also suppose that there exist positive constants $c_{i}, i=3, \ldots, 7$, and $\kappa>0$, such that the Maxwellian $M$ and the associated elastic potential $U$ satisfy

(2.21a) $c_{3}[\operatorname{dist}(\underset{\sim}{q}, \partial D)]^{\kappa} \leq M(\underset{\sim}{q}) \leq c_{4}[\operatorname{dist}(\underset{\sim}{q}, \partial D)]^{\kappa} \quad \forall \underset{\sim}{q} \in D$

$(2.21 \mathrm{~b}) c_{5} \leq[\operatorname{dist}(\underset{\sim}{q}, \partial D)] U^{\prime}\left(\frac{1}{2}\left|\sim_{\sim}^{q}\right|^{2}\right) \leq c_{6}, \quad[\operatorname{dist}(\underset{\sim}{q}, \partial D)]^{2}\left|U^{\prime \prime}\left(\frac{1}{2} \mid{\underset{\sim}{q}}^{2}\right)\right| \leq c_{7} \quad \forall \underset{\sim}{ } \in D ;$

when $D=\mathbb{R}^{d}$, then $[\operatorname{dist}(q, \partial D)]^{\kappa}$ in $(2.21 \mathrm{a})$ is replaced by $\exp \left(-|q|^{2}\right)$, and the factors $[\operatorname{dist}(q, \partial D)]$ and $[\operatorname{dist}(q, \partial D)]^{2}$ in $(2.21 \mathrm{~b})$ are omitted.

It is an easy matter to show that the Maxwellian $M$ and the elastic potential $U$ of the FENE model and of the Hookean dumbbell model satisfy conditions $(2.21 \mathrm{a}, \mathrm{b})$ with $D=B\left(\underset{\sim}{0}, b^{\frac{1}{2}}\right)$ and $\kappa=\frac{b}{2}$ in the case of the FENE model; and $D=\mathbb{R}^{d}$ for the Hookean dumbbell model.

We shall also require that

$$
\int_{D}\left[1+\left(1+|\underset{\sim}{q}|^{2}\right)\left((U)^{2}+|\underset{\sim}{q}|^{2}\left(U^{\prime}\right)^{2}\right)\right] M \mathrm{~d} \underset{\sim}{q}<\infty .
$$

For the Hookean model (2.14) and the FENE model (2.13), with $b>2,(2.22)$ is easily shown to hold. For example, we have that

$$
\mathcal{M}:=\int_{D} M\left(U^{\prime}\right)^{2}|\underset{\sim}{q}|^{4} \mathrm{~d} \underset{\sim}{q}<\infty
$$

for both models. In the Hookean case, (2.23) follows since

$$
\int_{0}^{\infty} \mathrm{e}^{-s} s^{\frac{d+2}{2}} \mathrm{~d} s<\infty
$$

while in the FENE case, (2.23) follows since

$$
\int_{0}^{b}\left(1-\frac{s}{b}\right)^{\frac{b-4}{2}} s^{\frac{d+2}{2}} \mathrm{~d} s<\infty \quad \text { if } b>2 .
$$

More generally, it follows from $(2.21 \mathrm{a}, \mathrm{b})$, noting that $U\left(\underset{2}{2}|\underset{\sim}{\sim}|^{2}\right)=-\ln M(\underset{\sim}{q})+$ Const., that (2.22) holds, provided that either (i) $\kappa>1$ when $\tilde{D}$ is a bounded open ball in $\mathbb{R}^{d}$; or (ii) when $D=\mathbb{R}^{d}$.

\section{Existence of global weak solutions. Let}

$$
\underset{\sim}{H}:=\left\{\underset{\sim}{w} \in \underset{\sim}{L^{2}}(\Omega): \underset{\sim}{\nabla} \cdot \underset{\sim}{w}=0\right\} \quad \text { and } \quad \underset{\sim}{V}:=\{\underset{\sim}{w} \in \underset{\sim}{H}(\Omega): \underset{\sim}{\nabla} \cdot \underset{\sim}{w}=0\},
$$


where the divergence operator $\nabla_{x}$. is to be understood in the sense of vector-valued

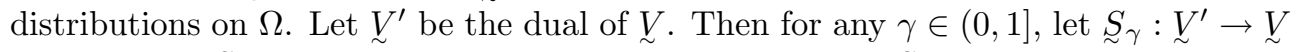
be such that ${\underset{\sim}{\gamma}}_{\gamma} \underset{\sim}{v}$ is the unique solution to the Helmholtz-Stokes problem

$$
\int_{\Omega} \underset{\sim}{S_{\gamma}} \underset{\sim}{v} \cdot \underset{\sim}{w} \mathrm{~d} \underset{\sim}{x}+\gamma \int_{\Omega} \underset{\sim}{\nabla} \underset{\sim}{S_{\gamma}} \underset{\sim}{v}: \underset{\sim}{\nabla} \underset{\sim}{w} \mathrm{~d} \underset{\sim}{x}=\langle\underset{\sim}{v}, \underset{\sim}{w}\rangle \quad \forall \underset{\sim}{w} \in \underset{\sim}{V},
$$

where $\langle\cdot, \cdot\rangle$ denotes the duality pairing between $\underset{\sim}{V^{\prime}}$ and $\underset{\sim}{V}$. We note that

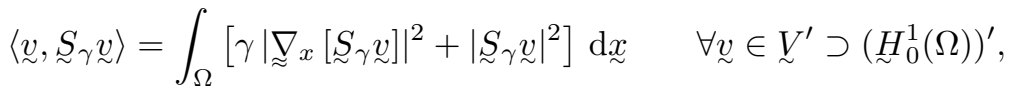

and $\left\|S_{\gamma} \cdot\right\|_{H^{1}(\Omega)}$ is a norm on $V^{\prime}$. In addition, we have from (3.2) that

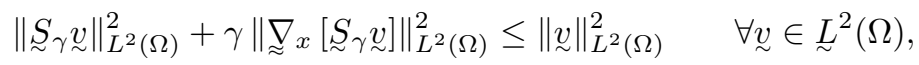

$$
\begin{aligned}
& \left\|\left(\underset{\sim}{I}-{\underset{\sim}{\gamma}}_{\gamma}\right) \underset{\sim}{v}\right\|_{L^{2}(\Omega)}^{2}+\gamma\left\|\nabla_{x}(\underset{\sim}{I}-\underset{\sim}{S}) \underset{\sim}{v}\right\|_{L^{2}(\Omega)}^{2}
\end{aligned}
$$

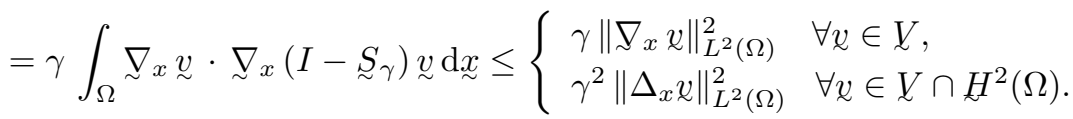

Hence it follows from $(3.4 \mathrm{~b})$ that

$$
\left\|\left(\underset{\sim}{I}-\underset{\sim}{S_{\gamma}}\right) \underset{\sim}{v}\right\|_{H^{1}(\Omega)} \leq \gamma^{\frac{1}{2}}\left\|\Delta_{x} \underset{\sim}{v}\right\|_{L^{2}(\Omega)} \leq(\gamma d)^{\frac{1}{2}}\|\underset{\sim}{v}\|_{H^{2}(\Omega)} \quad \forall \underset{\sim}{\mathcal{v}} \in \underset{\sim}{V} \cap \underset{\sim}{H^{2}}(\Omega) .
$$

Furthermore, for $\partial \Omega \in C^{2}$ and $r>d$ (cf. Girault and Raviart [13, p. 88]) we have that

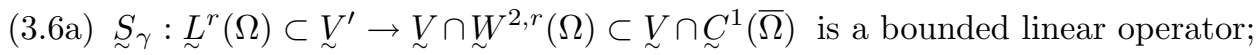
and hence Sobolev embedding yields that

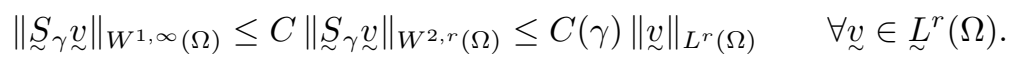

The aims of this paper are to prove existence of a (global-in-time) solution of a weak formulation of (i) the problem $\left(\mathrm{P}_{\alpha, \varepsilon}\right)$ for any fixed parameters $\alpha, \varepsilon \in(0,1]$; (ii) the problem $\left(\mathrm{P}_{\alpha}\right)$, obtained by formally setting $\varepsilon=0$ in $\left(\mathrm{P}_{\alpha, \varepsilon}\right)$ (cf. $\left.(3.93 \mathrm{a}, \mathrm{b})\right)$, for any fixed parameter $\alpha \in(0,1]$ for both the corotational and general noncorotational cases under the following assumptions on the data:

(3.7) $\partial \Omega \in C^{0,1}, \quad \underset{\sim}{u_{0}} \in \underset{\sim}{H}, \quad$ and $\quad M^{-\frac{1}{2}} \psi_{0} \in L^{2}(\Omega \times D)$ with $\psi_{0} \geq 0$ a.e. in $\Omega \times D$.

In addition, if $\partial \Omega \in C^{2}$ we prove existence of a (global-in-time) solution of a weak formulation of the problem $\left(\mathrm{P}_{\varepsilon}\right)$, obtained by formally setting $\alpha=0$ in $\left(\mathrm{P}_{\alpha, \varepsilon}\right)$ (cf. $(3.100 \mathrm{a}, \mathrm{b}))$, for any fixed parameter $\varepsilon \in(0,1]$, in the corotational case only.

The following results for $J_{\alpha}^{x}$ are easily established:

$$
\begin{aligned}
\left\|J_{\alpha}^{x} \eta\right\|_{L^{2}(\Omega)} & \leq\|\eta\|_{L^{2}(\Omega)} & & \forall \eta \in L^{2}(\Omega), \\
\left\|\left(I-J_{\alpha}^{x}\right) \eta\right\|_{L^{2}(\Omega)} & \rightarrow 0 \quad \text { as } \alpha \rightarrow 0 & & \forall \eta \in L^{2}(\Omega), \\
\int_{\Omega}\left(J_{\alpha}^{x} \eta_{1}\right) \eta_{2} \mathrm{~d} x & =\int_{\Omega} \eta_{1}\left(J_{\alpha}^{x} \eta_{2}\right) \mathrm{d} x & & \forall \eta_{1}, \eta_{2} \in L^{2}(\Omega), \\
\frac{\partial}{\partial x_{i}}\left(J_{\alpha}^{x} \eta\right) & =J_{\alpha}^{x}\left(\frac{\partial \eta}{\partial x_{i}}\right), \quad i=1 \rightarrow d & & \forall \eta \in H_{0}^{1}(\Omega) .
\end{aligned}
$$


It follows from (3.8a) and (3.8d) that $J_{\alpha}^{x}$ satisfies

$$
\begin{array}{cc}
\left\|\underset{\sim}{J_{\alpha}^{x} v \|_{H^{1}(\Omega)}} \leq\right\| \underset{\sim}{v} \|_{H^{1}(\Omega)} & \forall \underset{\sim}{v} \in \underset{\sim}{H_{0}^{1}(\Omega),} \\
\left\|J_{\sim}^{x} \underset{\sim}{v}\right\|_{W^{1, \infty}(\Omega)} \leq C(\alpha)\|\underset{\sim}{v}\|_{L^{1}(\Omega)} & \forall \underset{\sim}{v} \in \underset{\sim}{L^{1}(\Omega) .}
\end{array}
$$

We note that the results $(3.8 \mathrm{a}-\mathrm{d})$ and $(3.9 \mathrm{a})$ hold if $j \in L^{\infty}\left(\mathbb{R}^{d}\right)$, with compact support in the closed unit ball $B(\underset{\sim}{0}, 1), j(\underset{\sim}{x}) \geq 0$ for $\underset{\sim}{x} \in \mathbb{R}^{d}, \int_{B(\underset{0}{0}, 1)} j(\underset{\sim}{x}) \mathrm{d} x=1$, and $j(-\underset{\sim}{x})=j(\underset{\sim}{x})$ for all $\underset{\sim}{x} \in B(\underset{\sim}{0}, 1)$, e.g., a constant multiple of the characteristic function of $B(0,1)$. However, we require the result $(3.9 \mathrm{~b})$ for our existence proof, and hence our restriction to $j \in W^{1, \infty}\left(\mathbb{R}^{d}\right)$ instead of $j \in L^{\infty}\left(\mathbb{R}^{d}\right)$.

Introducing

$$
\|\varphi\|_{H^{0,1}(\Omega \times D ; M)}:=\left\{\int_{\Omega \times D} M\left[|\varphi|^{2}+\left|\nabla_{q} \varphi\right|^{2}\right] \mathrm{d} \underset{\sim}{q} \mathrm{~d} \underset{\sim}{ }\right\}^{\frac{1}{2}}
$$

and

$$
\|\varphi\|_{H^{1}(\Omega \times D ; M)}:=\left\{\int_{\Omega \times D} M\left[|\varphi|^{2}+\left|\nabla_{x} \varphi\right|^{2}+\left|\nabla_{q} \varphi\right|^{2}\right] \mathrm{d} \underset{\sim}{q} \mathrm{~d} x\right\}^{\frac{1}{2}}
$$

we then set

$$
H^{0,1}(\Omega \times D ; M):=\left\{\varphi \in L_{\mathrm{loc}}^{1}(\Omega \times D):\|\varphi\|_{H^{0,1}(\Omega \times D ; M)}<\infty\right\}
$$

and

$$
H^{1}(\Omega \times D ; M):=\left\{\varphi \in L_{\text {loc }}^{1}(\Omega \times D):\|\varphi\|_{H^{1}(\Omega \times D ; M)}<\infty\right\} .
$$

We then define

$$
\begin{aligned}
X & :=M H^{1}(\Omega \times D ; M), \\
X_{q} & :=\left\{\varphi \in X: \int_{\Omega \times D} \mid \underset{\sim}{|q|^{2}} \frac{|\varphi|^{2}}{M} \mathrm{~d} \underset{\sim}{q} \mathrm{~d} \underset{\sim}{x}<\infty\right\}, \\
X^{+} & :=\{\varphi \in X: \varphi(\underset{\sim}{x}, \underset{\sim}{q}) \geq 0 \text { for a.e. }(\underset{\sim}{x}, \underset{\sim}{q}) \in \Omega \times D\},
\end{aligned}
$$

and

$$
X_{q}^{+}:=X_{q} \cap X^{+} .
$$

Clearly, if $D$ is bounded, then $X_{q}=X$ and $X_{q}^{+}=X^{+}$. We remark, in particular, that due to the structural hypotheses on $U$ (specifically, (2.22) and (2.21a)), both $M$ and $M U$ belong to $X_{q}^{+}$. Similarly to above, we define $X^{0}, X_{q}^{0}, X^{0,+}$, and $X_{q}^{0,+}$, where $X^{0}:=M H^{0,1}(\Omega \times D ; M)$. 
We note for future reference that (2.4a) and (2.23) yield that, for $\varphi \in X^{0}$,

$$
\begin{aligned}
\int_{\Omega}|C(\varphi)|^{2} \mathrm{~d} x & =\int_{\Omega} \sum_{i=1}^{d} \sum_{j=1}^{d}\left(\int_{D} \varphi U^{\prime} q_{i} q_{j} \mathrm{~d} \underset{\sim}{\mathrm{d} q}\right)^{2} \mathrm{~d} \underset{\sim}{x} \\
& \leq d\left(\int_{D} M\left(U^{\prime}\right)^{2} \mid \underset{\sim}{|q|^{4}} \underset{\sim}{\mathrm{d} q}\right)\left(\int_{\Omega \times D} \frac{|\varphi|^{2}}{M} \underset{\sim}{\mathrm{d} q} \underset{\sim}{\mathrm{d} x}\right) \\
& =d \mathcal{M}\left(\int_{\Omega \times D} \frac{|\varphi|^{2}}{M} \underset{\sim}{\mathrm{d}} \underset{\sim}{\mathrm{d} x}\right) .
\end{aligned}
$$

Recalling Lemma 3.1 in Barrett, Schwab, and Süli [2], we have that

$$
\mathcal{K}^{0}:= \begin{cases}M \cdot C^{\infty}(\overline{\Omega \times D}) & \text { if } D \text { is a bounded open ball in } \mathbb{R}^{d} \\ C_{0}^{\infty}(\Omega \times D) & \text { if } D \equiv \mathbb{R}^{d}\end{cases}
$$

is dense in $X_{q}^{0}$. It is a simple matter to adapt the proof there to show that

$$
\mathcal{K}:= \begin{cases}M \cdot C^{\infty}(\overline{\Omega \times D}) & \text { if } D \text { is a bounded open ball in } \mathbb{R}^{d}, \\ C^{\infty}\left(\bar{\Omega} ; C_{0}^{\infty}(D)\right) & \text { if } D \equiv \mathbb{R}^{d}\end{cases}
$$

is dense in $X_{q}$. In addition, recalling Lemma 3.2 in Barrett, Schwab, and Süli [2], we have that

(a) if $D$ is a bounded open ball in $\mathbb{R}^{d}$ and the elastic potential $U$ and the associated Maxwellian $M$ satisfy (2.21a) with $\kappa \geq 5$ and (2.21b), then

$$
U^{\prime}\left(\frac{1}{2}|q|^{2}\right) \varphi=0 \quad \text { on } \Omega \times \partial D \quad \forall \varphi \in X^{0} ;
$$

(b) if $D=\mathbb{R}^{d}$, then, for all $\varphi \in X^{0}$,

$$
\lim _{R \rightarrow \infty} R^{\beta} \int_{\Omega \times \partial B(\underset{\sim}{0}, R)} U^{\prime}\left(\frac{1}{2}|\underset{\sim}{q}|^{2}\right)|\varphi| \mathrm{d} S(\underset{\sim}{q)} \mathrm{d} \underset{\sim}{x}=0 \quad \forall \beta \geq 0 .
$$

Recalling Lemma 3.3 in Barrett, Schwab, and Süli [2], we have for any constant $L \geq 0$ that

$$
\varphi \in X_{q}^{0} \quad \Rightarrow \quad[\varphi-L M]_{+},[\varphi+L M]_{-} \in X_{q}^{0} .
$$

Of course, this result remains true if $X_{q}^{0}$ is replaced by $X_{q}$.

We note that

$$
\underset{\sim}{\omega}(v)=-[\underset{\sim}{\omega}(v)]^{\top} \text { and hence } \underset{\sim}{q} \underset{\sim}{\top} \underset{\sim}{\omega}(\underset{\sim}{q}) \underset{\sim}{q}=0 \quad \forall \underset{\sim}{q} \in \mathbb{R}^{d} .
$$

Recalling (4.15a,b) in Barrett, Schwab, and Süli [2], it follows for all $\underset{\sim}{v} \in W^{1, \infty}(\Omega)$ that

$$
\begin{aligned}
\int_{\Omega \times D} \varphi(\underset{\sim}{(\underset{\sim}{v})} \underset{\sim}{q}) \cdot \nabla_{q}\left(\frac{\varphi}{M}\right) \mathrm{d} \underset{\sim}{q} \mathrm{~d} \underset{\sim}{x}=0, & \\
\int_{\Omega \times D} M(\underset{\sim}{(\underset{\sim}{v})} \underset{\sim}{q}) \cdot \nabla_{q}\left(\frac{\varphi}{M}\right) \mathrm{d} \underset{\sim}{q} \mathrm{~d} \underset{\sim}{x}=0 & \forall \varphi \in X_{q}^{0} .
\end{aligned}
$$


As no smoothing is required of the extra-stress tensor on the right-hand side of the Navier-Stokes equations in the corotational case, we introduce

$$
\underset{\sim}{I_{\alpha}}:=\left\{\begin{array}{lll}
J_{\alpha}^{x} & \text { if } & \underset{\sim}{ }(\cdot)=\bigotimes_{x}(\cdot), \\
L & \text { if } & g(\cdot)=\underset{\sim}{ }(\cdot) .
\end{array}\right.
$$

3.1. Existence for $\left(\mathbf{P}_{\alpha, \varepsilon}\right)$. In this subsection we will prove existence of a solution to the following weak formulation of $\left(\mathrm{P}_{\alpha, \varepsilon}\right)$ for given parameters $\alpha, \varepsilon \in(0,1]$ :

$\left(\mathrm{P}_{\alpha, \varepsilon}\right)$ Find ${\underset{\sim}{\alpha, \varepsilon}}_{\alpha} \in L^{\infty}\left(0, T ;{\underset{\sim}{L}}^{2}(\Omega)\right) \cap L^{2}(0, T ; \underset{\sim}{V}) \cap W^{1, \frac{4}{d}}\left(0, T ; V^{\prime}\right)$ and $\psi_{\alpha, \varepsilon} \in$ $L^{2}(0, T ; X)$, with $J_{\alpha}^{x} u_{\alpha, \varepsilon} \in L^{\infty}\left(0, T ; W^{1, \infty}(\Omega)\right), M^{-\frac{1}{2}} \psi_{\alpha, \varepsilon} \in L^{\infty}\left(0, T ; L^{2}(\Omega \times D)\right)$, and $\underset{\sim}{C}\left(\psi_{\alpha, \varepsilon}\right) \in L^{\infty}(0, T ; \underset{\sim}{L}(\Omega))$, such that $\underset{\sim}{\sim} \underset{\alpha, \varepsilon}{ }(\cdot, 0)={\underset{\sim}{u}}_{0}(\cdot)$ and

$$
\begin{aligned}
\int_{0}^{T}\left\langle\frac{\partial u_{\alpha, \varepsilon}}{\partial t}, \underset{\sim}{w}\right\rangle \mathrm{d} t & +\int_{0}^{T} \int_{\Omega}\left[\left[\left(u_{\alpha, \varepsilon} \cdot \nabla_{x}\right) u_{\alpha, \varepsilon}\right] \cdot \underset{\sim}{w}+\nu \underset{\sim}{\nabla_{x}} \underset{\sim}{u_{\alpha, \varepsilon}}:{\underset{\sim}{\nabla}}_{x} \underset{\sim}{w}\right] \mathrm{d} x \underset{\sim}{\mathrm{d} t} \\
& =-k \mu \int_{0}^{T} \int_{\Omega} \underset{\approx}{C}\left(\psi_{\alpha, \varepsilon}\right):{\underset{\sim}{\nabla}}_{x}\left(\underset{\sim}{I_{\alpha}} \underset{\sim}{w}\right) \mathrm{d} \underset{\sim}{x} \mathrm{~d} t \quad \forall \underset{\sim}{w} \in L^{\frac{4}{4-d}}(0, T ; V),
\end{aligned}
$$

$$
\begin{aligned}
& -\int_{0}^{T} \int_{\Omega \times D} \frac{\psi_{\alpha, \varepsilon}}{M} \frac{\partial \varphi}{\partial t} \mathrm{~d} \underset{\sim}{q} \mathrm{~d} \underset{\sim}{\mathrm{d}} \mathrm{d} t-\int_{\Omega \times D} \frac{\psi_{0}(\cdot, \cdot)}{M} \varphi(\cdot, \cdot, 0) \mathrm{d} \underset{\sim}{\mathrm{d}} \mathrm{d} x \\
& +\int_{0}^{T} \int_{\Omega \times D}\left[\frac{M}{2 \lambda} \nabla_{q}\left(\frac{\psi_{\alpha, \varepsilon}}{M}\right)-\left[\underset{\sim}{\sigma}\left(J_{\alpha}^{x} u_{\alpha, \varepsilon}\right) \underset{\sim}{q}\right] \psi_{\alpha, \varepsilon}\right] \cdot \nabla_{q}\left(\frac{\varphi}{M}\right) \mathrm{d} q \underset{\sim}{\mathrm{d}} \mathrm{d} \mathrm{d} t \\
& +\int_{0}^{T} \int_{\Omega \times D}\left[\varepsilon M{\underset{\sim}{\nabla} x}_{x}\left(\frac{\psi_{\alpha, \varepsilon}}{M}\right)-{\underset{\sim}{\alpha, \varepsilon}}_{\alpha} \psi_{\alpha, \varepsilon}\right] \cdot \nabla_{x}\left(\frac{\varphi}{M}\right) \mathrm{d} \underset{\sim}{\mathrm{d}} \mathrm{d} \underset{\sim}{x} \mathrm{~d} t=0 \quad \forall \varphi \in \mathcal{X},
\end{aligned}
$$

where $\mathcal{X}$ is the completion of $C_{0}^{\infty}((-T, T) ; \mathcal{K})$ in the norm $\|\cdot\|_{\mathcal{X}}$ defined by

$$
\begin{aligned}
\|\varphi\|_{\mathcal{X}}:=\|\varphi\|_{L^{\frac{4}{4-d}}\left(0, T ; X_{q}\right)}+\left\|\underset{\sim}{|q| M^{\frac{1}{2}} \underset{\sim}{\nabla_{q}}\left(\frac{\varphi}{M}\right)}\right\|_{L^{1}\left(0, T ; L^{2}(\Omega \times D)\right)} \\
+\left\|M^{-\frac{1}{2}} \frac{\partial \varphi}{\partial t}\right\|_{L^{1}\left(0 ; T ; L^{2}(\Omega \times D)\right)} .
\end{aligned}
$$

This, in particular, implies that each $\varphi \in \mathcal{X}$ satisfies $\varphi(\cdot, \cdot, T)=0$.

Remark 3.1. If $d=2$, then ${\underset{\sim}{\alpha, \varepsilon}}_{\alpha} \in C([0, T] ; \underset{\sim}{H}$ ) (cf. Lemma 1.2 on p. 176 of Temam [28]), whereas if $d=3$, then $u_{\alpha, \varepsilon}$ is weakly continuous only as a mapping from $[0, T]$ into $\underset{\sim}{H}$ (similarly as in Theorem 3.1 on p. 191 in Temam [28]). It is in the latter, weaker sense that the imposition of the initial condition to the $u_{\alpha, \varepsilon}$-equation will be understood for $d=2,3$ : that is, $\lim _{t \rightarrow 0}\left(\underset{\sim}{u_{\alpha, \varepsilon}}(\cdot, t), \underset{\sim}{v}(\cdot)\right)=\left({\underset{\sim}{u}}_{0}(\cdot), \underset{\sim}{v}(\cdot)\right)$ for all $\underset{\sim}{v} \in \underset{\sim}{H}$.

Throughout we will assume that $(2.21 \mathrm{a}, \mathrm{b})$ hold, with $\kappa \geq 5$ if $D$ is a bounded open ball in $\mathbb{R}^{d}$ so that (3.16a) holds. In addition, we assume that (2.22) and (3.7) hold. In order to prove existence of these weak solutions to $\left(\mathrm{P}_{\alpha, \varepsilon}\right)$, we consider a time semidiscretization. To this end, for any $T>0$, let $N \Delta t=T$ and $t_{n}=n \Delta t, n=0 \rightarrow$ $N$. An alternative approach would have been to use spatial semidiscretization, but the existence proof would have then required detailed knowledge of spectral properties of linear elliptic operators in Sobolev spaces with strongly degenerate weights. Time 
semidisretization is our preferred choice of technique, as it admits the use of an elliptic fixed-point argument at each time level.

In order to prove existence of weak solutions under minimal smoothness requirements on the initial data, we introduce ${\underset{\sim}{u}}^{0} \in \underset{\sim}{V}$ and $\psi^{0} \in L^{2}\left(\Omega \times D ; M^{-1}\right)$ as follows:

$$
{\underset{\sim}{u}}^{0}=S_{\Delta t} \underset{\sim}{u_{0}} \quad \text { and } \quad \psi^{0}=\frac{\psi_{0}}{1+\Delta t|q|^{2}} .
$$

Here we have adopted similar notation to that in $(3.10 \mathrm{a}, \mathrm{b})$. It follows immediately that $\psi^{0} \geq 0$ a.e. in $\Omega \times D$, and from (3.7) and (3.4a) that

$$
\int_{\Omega}\left[\left|u^{0}\right|^{2}+\Delta t\left|\underset{\sim}{\nabla}{\underset{\sim}{u}}^{0}\right|^{2}\right] \mathrm{d} \underset{\sim}{x}+\int_{\Omega \times D}\left(1+\Delta t|\underset{\sim}{\mid q}|^{2}\right) \frac{\left|\psi^{0}\right|^{2}}{M} \mathrm{~d} \underset{\sim}{q} \mathrm{~d} \underset{\sim}{x} \leq C .
$$

In addition, we have that ${\underset{\sim}{u}}^{0}$ converges to $\underset{\sim}{u_{0}}$ weakly in $\underset{\sim}{H}$ and $\psi^{0}$ converges to $\psi_{0}$ weakly in $L^{2}\left(\Omega \times D ; M^{-1}\right)$ as $\Delta t \rightarrow 0$.

As seen in [2], the noncorotational case is harder to analyze than the corotational case. Therefore in some places in the analysis below, we have to distinguish between these cases. For all $\underset{\sim}{v} \in W^{1, \infty}(\Omega)$, we introduce

$$
A(\underset{\sim}{v}):=\left\{\begin{array}{lll}
\left\|\nabla_{x} \chi\right\|_{L^{\infty}(\Omega)}^{2} & \text { if } & \underset{\sim}{g}(\cdot)=\bigotimes_{x} \cdot, \\
0 & \text { if } & \underset{\sim}{ }(\cdot)=\underset{\sim}{\Psi}(\cdot) .
\end{array}\right.
$$

It follows from (3.9b) that $A\left(J_{\alpha}^{x} \underset{\sim}{v}\right)$ is well defined for all $\underset{\sim}{v} \in L_{\sim}^{1}(\Omega)$.

Let $u_{\alpha, \varepsilon}^{0}=\underline{u}^{0}$ and $\psi_{\alpha, \varepsilon}^{0}=\psi^{0}$. Then, for $n=1 \rightarrow N$, given $\left\{u_{\alpha, \varepsilon}^{n-1}, A_{\alpha, \varepsilon}^{n-1}, \psi_{\alpha, \varepsilon}^{n-1}\right\} \in$ $\underset{\sim}{V} \times \mathbb{R}^{+} \times X_{q}^{+}$, where $A_{\alpha, \varepsilon}^{n-1}=A\left(\sim_{\alpha}^{x} \sim_{\alpha, \varepsilon}^{n-1}\right)$, find $\left\{\underset{\sim}{u_{\alpha, \varepsilon}^{n}}, A\left({\underset{\sim}{\alpha} \alpha}_{\alpha, \varepsilon}^{x} \sim_{\alpha, \varepsilon}^{n}\right), \psi_{\alpha, \varepsilon}^{n}, \underset{\sim}{C}\left(\psi_{\alpha, \varepsilon}^{n}\right)\right\} \in$ $\underset{\sim}{V} \times \mathbb{R}^{+} \times X_{q}^{+} \times \underset{\sim}{L^{2}}(\Omega)$ such that

$$
\begin{aligned}
& \int_{\Omega}\left[\frac{u_{\alpha, \varepsilon}^{n}-u_{\alpha, \varepsilon}^{n-1}}{\Delta t}+\left(\underset{\sim}{u_{\alpha, \varepsilon}^{n-1}} \cdot \underset{\sim}{\nabla_{x}}\right) \underset{\sim}{u_{\alpha, \varepsilon}^{n}}\right] \cdot \underset{\sim}{w} \mathrm{~d} x+\nu \int_{\Omega} \underset{\sim}{\nabla_{x}} \underset{\sim}{u_{\alpha, \varepsilon}^{n}}: \underset{\sim}{\nabla_{x}} \underset{\sim}{w} \mathrm{~d} x \\
& \left.=-k \mu \int_{\Omega} \underset{\approx}{\approx}\left(\psi_{\alpha, \varepsilon}^{n}\right): \underset{\approx}{\nabla_{x}} \underset{\sim}{I_{\alpha}} \underset{\sim}{w}\right) \mathrm{d} \underset{\sim}{\forall} \quad \underset{\sim}{w} \in \underset{\sim}{V},
\end{aligned}
$$

$$
\begin{aligned}
& \int_{\Omega \times D} \frac{\psi_{\alpha, \varepsilon}^{n}-\psi_{\alpha, \varepsilon}^{n-1}}{\Delta t} \frac{\varphi}{M} \mathrm{~d} q \mathrm{~d} x \\
& \left.+\int_{\Omega \times D}|q|_{\sim}^{2}\left[\left(1+\lambda A \underset{\sim}{A} \underset{\sim}{J_{\alpha}^{x}} u_{\alpha, \varepsilon}^{n}\right)\right) \psi_{\alpha, \varepsilon}^{n}-\left(1+\lambda A_{\alpha, \varepsilon}^{n-1}\right) \psi_{\alpha, \varepsilon}^{n-1}\right] \underset{\sim}{\frac{\varphi}{M}} \underset{\sim}{\mathrm{d} q} \mathrm{~d} x \\
& +\int_{\Omega \times D}\left[\frac{M}{2 \lambda} \underset{\sim}{\nabla_{q}}\left(\frac{\psi_{\alpha, \varepsilon}^{n}}{M}\right)-\left[\underset{\sim}{\sigma} \underset{\sim}{\left(J_{\alpha}^{x}\right.} \underset{\sim}{u} \sim_{\alpha, \varepsilon}^{n} \underset{\sim}{q}\right] \psi_{\alpha, \varepsilon}^{n}\right] \cdot \underset{\sim}{\nabla_{q}}\left(\frac{\varphi}{M}\right) \underset{\sim}{\mathrm{d} q} \mathrm{~d} x \\
& +\int_{\Omega \times D}\left[\varepsilon M \underset{\sim}{\underset{\sim}{\nabla}}\left(\frac{\psi_{\alpha, \varepsilon}^{n}}{M}\right)-\underset{\sim}{u_{\alpha, \varepsilon}^{n}} \psi_{\alpha, \varepsilon}^{n}\right] \cdot \underset{\sim}{\nabla_{x}}\left(\frac{\varphi}{M}\right) \underset{\sim}{\mathrm{d} q} \underset{\sim}{\mathrm{d} x}=0 \quad \forall \varphi \in X_{q} .
\end{aligned}
$$

$$
b\left(\sim_{\alpha, \varepsilon}^{n-1}\right)\left({\underset{\sim}{\alpha, \varepsilon}}_{\alpha, \varepsilon}^{n} \underset{\sim}{w}\right)=\int_{\Omega}\left[\underset{\sim \alpha, \varepsilon}{u-1} \cdot \underset{\sim}{w}-\Delta t k \mu \underset{\sim}{C}\left(\psi_{\alpha, \varepsilon}^{n}\right): \underset{\sim}{\nabla_{x}}(\underset{\sim}{I} \alpha \underset{\sim}{w})\right] \mathrm{d} \underset{\sim}{x} \quad \forall \underset{\sim}{w} \in \underset{\sim}{V},
$$


where for all $\underset{\sim}{v} \in \underset{\sim}{V}, \underset{\sim}{w_{i}} \in \underset{\sim}{H}{ }_{0}^{1}(\Omega), i=1,2$,

$$
\left.b\left(\underset{\sim}{v)} \underset{\sim}{w_{1}}, \underset{\sim}{w_{2}}\right):=\int_{\Omega}\left[\underset{\sim}{w_{1}}+\Delta t \underset{\sim}{v} \cdot \underset{\sim}{\nabla_{x}}\right) \underset{\sim}{w_{1}}\right] \cdot \underset{\sim}{w_{2}} \mathrm{~d} x+\Delta t \nu \int_{\Omega} \underset{\sim}{\nabla_{x}} \underset{\sim}{w_{1}}: \underset{\sim}{\nabla_{x}} \underset{\sim}{w_{2}} \mathrm{~d} x .
$$

It follows from (3.28) that

$$
\begin{aligned}
& \int_{\Omega}\left[\left(\underset{\sim}{v} \cdot \nabla_{x}\right) w_{1}\right] \cdot{\underset{\sim}{w}}_{2} \mathrm{~d} x \\
&=-\int_{\Omega}\left[\left(v_{\sim} \cdot \nabla_{x}\right) w_{2}\right] \cdot{\underset{\sim}{w}}_{1} \mathrm{~d} x \quad \forall \underset{\sim}{v} \in V, \quad \forall \underset{\sim}{w_{1}},{\underset{\sim}{w}}_{2} \in{\underset{\sim}{0}}_{0}^{1}(\Omega),
\end{aligned}
$$

and hence $b \underset{\sim}{v})(\cdot, \cdot)$ is a continuous and coercive bilinear functional on $\underset{\sim}{V} \times \underset{\sim}{V}$.

For $r>d$, let

$$
{\underset{\sim}{Y}}^{r}:=\left\{\underset{\sim}{v} \in L_{\sim}^{r}(\Omega): \int_{\Omega} \underset{\sim}{v} \cdot \nabla_{x} \underset{\sim}{w} \mathrm{~d} x \underset{\sim}{x}=0 \quad \forall \underset{\sim}{w} \in \underset{\sim}{W^{1, \frac{r}{r-1}}}(\Omega)\right\} .
$$

It is also convenient to rewrite $(3.26 \mathrm{~b})$ as

$$
a_{\alpha, \varepsilon}\left({\underset{\sim}{\alpha, \varepsilon}}_{\alpha, \varepsilon}^{n}\right)\left(\psi_{\alpha, \varepsilon}^{n}, \varphi\right)=\ell_{\alpha, \varepsilon}^{n}(\varphi) \quad \forall \varphi \in X_{q},
$$

where, for all $\varphi_{1}, \varphi_{2}, \varphi \in X_{q}$ and $\underset{\sim}{v} \in \underset{\sim}{Y^{r}}$,

$$
\begin{aligned}
& a_{\alpha, \varepsilon}(\underset{\sim}{v})\left(\varphi_{1}, \varphi_{2}\right):=\int_{\Omega \times D}\left(W\left({\underset{\sim}{J}}_{\alpha}^{x}\right) \varphi_{1} \varphi_{2}+\Delta t\left[\varepsilon M \nabla_{x}\left(\frac{\varphi_{1}}{M}\right)-\underset{\sim}{v} \varphi_{1}\right] \cdot \nabla_{x}\left(\frac{\varphi_{2}}{M}\right)\right. \\
& \ell_{\alpha, \varepsilon}^{n}(\varphi):=\int_{\Omega \times D} W_{\alpha, \varepsilon}^{n-1} \psi_{\alpha, \varepsilon}^{n-1} \varphi \mathrm{d} \underset{\sim}{q} \mathrm{~d} \underset{\sim}{ }
\end{aligned}
$$

and

$$
W(\underset{\sim}{v}):=\frac{1+\Delta t|\underset{\sim}{\mid q}|^{2}(1+\lambda A(\underset{\sim}{v}))}{M}, \quad W_{\alpha, \varepsilon}^{n-1}=\frac{1+\left.\Delta t \underset{\sim}{\mid q}\right|^{2}\left(1+\lambda A_{\alpha, \varepsilon}^{n-1}\right)}{M} .
$$

We have from Sobolev embedding that

$$
L^{s}\left(\Omega ; L^{2}(D ; M)\right) \subset H^{1}\left(\Omega ; L^{2}(D ; M)\right),
$$

where $s \in[1, \infty)$ if $d=2$ or $s \in[1,6]$ if $d=3$. It follows from (3.30) and (3.33) that for $r>d$

$$
\int_{\Omega \times D} \underset{\sim}{v} \varphi \cdot \nabla_{x}\left(\frac{\varphi}{M}\right) \mathrm{d} \underset{\sim}{\mathrm{d}} \mathrm{d} x=0 \quad \forall \underset{\sim}{v} \in \underset{\sim}{Y}{ }^{r}, \quad \forall \varphi \in X_{q} .
$$

In addition, it is easily deduced from $(3.25),(3.19 \mathrm{a})$, and $(3.12 \mathrm{~b})$ that $a_{\alpha, \varepsilon}(\underset{\sim}{v})(\cdot, \cdot)$ is a continuous nonsymmetric bilinear functional on $X_{q} \times X_{q}$ and $\ell_{\alpha}^{n}(\cdot)$ is a linear functional on $X_{q}$. Moreover, noting (3.34), and either noting (3.19a) in the corotational case 
or noting (3.25) and applying Young's inequality in the noncorotational case, we see that

$$
\begin{aligned}
& a_{\alpha, \varepsilon}(\underset{\sim}{v})(\varphi, \varphi) \\
\geq & \int_{\Omega \times D}\left[W_{c}|\varphi|^{2}+\Delta t \varepsilon M\left|\nabla_{x}\left(\frac{\varphi}{M}\right)\right|^{2}+\frac{\Delta t M}{4 \lambda}\left|\nabla_{q}\left(\frac{\varphi}{M}\right)\right|^{2}\right] \underset{\sim}{\mathrm{d}} \underset{\sim}{\mathrm{d}} \underset{\sim}{x} \quad \forall \varphi \in X_{q},
\end{aligned}
$$

where

$$
W_{c}:=\frac{1+\Delta t|\underset{\sim}{q}|^{2}}{M}
$$

Hence $a_{\alpha, \varepsilon}(\underset{\sim}{v})(\cdot, \cdot)$ is coercive on $X_{q} \times X_{q}$.

In order to prove existence of a solution to $(3.26 \mathrm{a}, \mathrm{b})$, we consider a fixed-point argument. Given $\underset{\sim}{\widehat{u}} \in \underset{\sim}{Y^{r}}$ with $r>d$, let $\left\{\psi^{\star},{\underset{\sim}{u}}^{\star}\right\} \in X_{q} \times \underset{\sim}{V}$ be such that

$$
\begin{aligned}
a_{\alpha, \varepsilon}(\underset{\sim}{\widehat{u}})\left(\psi^{\star}, \varphi\right) & =\ell_{\alpha, \varepsilon}^{n}(\varphi) \quad \forall \varphi \in X_{q} \\
b\left({\underset{\sim}{\alpha, \varepsilon}}_{\alpha, 1}^{n-1}\right)\left({\underset{\sim}{u}}^{\star}, \underset{\sim}{w}\right) & =\int_{\Omega}\left[{\underset{\sim}{\alpha, \varepsilon}}_{\alpha-1}^{n-1} \underset{\sim}{w}-\Delta t k \mu \underset{\approx}{C}\left(\psi^{\star}\right): \bigotimes_{x}(\underset{\sim}{I} \alpha \underset{\sim}{w})\right] \mathrm{d} \underset{\sim}{x} \quad \forall \underset{\sim}{w} \in \underset{\sim}{V},
\end{aligned}
$$

where, recalling $(3.9 \mathrm{~b}), J_{\alpha}^{x} \widehat{u} \in W^{1, \infty}(\Omega)$.

Noting (3.35), the Lax-Milgram theorem yields the existence of a unique solution to (3.37a). Noting (3.29), there exists a unique solution to (3.37b). Therefore the overall procedure $(3.37 \mathrm{a}, \mathrm{b})$ is well defined.

LEMMA 3.2. Let $\underset{\sim}{G}: \underset{\sim}{Y} \rightarrow \underset{\sim}{V} \subset \underset{\sim}{Y^{r}}, r \in(d, 6)$, denote the nonlinear map that takes $\underset{\sim}{\widehat{u}}$ to $\underset{\sim}{u^{\star}}=G \underset{\sim}{(\underset{\sim}{u})}$ via the procedure $(3.37 \mathrm{a}, \mathrm{b})$. Then $\underset{\sim}{G}$ has a fixed point. Hence there exists a solution $\left\{\underset{\sim \alpha, \varepsilon}{u_{\alpha, \varepsilon}^{n}}, A\left({\underset{\sim}{J}}_{\alpha}^{x} \underset{\alpha, \varepsilon}{u_{\alpha, \varepsilon}^{n}}\right), \psi_{\alpha, \varepsilon}^{n}, \underset{\approx}{C}\left(\psi_{\alpha, \varepsilon}^{n}\right)\right\} \in \underset{\sim}{V} \times \mathbb{R}^{+} \times X_{q}^{+} \times \underset{\approx}{L^{2}}(\Omega)$ to $(3.26 \mathrm{a}, \mathrm{b})$.

Proof. Clearly, a fixed point of $G$ yields a solution of $(3.26 \mathrm{a}, \mathrm{b})$. In order to show that $G$ has a fixed point, we apply Schauder's fixed-point theorem; that is, we need to show that (i) $\underset{\sim}{G}: \underset{\sim}{Y^{r}} \rightarrow \underset{\sim}{Y}{ }^{r}, r \in(d, 6)$, is continuous, that (ii) it is compact, and that (iii) there exists a $C_{\star} \in \mathbb{R}^{+}$such that

$$
\|\underset{\sim}{\widehat{u}}\|_{L^{r}(\Omega)} \leq C_{\star}
$$

for every $\underset{\sim}{\widehat{u}} \in \underset{\sim}{Y^{r}}$ and $\beta \in(0,1]$ satisfying $\underset{\sim}{\widehat{u}}=\beta G(\underset{\sim}{\widehat{u}})$.

Let $\left\{\widehat{\sim}^{(i)}\right\}_{i \geq 0}$ be such that

$$
{\underset{\sim}{\sim}}^{(i)} \in \underset{\sim}{Y} \rightarrow \underset{\sim}{\widehat{u}} \in \underset{\sim}{Y^{r}} \text { strongly in } \underset{\sim}{L^{r}}(\Omega) \quad \text { as } i \rightarrow \infty .
$$

We need to show that

$$
\widehat{\sim}^{(i)}:=G\left(\widehat{\sim}^{(i)}\right) \rightarrow \underset{\sim}{G}(\underset{\sim}{\widehat{u}}) \quad \text { strongly in }{\underset{\sim}{L}}^{r}(\Omega) \quad \text { as } i \rightarrow \infty,
$$

in order to prove (i) above. We have from the definition of $G$, see $(3.37 \mathrm{a}, \mathrm{b})$, that, for all $i \geq 0$,

(3.41a)

$$
b\left({\underset{\sim}{u, \varepsilon}}_{\alpha, 1}^{n-1}\right)\left(\widehat{\sim}^{(i)}, \underset{\sim}{w}\right)=\int_{\Omega}\left[{\underset{\sim}{u, \varepsilon}}_{\alpha, 1}^{n-1} \underset{\sim}{w}-\Delta t k \mu \underset{\sim}{C}\left(\widehat{\psi}^{(i)}\right): \underset{\sim}{\nabla}(\underset{\sim}{I} \underset{\sim}{w})\right] \mathrm{d} \underset{\sim}{x} \quad \forall \underset{\sim}{w} \in \underset{\sim}{V},
$$


where $\widehat{\psi}^{(i)} \in X_{q}$ satisfies

$$
a_{\alpha, \varepsilon}\left(\widehat{\sim}^{(i)}\right)\left(\widehat{\psi}^{(i)}, \varphi\right)=\ell_{\alpha, \varepsilon}^{n}(\varphi) \quad \forall \varphi \in X_{q},
$$

and from $(3.9 \mathrm{~b})$ we have that

$$
J_{\alpha}^{x} \widehat{\sim}^{(i)} \rightarrow J_{\alpha}^{x} \underset{\sim}{\widehat{u}} \text { strongly in } W^{1, \infty}(\Omega) \quad \text { as } i \rightarrow \infty .
$$

Choosing $\underset{\sim}{w} \equiv{\underset{\sim}{v}}^{(i)}$ in (3.41a), and noting (3.29), (3.9a), and (3.13), yields that, for all $i \geq 0, \widehat{\sim}^{(i)} \in \underset{\sim}{V}$ satisfies

$$
\begin{aligned}
& \int_{\Omega}\left[\left|\widehat{\sim}_{\sim}^{(i)}\right|^{2}+\left|\widehat{\sim}^{(i)}-{\underset{\sim}{\alpha, \varepsilon}}_{\alpha, \varepsilon}^{n-1}\right|^{2}-\left|\sim_{\alpha, \varepsilon}^{n-1}\right|^{2}\right] \mathrm{d} \underset{\sim}{x}+\Delta t \nu \int_{\Omega} \mid \underset{\sim}{\nabla_{x}}{\left.\underset{\sim}{\widehat{v}^{(i)}}\right|^{2} \mathrm{~d} \underset{\sim}{x}} \\
& \leq C \Delta t \int_{\Omega \times D} \frac{\left|\widehat{\psi}^{(i)}\right|^{2}}{M} \mathrm{~d} \underset{\sim}{q} \mathrm{~d} x,
\end{aligned}
$$

where we have noted the simple identity

$$
2\left(s_{1}-s_{2}\right) s_{1}=s_{1}^{2}+\left(s_{1}-s_{2}\right)^{2}-s_{2}^{2} \quad \forall s_{1}, s_{2} \in \mathbb{R} .
$$

Choosing $\varphi \equiv \widehat{\psi}^{(i)}$ in (3.41b), and noting (3.35) and (3.32b,c), yields, for all $i \geq 0$, that

$$
\begin{aligned}
\int_{\Omega \times D}\left[W_{c}\left|\widehat{\psi}^{(i)}\right|^{2}+2 \Delta t \varepsilon M\left|\nabla_{x}\left(\frac{\widehat{\psi}^{(i)}}{M}\right)\right|^{2}+\frac{\Delta t M}{2 \lambda}\left|\nabla_{q}\left(\frac{\widehat{\psi}^{(i)}}{M}\right)\right|^{2}\right] \underset{\sim}{q} \mathrm{~d} \underset{\sim}{ } \\
\leq\left(1+\lambda A_{\alpha, \varepsilon}^{n-1}\right)^{2} \int_{\Omega \times D} W_{c}\left|\psi_{\alpha, \varepsilon}^{n-1}\right|^{2} \mathrm{~d} \underset{\sim}{q} \mathrm{~d} x \leq C(\alpha) .
\end{aligned}
$$

Combining (3.42) and (3.44), and noting a well-known embedding result and a Poincaré inequality, we have for all $i \geq 0$ that

$$
\left\|\widehat{\sim}^{(i)}\right\|_{L^{r}(\Omega)} \leq C\left\|\nabla_{\sim} \widehat{\widehat{v}}^{(i)}\right\|_{L^{2}(\Omega)} \leq C(\alpha) .
$$

It follows from (3.44), (3.45), (3.13), and (2.4a), noting the compactness of the embedding $\underset{\sim}{H^{1}}(\Omega) \hookrightarrow \stackrel{\sim}{L}^{r}(\Omega), r \in(d, 6)$, that there exists a subsequence $\left\{\widehat{\psi}^{\left(i_{k}\right)}, \widehat{\sim}^{\left(i_{k}\right)}\right\}_{i_{k} \geq 0}$ and functions $\widehat{\psi} \in X_{q}$ and $\underset{\sim}{\widehat{v}} \in \underset{\sim}{V}$ such that as $i_{k} \rightarrow \infty$

$$
\begin{aligned}
& W_{c}^{\frac{1}{2}} \widehat{\psi}^{\left(i_{k}\right)} \rightarrow W_{c}^{\frac{1}{2}} \widehat{\psi} \quad \text { weakly in } L^{2}(\Omega \times D), \\
& M^{\frac{1}{2}} \underset{\sim}{\nabla_{x}}\left(\frac{\widehat{\psi}^{\left(i_{k}\right)}}{M}\right) \rightarrow M^{\frac{1}{2}} \underset{\sim}{\nabla_{x}}\left(\frac{\widehat{\psi}}{M}\right) \quad \text { weakly in } \underset{\sim}{L^{2}}(\Omega \times D), \\
& M^{\frac{1}{2}} \underset{\sim}{\nabla_{q}}\left(\frac{\widehat{\psi}^{\left(i_{k}\right)}}{M}\right) \rightarrow M^{\frac{1}{2}} \underset{\sim}{\nabla_{q}}\left(\frac{\widehat{\psi}}{M}\right) \quad \text { weakly in } \underset{\sim}{L^{2}}(\Omega \times D), \\
& \underset{\approx}{C}\left(\widehat{\psi}^{\left(i_{k}\right)}\right) \rightarrow \underset{\approx}{C}(\widehat{\psi}) \quad \text { weakly in } \underset{\approx}{L^{2}}(\Omega), \\
& \widehat{\sim}^{\left(i_{k}\right)} \rightarrow \underset{\sim}{\widehat{v}} \quad \text { weakly in }{\underset{\sim}{H}}^{1}(\Omega), \\
& \widehat{v}^{\left(i_{k}\right)} \rightarrow \underset{\sim}{\widehat{v}} \quad \text { strongly in } \underset{\sim}{L^{r}}(\Omega) .
\end{aligned}
$$


It follows from (3.41a), (3.28), and (3.46d,e) that $\underset{\sim}{\widehat{v}} \in \underset{\sim}{V}$ and $\widehat{\psi} \in X_{q}$ satisfy

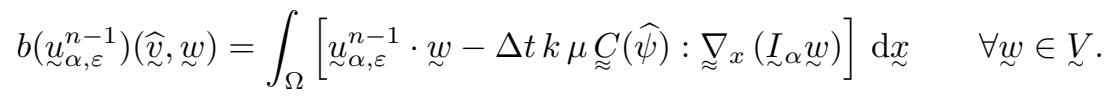

It follows from $(3.41 \mathrm{~b}, \mathrm{c}),(3.39),(3.32 \mathrm{a}-\mathrm{c}),(3.25),(3.46 \mathrm{a}-\mathrm{c})$, and (3.33) that $\underset{\sim}{\widehat{u}} \in \underset{\sim}{Y^{r}}$, $J_{\alpha}^{x} \widehat{\sim} \in W^{1, \infty}(\Omega)$, and $\widehat{\psi} \in X_{q}$ satisfy

$$
a_{\alpha, \varepsilon}(\widehat{\sim})(\widehat{\psi}, \varphi)=\ell_{\alpha, \varepsilon}^{n}(\varphi) \quad \forall \varphi \in X_{q} .
$$

Combining (3.48) and (3.47), we have that $\underset{\sim}{\widehat{v}}=G(\widehat{\sim}) \in \underset{\sim}{V}$. Therefore the whole sequence $\widehat{\widetilde{v}}^{(i)} \equiv G\left(\widehat{\widetilde{u}}^{(i)}\right) \rightarrow G(\widehat{u})$ strongly in $L^{r}(\Omega)$ as $i \rightarrow \infty$, and so (i) holds.

As the embedding $\underset{\sim}{V \hookrightarrow \underset{\sim}{L}} \sim^{r}(\Omega), r \in(d, 6)$, is compact, it follows that (ii) holds.

As regards (iii), $\underset{\sim}{\widehat{u}}=\beta \underset{\sim}{G(\underset{\sim}{\widehat{u}}) \text { implies that }\{\widehat{\psi}, \widehat{\sim}\}} \in X_{q} \times \underset{\sim}{V}$ satisfies

$$
\begin{array}{ll}
a_{\alpha, \varepsilon}(\widehat{\sim})(\widehat{\psi}, \varphi)=\ell_{\alpha, \varepsilon}^{n}(\varphi) & \forall \varphi \in X_{q}, \\
\left.b\left(\underset{\sim}{u_{\alpha, \varepsilon}^{n-1}}\right)(\underset{\sim}{\widehat{u}}, \underset{\sim}{w})=\beta \int_{\Omega}\left[{\underset{\sim}{\alpha, \varepsilon}}_{\alpha, 1}^{n-w}-\Delta t k \mu \underset{\sim}{C}(\widehat{\psi}): \underset{\sim}{\nabla_{x}} \underset{\sim}{\left(I_{\alpha} w\right.} \underset{\sim}{w}\right)\right] \underset{\sim}{\mathrm{d} x} \quad \underset{\sim}{\forall w} \in \underset{\sim}{V} .
\end{array}
$$

Choosing $\underset{\sim}{w} \equiv \underset{\sim}{\widehat{u}}$ in $(3.49 \mathrm{~b})$ yields, similarly to $(3.42)$, that

$$
\begin{aligned}
& \frac{1}{2} \int_{\Omega}\left[|\widehat{\sim}|^{2}+\left|\underset{\sim}{\widehat{u}}-\beta{\underset{\sim}{\alpha, \varepsilon}}_{\alpha-1}^{n-1}\right|^{2}-\beta^{2}\left|\sim_{\alpha, \varepsilon}^{n-1}\right|^{2}\right] \mathrm{d} x+\Delta t \nu \int_{\Omega}\left|\unrhd_{x} \underset{\sim}{\widehat{u}}\right|^{2} \mathrm{~d} x \\
& =-\Delta t \beta k \mu \int_{\Omega} \underset{\approx}{C}(\widehat{\psi}): \underset{\approx}{\nabla_{x}}(\underset{\sim}{I} \alpha \underset{\sim}{\widehat{u}}) \mathrm{d} x \leq C \Delta t \int_{\Omega \times D} \frac{|\widehat{\psi}|^{2}}{M} \mathrm{~d} \underset{\sim}{\mathrm{d}} \mathrm{d} x .
\end{aligned}
$$

Choosing $\varphi=\widehat{\psi}$ in (3.49a) yields, similarly to (3.44), that

$$
\begin{aligned}
\int_{\Omega \times D}\left[W_{c}|\widehat{\psi}|^{2}+2 \Delta t \varepsilon M \mid \nabla_{x}\right. & \left.\left.\left(\frac{\widehat{\psi}}{M}\right)\right|^{2}+\frac{\Delta t M}{2 \lambda}\left|\nabla_{q}\left(\frac{\widehat{\psi}}{M}\right)\right|^{2}\right] \underset{\sim}{\mathrm{d} q} \mathrm{~d} \underset{\sim}{x} \\
& \leq\left(1+\lambda A_{\alpha, \varepsilon}^{n-1}\right)^{2} \int_{\Omega \times D} W_{c}\left|\psi_{\alpha, \varepsilon}^{n-1}\right|^{2} \mathrm{~d} \underset{\sim}{q} \mathrm{~d} \underset{\sim}{x} \leq C(\alpha) .
\end{aligned}
$$

Combining (3.50) and (3.51), and noting the embedding $\underset{\sim}{V} \underline{\sim}^{r}(\Omega)$, gives rise to the desired bound (3.38) with $C$ dependent on $\Delta t$ and $\alpha$. Hence (iii) holds, and so $G$ has a fixed point. Finally, as $\psi_{\alpha, \varepsilon}^{n-1} \in X_{q}^{+}$and $\psi_{\alpha, \varepsilon}^{n} \in X_{q} \Longrightarrow\left[\psi_{\alpha, \varepsilon}^{n}\right]_{-} \in X_{q}$, recall (3.17), it follows from $(3.31)$ and $(3.32 \mathrm{a}, \mathrm{b})$ that

$$
a_{\alpha, \varepsilon}\left(\underset{\sim}{u_{\alpha, \varepsilon}^{n}}\right)\left(\left[\psi_{\alpha, \varepsilon}^{n}\right]_{-},\left[\psi_{\alpha, \varepsilon}^{n}\right]_{-}\right)=a_{\alpha, \varepsilon}\left(\underset{\sim}{u_{\alpha, \varepsilon}^{n}}\right)\left(\psi_{\alpha, \varepsilon}^{n},\left[\psi_{\alpha, \varepsilon}^{n}\right]_{-}\right)=\ell_{\alpha, \varepsilon}^{n}\left(\left[\psi_{\alpha, \varepsilon}\right]_{-}\right) \leq 0 .
$$

Therefore (3.35) yields that $\left[\psi_{\alpha, \varepsilon}^{n}\right]_{-}=0$; that is, $\psi_{\alpha, \varepsilon}^{n} \in X_{q}^{+}$. Thus we have proved existence of a solution to $(3.26 \mathrm{a}, \mathrm{b})$.

Choosing $\underset{\sim}{w} \equiv u_{\alpha, \varepsilon}^{n}$ in (3.27) yields, similarly to (3.50), that

$$
\begin{aligned}
& \frac{1}{2} \int_{\Omega}\left[\left|u_{\alpha, \varepsilon}^{n}\right|^{2}+\left|u_{\alpha, \varepsilon}^{n}-{\underset{\sim}{u, \varepsilon}}_{\alpha-1}^{n-1}\right|^{2}-\left|u_{\sim \alpha, \varepsilon}^{n-1}\right|^{2}\right] \mathrm{d} x \underset{\sim}{x}+\Delta t \nu \int_{\Omega}\left|\nabla_{x} \underset{\sim \alpha, \varepsilon}{u_{\alpha}^{n}}\right|^{2} \mathrm{~d} x
\end{aligned}
$$

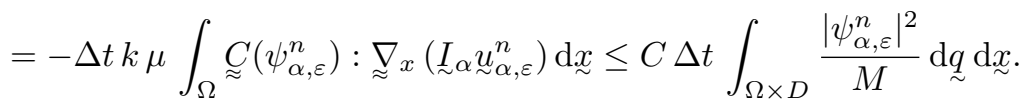


Choosing $\underset{\sim}{w} \equiv S_{\gamma}\left(\frac{u_{\alpha, \varepsilon}^{n}-u_{\alpha, \varepsilon}^{n-1}}{\Delta t}\right) \in \underset{\sim}{V}$ in (3.27) yields, noting (3.3), (3.20), (3.9a), and (3.29), that

$$
\begin{aligned}
& \int_{\Omega}\left[\gamma\left|\nabla_{\approx}\left[\underset{\sim}{S_{\gamma}}\left(\frac{\underset{u_{\alpha, \varepsilon}^{n}-u_{\alpha, \varepsilon}^{n-1}}{\sim}}{\Delta t}\right)\right]\right|^{2}+\left|\underset{\sim}{S_{\gamma}}\left(\frac{\underset{\sim}{u_{\alpha, \varepsilon}^{n}-u_{\alpha, \varepsilon}^{n-1}}}{\Delta t}\right)\right|^{2}\right] \underset{\sim}{\mathrm{d} x} \\
& =-\nu \int_{\Omega} \underset{\approx}{\nabla_{x}} u_{\sim \alpha, \varepsilon}^{n}: \underset{\approx}{\nabla_{x}}\left[\underset{\sim}{S_{\gamma}}\left(\frac{\underset{\sim}{u_{\alpha, \varepsilon}^{n}-u_{\alpha, \varepsilon}^{n-1}}}{\Delta t}\right)\right] \mathrm{d} x \\
& +\int_{\Omega} \underset{\approx}{C}\left(\psi_{\alpha, \varepsilon}^{n}\right): \underset{\approx}{\nabla_{x}}\left(\underset{\sim}{I_{\alpha}}\left[\underset{\sim}{S_{\gamma}}\left(\frac{u_{\alpha, \varepsilon}^{n}-u_{\alpha, \varepsilon}^{n-1}}{\Delta t}\right)\right]\right) \underset{\sim}{\sim}
\end{aligned}
$$

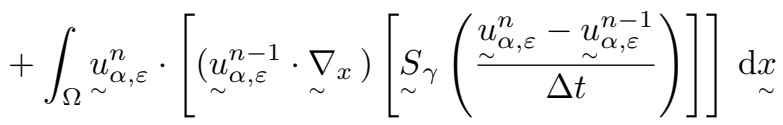

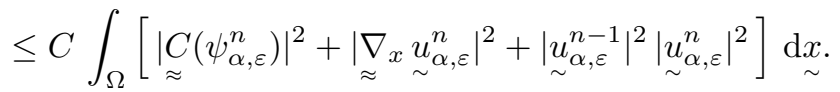

Applying the Cauchy-Schwarz inequality, the algebraic-geometric mean inequality, and a Gagliardo-Nirenberg inequality yields that

$$
\begin{aligned}
\int_{\Omega}\left|\sim_{\alpha, \varepsilon}^{n-1}\right|^{2}\left|u_{\alpha, \varepsilon}^{n}\right|^{2} \mathrm{~d} x & \leq\left(\int_{\Omega}\left|\sim_{\alpha, \varepsilon}^{n-1}\right|^{4} \mathrm{~d} x\right)^{\frac{1}{2}}\left(\int_{\Omega}\left|u_{\alpha, \varepsilon}^{n}\right|^{4} \mathrm{~d} \underset{\sim}{x}\right)^{\frac{1}{2}} \leq \frac{1}{2} \sum_{m=n-1}^{n} \int_{\Omega}\left|u_{\alpha, \varepsilon}^{m}\right|^{4} \mathrm{~d} x \\
& \leq C \sum_{m=n-1}^{n}\left[\left(\int_{\Omega}\left|u_{\sim, \varepsilon}^{m}\right|^{2} \mathrm{~d} x\right)^{2-\frac{d}{2}}\left(\int_{\Omega}\left|\nabla_{x}{\underset{\sim}{u} \alpha, \varepsilon}^{m}\right|^{2} \mathrm{~d} x\right)^{\frac{d}{2}}\right] .
\end{aligned}
$$

First we consider the corotational case for (3.31), where we can exploit (3.19a). Choosing $\varphi=\psi_{\alpha, \varepsilon}^{n}$ in (3.31), and noting (3.43), (3.25), (3.34), and (3.19a), yields that

$$
\begin{aligned}
& \int_{\Omega \times D} W_{c}\left[\left|\psi_{\alpha, \varepsilon}^{n}\right|^{2}+\left|\psi_{\alpha, \varepsilon}^{n}-\psi_{\alpha, \varepsilon}^{n-1}\right|^{2}\right] \mathrm{d} \underset{\sim}{\mathrm{d}} \mathrm{\sim} \underset{\sim}{x}+2 \Delta t \varepsilon \int_{\Omega \times D} M\left|\nabla_{x}\left(\frac{\psi_{\alpha, \varepsilon}^{n}}{M}\right)\right|^{2} \mathrm{~d} \underset{\sim}{q} \mathrm{~d} \underset{\sim}{x} \\
& +\frac{\Delta t}{\lambda} \int_{\Omega \times D} M\left|\nabla_{q}\left(\frac{\psi_{\alpha, \varepsilon}^{n}}{M}\right)\right|^{2} \mathrm{~d} \underset{\sim}{\mathrm{d}} \mathrm{d} x=\int_{\Omega \times D} W_{c}\left|\psi_{\alpha, \varepsilon}^{n-1}\right|^{2} \mathrm{~d} \underset{\sim}{q} \mathrm{~d} \underset{\sim}{.} .
\end{aligned}
$$

Summing (3.53) and (3.56) from $n=1 \rightarrow m$, with $1 \leq m \leq N$, and noting (3.24) 
yields that

$$
\begin{aligned}
& \max _{n=0 \rightarrow N}\left[\int_{\Omega \times D} W_{c}\left|\psi_{\alpha, \varepsilon}^{n}\right|^{2} \mathrm{~d} \underset{\sim}{\mathrm{d}} \mathrm{d} \underset{\sim}{x}\right]+\frac{1}{\lambda} \sum_{n=1}^{N} \Delta t \int_{\Omega \times D} M\left|\nabla_{q}\left(\frac{\psi_{\alpha, \varepsilon}^{n}}{M}\right)\right|^{2} \mathrm{~d} \underset{\sim}{q} \mathrm{~d} \underset{\sim}{x} \\
& +\varepsilon \sum_{n=1}^{N} \Delta t \int_{\Omega \times D} M\left|\nabla_{x}\left(\frac{\psi_{\alpha, \varepsilon}^{n}}{M}\right)\right|^{2} \mathrm{~d} \underset{\sim}{q} \mathrm{~d} \underset{\sim}{x}+\sum_{n=1}^{N} \int_{\Omega \times D} W_{c}\left|\psi_{\alpha, \varepsilon}^{n}-\psi_{\alpha, \varepsilon}^{n-1}\right|^{2} \mathrm{~d} \underset{\sim}{q} \mathrm{~d} \underset{\sim}{\sim} \\
& +\max _{n=0 \rightarrow N}\left[\int_{\Omega}\left|\underset{\widetilde{\sim}}{C}\left(\psi_{\alpha, \varepsilon}^{n}\right)\right|^{2} \mathrm{~d} x\right] \\
& \leq C \int_{\Omega \times D} W_{c}\left|\psi^{0}\right|^{2} \mathrm{~d} \underset{\sim}{q} \mathrm{~d} x \leq C, \\
& \max _{n=0 \rightarrow N}\left[\int_{\Omega}\left|u_{\sim \alpha, \varepsilon}^{n}\right|^{2} \mathrm{~d} x\right]+\sum_{n=1}^{N} \int_{\Omega}\left|u_{\alpha, \varepsilon}^{n}-u_{\alpha, \varepsilon}^{n-1}\right|^{2} \mathrm{~d} x+\nu \sum_{n=1}^{N} \Delta t \int_{\Omega}\left|\nabla_{x} \underset{\sim \alpha, \varepsilon}{n}\right|^{2} \mathrm{~d} x \\
& \leq C \int_{\Omega}\left|{\underset{u}{u}}^{0}\right|^{2} \mathrm{~d} \underset{\sim}{x}+C T \int_{\Omega \times D} W_{c}\left|\psi^{0}\right|^{2} \mathrm{~d} \underset{\sim}{q} \mathrm{~d} \underset{\sim}{x} \leq C(T) .
\end{aligned}
$$

In addition, taking the $\frac{2}{d}$ power of both sides of (3.54), summing from $n=1 \rightarrow N$, and noting (3.55), (3.57a,b), and (3.24) yields that

$$
\begin{aligned}
& \sum_{n=1}^{N} \Delta t\left(\int_{\Omega}\left[\gamma\left|\underset{\sim}{\nabla_{x}}\left[\underset{\sim}{S_{\gamma}}\left(\frac{u_{\alpha, \varepsilon}^{n}-u_{\alpha, \varepsilon}^{n-1}}{\Delta t}\right)\right]\right|^{2}+\left|\underset{\sim}{S_{\gamma}}\left(\frac{\underset{\sim}{u_{\alpha, \varepsilon}^{n}-u_{\alpha, \varepsilon}^{n-1}}}{\Delta t}\right)\right|^{2}\right] \underset{\sim}{\mathrm{d} x}\right)^{\frac{2}{d}}
\end{aligned}
$$

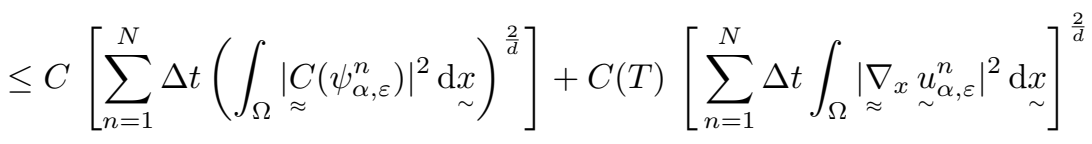

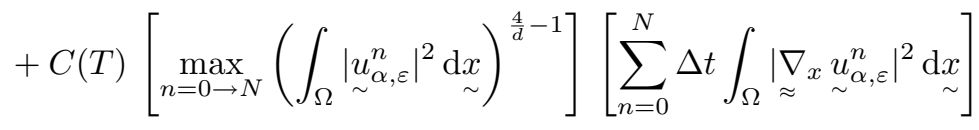$$
\leq C(T) \text {. }
$$

We now consider the noncorotational case for (3.31), where at first we have to apply a different testing procedure to $\varphi=\psi_{\alpha, \varepsilon}^{n}$ than the one used in the corotational case. Choosing $\varphi=M$ in (3.31) yields that

$$
\begin{aligned}
\left.\int_{\Omega \times D}\left[1+\Delta t \mid \underset{\sim}{|q|^{2}}\left(1+\lambda \underset{\sim}{A} \underset{\sim}{J_{\alpha}^{x}} u_{\alpha, \varepsilon}^{n}\right)\right)\right] \psi_{\alpha, \varepsilon}^{n} \underset{\sim}{\mathrm{d} q} \mathrm{~d} x \\
=\int_{\Omega \times D}\left[1+\Delta t \underset{\sim}{|q|^{2}}\left(1+\lambda A_{\alpha, \varepsilon}^{n-1}\right)\right] \underset{\alpha, \varepsilon}{\psi_{\sim}^{n-1}} \underset{\sim}{\mathrm{d} q} \mathrm{~d} x .
\end{aligned}
$$


Choosing $\varphi=U M$ in (3.31), and noting (2.10), (2.4a), (3.16a,b), (2.9), and (2.20), yields that

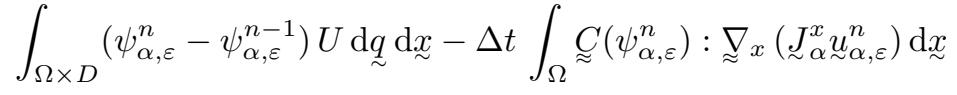

$$
\begin{aligned}
& +\Delta t \int_{\Omega \times D}|q|^{2}\left[\left(1+\lambda A\left(J_{\alpha \sim \alpha, \varepsilon}^{x} u_{\alpha, \varepsilon}^{n}\right)\right) \psi_{\alpha, \varepsilon}^{n}-\left(1+\lambda A_{\alpha, \varepsilon}^{n-1}\right) \psi_{\alpha, \varepsilon}^{n-1}\right] U \mathrm{~d} \underset{\sim}{\mathrm{d}} \underset{\sim}{x} \\
& =-\frac{\Delta t}{2 \lambda} \int_{\Omega \times D} M \underset{\sim}{\nabla_{q}}\left(\frac{\psi_{\alpha, \varepsilon}^{n}}{M}\right) \cdot U^{\prime} \underset{\sim}{q} \mathrm{~d} \underset{\sim}{q} \mathrm{~d} x \\
& =\frac{\Delta t}{2 \lambda} \int_{\Omega \times D}\left[\left(U^{\prime \prime}-\left(U^{\prime}\right)^{2}\right)|\underset{\sim}{q}|^{2}+d U^{\prime}\right] \psi_{\alpha, \varepsilon}^{n} \mathrm{~d} \underset{\sim}{q} \mathrm{~d} \underset{\sim}{x} \\
& \leq \frac{-\Delta t c_{2}}{2 \lambda} \int_{\Omega \times\left\{|\underset{\sim}{q}|^{2} \geq \frac{d}{c_{2}}\right\}}|\underset{\sim}{q}|^{2} U^{\prime} \psi_{\alpha, \varepsilon}^{n} \mathrm{~d} \underset{\sim}{q} \mathrm{~d} \underset{\sim}{x}+\frac{\Delta t d}{2 \lambda} \int_{\Omega \times\left\{|\underset{\sim}{\mid q}|^{2} \leq \frac{d}{c_{2}}\right\}} U^{\prime} \psi_{\alpha, \varepsilon}^{n} \mathrm{~d} \underset{\sim}{\mathrm{d}} \mathrm{d} x \\
& \leq \frac{-\Delta t c_{2}}{2 \lambda} \int_{\Omega \times\left\{|q|^{2} \geq \frac{d}{c_{2}}\right\}}|\underset{\sim}{\mid q}|^{2} U^{\prime} \psi_{\alpha, \varepsilon}^{n} \mathrm{~d} \underset{\sim}{q} \mathrm{~d} \underset{\sim}{x} \\
& +C \Delta t \int_{\Omega \times D}\left[1+\Delta t|\underset{\sim}{q}|^{2}\left(1+\lambda A\left(J_{\alpha}^{x} \sim_{\alpha, \varepsilon}^{n}\right)\right)\right] \psi_{\alpha, \varepsilon}^{n} \mathrm{~d} \underset{\sim}{q} \mathrm{~d} \underset{\sim}{ } .
\end{aligned}
$$

Combining (3.53) in the noncorotational case and (3.60) multiplied by $k \mu$, and noting (3.59), yields that

$$
\begin{aligned}
& \frac{1}{2} \int_{\Omega}\left[\left|u_{\alpha, \varepsilon}^{n}\right|^{2}+\left|u_{\alpha, \varepsilon}^{n}-{\underset{\sim}{u, \varepsilon}}_{\alpha, 1}^{n-1}\right|^{2}\right] \mathrm{d} x_{\sim}+\Delta t \nu \int_{\Omega}\left|{\underset{\sim}{\nabla}}_{x}{\underset{\sim}{u, \varepsilon}}_{\alpha, \varepsilon}^{n}\right|^{2} \mathrm{~d} x \\
& +k \mu \int_{\Omega \times D}\left[1+\Delta t|q|^{2}\left(1+\lambda A\left(J_{\alpha}^{x} u_{\alpha, \varepsilon}^{n}\right)\right)\right] U \psi_{\alpha, \varepsilon}^{n} \mathrm{~d} \underset{\sim}{q} \mathrm{~d} \underset{\sim}{x}
\end{aligned}
$$

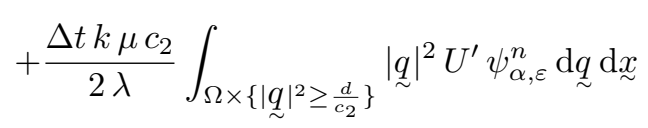

$$
\begin{aligned}
& \leq \frac{1}{2} \int_{\Omega}\left|u_{\alpha, \varepsilon}^{n-1}\right|^{2} \mathrm{~d} \underset{\sim}{x}+k \mu \int_{\Omega \times D}\left[1+\Delta t|q|^{2}\left(1+\lambda A_{\alpha, \varepsilon}^{n-1}\right)\right] U \psi_{\alpha, \varepsilon}^{n-1} \mathrm{~d} \underset{\sim}{\mathrm{d}} \mathrm{d} \underset{\sim}{x} \\
& +C k \mu \Delta t \int_{\Omega \times D}\left[1+\Delta t|q|^{2}\left(1+\lambda A_{\alpha, \varepsilon}^{n-1}\right)\right] \psi_{\alpha, \varepsilon}^{n-1} \mathrm{~d} \underset{\sim}{q} \mathrm{~d} \underset{\sim}{x} .
\end{aligned}
$$

Summing this from $n=1 \rightarrow m, m=1 \rightarrow N$, and noting by induction on (3.59) that

$$
\begin{aligned}
& \int_{\Omega \times D}\left[1+\Delta t|\underset{\sim}{q}|^{2}\left(1+\lambda A_{\alpha, \varepsilon}^{n-1}\right)\right] \psi_{\alpha, \varepsilon}^{n-1} \mathrm{~d} \underset{\sim}{q} \mathrm{~d} \underset{\sim}{x} \\
&=\int_{\Omega \times D}\left[1+\Delta t|\underset{\sim}{\mid q}|^{2}\left(1+\lambda A_{\alpha, \varepsilon}^{0}\right)\right] \psi^{0} \mathrm{~d} \underset{\sim}{q} \mathrm{~d} \underset{\sim}{x}
\end{aligned}
$$


and (3.24), yields that

$$
\begin{aligned}
& \max _{n=0 \rightarrow N}\left[\left.\int_{\Omega}\left|u_{\sim}^{n}\right|^{2}\right|^{2} \mathrm{~d} x\right]+\sum_{n=1}^{N} \int_{\Omega}\left|\underset{\sim}{u_{\alpha, \varepsilon}^{n}}-\underset{\sim}{u_{\alpha, \varepsilon}^{n-1}}\right|^{2} \mathrm{~d} \underset{\sim}{x} \\
& +\nu \sum_{n=1}^{N} \Delta t \int_{\Omega}\left|\underset{\sim}{\nabla_{x}} \underset{\sim}{u_{\alpha, \varepsilon}^{n}}\right|^{2} \mathrm{~d} x+k \mu \max _{n=1 \rightarrow N}\left[\int_{\Omega \times D} U \psi_{\alpha, \varepsilon}^{n} \underset{\sim}{\mathrm{d} q} \underset{\sim}{\mathrm{d} x}\right] \\
& +\frac{k \mu c_{2}}{2 \lambda} \sum_{n=1}^{N} \Delta t \int_{\Omega \times\left\{|q|^{2} \geq \frac{d}{c_{2}}\right\}} \underset{\sim}{|q|^{2}} U^{\prime} \psi_{\alpha, \varepsilon}^{n} \underset{\sim}{\sim} \mathrm{d} \underset{\sim}{\mathrm{d} x} \\
& \left.+k \mu \Delta t \max _{n=1 \rightarrow N}\left[\int_{\Omega \times D}|q|^{2}\left(1+\lambda A \underset{\sim}{A} \underset{\sim}{J_{\alpha}^{x}} u_{\alpha, \varepsilon}^{n}\right)\right) U \psi_{\alpha, \varepsilon}^{n} \underset{\sim}{\mathrm{d} q} \mathrm{~d} x\right] \\
& \leq C \int_{\Omega}\left|u_{\sim}^{0}\right|^{2} \mathrm{~d} x+C(T) \int_{\Omega \times D}\left[1+\Delta t \underset{\sim}{|q|^{2}}\left(1+\lambda A_{\alpha, \varepsilon}^{0}\right)\right](1+U) \underset{\sim}{\psi^{0} \mathrm{~d} q} \underset{\sim}{\mathrm{d} x} \\
& \leq C(T) \text {. }
\end{aligned}
$$

The bounds on $\psi_{\alpha, \varepsilon}^{n}$ in (3.63) for the noncorotational case do not suffice in order to pass to the limit $\Delta t \rightarrow 0$ in the summation over $n$ of (3.26b). One needs to establish additional bounds on $\psi_{\alpha, \varepsilon}^{n}$. We confine ourselves to the physically more realistic case of FENE-type models, i.e., $D$ bounded, for the general noncorotational case.

It follows from $(3.25),(3.9 \mathrm{~b})$, and (3.63) that

$$
\begin{aligned}
\sum_{n=1}^{N} \Delta t A_{\alpha, \varepsilon}^{n-1} & =\sum_{n=1}^{N} \Delta t\left\|\nabla_{x}\left(J_{\alpha}^{x} u_{\alpha, \varepsilon}^{n-1}\right)\right\|_{L^{\infty}(\Omega)}^{2} \leq C(\alpha) \sum_{n=1}^{N} \Delta t\left\|u_{\alpha, \varepsilon}^{n-1}\right\|_{L^{2}(\Omega)}^{2} \\
& \leq C_{1}(\alpha, T) .
\end{aligned}
$$

Choosing $\varphi=\psi_{\alpha, \varepsilon}^{n}$ in (3.31) and noting (3.32c), (3.36), and (3.35) yields that

$$
\begin{aligned}
& \int_{\Omega \times D} W_{c} \psi_{\alpha, \varepsilon}^{n}\left(\psi_{\alpha, \varepsilon}^{n}-\psi_{\alpha, \varepsilon}^{n-1}\right) \mathrm{d} \underset{\sim}{q} \mathrm{~d} \underset{\sim}{x} \\
& +\Delta t \varepsilon \int_{\Omega \times D} M\left|\nabla_{x}\left(\frac{\psi_{\alpha, \varepsilon}^{n}}{M}\right)\right|^{2} \underset{\sim}{\mathrm{d} q} \mathrm{~d} \underset{\sim}{x}+\frac{\Delta t}{4 \lambda} \int_{\Omega \times D} M\left|\nabla_{q}\left(\frac{\psi_{\alpha, \varepsilon}^{n}}{M}\right)\right|^{2} \mathrm{~d} \underset{\sim}{\mathrm{d}} \mathrm{d} \underset{\sim}{\sim} \\
& \leq \Delta t \lambda A_{\alpha, \varepsilon}^{n-1} \int_{\Omega \times D} \frac{|\underset{\sim}{\mid q}|^{2}}{M} \psi_{\alpha, \varepsilon}^{n} \psi_{\alpha, \varepsilon}^{n-1} \mathrm{~d} \underset{\sim}{q} \mathrm{~d} \underset{\sim}{x} .
\end{aligned}
$$

Applying the identity (3.43) and Young's inequality to (3.65), and noting that $D$ is bounded and (3.64), with $C_{1} \equiv C_{1}(\alpha, T)$, yields that

$$
\begin{aligned}
& \left(1-\frac{1}{2} C_{1}^{-1} \Delta t A_{\alpha, \varepsilon}^{n-1}\right) \int_{\Omega \times D} W_{c}\left|\psi_{\alpha, \varepsilon}^{n}\right|^{2} \mathrm{~d} \underset{\sim}{q} \mathrm{~d} \underset{\sim}{x}+\int_{\Omega \times D} W_{c}\left|\psi_{\alpha, \varepsilon}^{n}-\psi_{\alpha, \varepsilon}^{n-1}\right|^{2} \mathrm{~d} \underset{\sim}{q} \mathrm{~d} \underset{\sim}{x} \\
& +2 \Delta t \varepsilon \int_{\Omega \times D} M\left|\nabla_{x}\left(\frac{\psi_{\alpha, \varepsilon}^{n}}{M}\right)\right|^{2} \mathrm{~d} \underset{\sim}{q} \mathrm{~d} \underset{\sim}{x}+\frac{\Delta t}{2 \lambda} \int_{\Omega \times D} M\left|\nabla_{q}\left(\frac{\psi_{\alpha, \varepsilon}^{n}}{M}\right)\right|^{2} \mathrm{~d} \underset{\sim}{q} \mathrm{~d} \underset{\sim}{x} \\
& (3.66) \leq\left(1+C_{2} \Delta t A_{\alpha, \varepsilon}^{n-1}\right) \int_{\Omega \times D} W_{c}\left|\psi_{\alpha, \varepsilon}^{n-1}\right|^{2} \mathrm{~d} \underset{\sim}{q} \mathrm{~d} \underset{\sim}{x},
\end{aligned}
$$


where $C_{2}(\alpha, T)$. It follows from (3.66) and (3.64) that

$$
\begin{aligned}
\int_{\Omega \times D} W_{c}\left|\psi_{\alpha, \varepsilon}^{n}\right|^{2} \mathrm{~d} q \underset{\sim}{\mathrm{d}} \underset{\sim}{x} & \leq \frac{1+C_{2} \Delta t A_{\alpha, \varepsilon}^{n-1}}{1-\frac{1}{2} C_{1}^{-1} \Delta t A_{\alpha, \varepsilon}^{n-1}} \int_{\Omega \times D} W_{c}\left|\psi_{\alpha, \varepsilon}^{n-1}\right|^{2} \mathrm{~d} \underset{\sim}{q} \mathrm{~d} \underset{\sim}{x} \\
& \leq \mathrm{e}^{C(\alpha, T) \Delta t A_{\alpha, \varepsilon}^{n-1}} \int_{\Omega \times D} W_{c}\left|\psi_{\alpha, \varepsilon}^{n-1}\right|^{2} \mathrm{~d} \underset{\sim}{q} \mathrm{~d} \underset{\sim}{x} .
\end{aligned}
$$

Hence combining (3.67) and (3.64), summing (3.66) from $n=1 \rightarrow N$, and noting (3.13) and (3.24) yields the bounds (3.57a) for the general noncorotational FENE model; in particular,

$$
\begin{gathered}
\max _{n=0 \rightarrow N}\left[\int_{\Omega \times D} W_{c}\left|\psi_{\alpha, \varepsilon}^{n}\right|^{2} \mathrm{~d} \underset{\sim}{q} \mathrm{~d} \underset{\sim}{x}\right]+\varepsilon \sum_{n=1}^{N} \Delta t \int_{\Omega \times D} M\left|\nabla_{x}\left(\frac{\psi_{\alpha, \varepsilon}^{n}}{M}\right)\right|^{2} \mathrm{~d} \underset{\sim}{q} \mathrm{~d} \underset{\sim}{x} \\
+\frac{1}{\lambda} \sum_{n=1}^{N} \Delta t \int_{\Omega \times D} M\left|\nabla_{q}\left(\frac{\psi_{\alpha, \varepsilon}^{n}}{M}\right)\right|^{2} \mathrm{~d} \underset{\sim}{q} \mathrm{~d} \underset{\sim}{x}+\sum_{n=1}^{N} \int_{\Omega \times D} W_{c}\left|\psi_{\alpha, \varepsilon}^{n}-\psi_{\alpha, \varepsilon}^{n-1}\right|^{2} \mathrm{~d} \underset{\sim}{q} \mathrm{~d} \underset{\sim}{\sim} \\
\quad+\max _{n=0 \rightarrow N}\left[\int_{\Omega}\left|\underset{\sim}{C}\left(\psi_{\alpha, \varepsilon}^{n}\right)\right|^{2} \mathrm{~d} \underset{\sim}{x}\right] \leq C(\alpha, T) .
\end{gathered}
$$

Finally, taking the $\frac{2}{d}$ power of both sides of (3.54), summing from $n=1 \rightarrow N$, and noting (3.55), (3.63), (3.68), and (3.24) yields, similarly to (3.58), that

$$
\sum_{n=1}^{N} \Delta t\left(\int_{\Omega}\left[\gamma\left|\nabla_{x}\left[{\underset{\sim}{\gamma}}_{\gamma}\left(\frac{u_{\alpha, \varepsilon}^{n}-u_{\alpha, \varepsilon}^{n-1}}{\Delta t}\right)\right]\right|^{2}+\left|{\underset{\sim}{\gamma}}_{\gamma}\left(\frac{u_{\alpha, \varepsilon}^{n}-u_{\alpha, \varepsilon}^{n-1}}{\Delta t}\right)\right|^{2}\right] \mathrm{d} x\right)^{\frac{2}{d}}
$$

$$
\leq C(\alpha, T) \text {. }
$$

We have now established all of the analogues of the bounds (3.57a,b) and (3.58) in the corotational case for the general noncorotational FENE-type potentials; see (3.63), (3.68), and (3.69) above. The key difference is that the corotational bounds are independent of $\alpha>0$, whereas the noncorotational bounds (3.68) and (3.69) are $\alpha$ dependent.

Finally, we note that in the corotational case one can derive an upper bound, in addition to the zero lower bound, on $\psi_{\alpha, \varepsilon}^{n}$. To do so, we proceed inductively. Assuming that for some $L^{n-1} \in \mathbb{R}^{+}, \psi_{\alpha, \varepsilon}^{n-1} \leq L^{n-1} M$ a.e. in $\Omega \times D$, we then determine $L^{n} \in \mathbb{R}^{+}$ in terms of $L^{n-1}$ such that $\psi_{\alpha, \varepsilon}^{n} \leq L^{n} M$ a.e. in $\Omega \times D$. Now, from (3.17), (3.31), $(3.32 \mathrm{a}, \mathrm{b}),(3.19 \mathrm{~b})$, and $(3.30)$, we have, for any $L^{n} \in \mathbb{R}^{+}$, that $\left[\psi_{\alpha, \varepsilon}^{n}-L^{n} M\right]_{+} \in X_{q}$ and

$$
\begin{aligned}
a_{\alpha, \varepsilon}\left(u_{\alpha, \varepsilon}^{n}\right)\left(\left[\psi_{\alpha, \varepsilon}^{n}-L^{n} M\right]_{+},\left[\psi_{\alpha, \varepsilon}^{n}-L^{n} M\right]_{+}\right) \\
\quad=a_{\alpha, \varepsilon}\left(u_{\alpha, \varepsilon}^{n}\right)\left(\psi_{\alpha, \varepsilon}^{n},\left[\psi_{\alpha, \varepsilon}^{n}-L^{n} M\right]_{+}\right)-L^{n} a_{\alpha, \varepsilon}\left(\sim_{\alpha, \varepsilon}^{n}\right)\left(M,\left[\psi_{\alpha, \varepsilon}^{n}-L^{n} M\right]_{+}\right) \\
\quad=\ell_{\alpha, \varepsilon}^{n}\left(\left[\psi_{\alpha, \varepsilon}^{n}-L^{n} M\right]_{+}\right)-L^{n} a_{c}^{n}\left(M,\left[\psi_{\alpha, \varepsilon}^{n}-L^{n} M\right]_{+}\right) \\
\quad=\int_{\Omega \times D} W_{c}\left(\psi_{\alpha, \varepsilon}^{n-1}-L^{n} M\right)\left[\psi_{\alpha, \varepsilon}^{n}-L^{n} M\right]_{+} \mathrm{d} \underset{\sim}{q} \mathrm{~d} \underset{\sim}{x} \\
(3.70) \quad \leq \int_{\Omega \times D}\left[W_{c}\left(L^{n-1}-L^{n}\right) M\right]\left[\psi_{\alpha, \varepsilon}^{n}-L^{n} M\right]_{+} \mathrm{d}_{\sim}^{q} \mathrm{~d} \underset{\sim}{x} .
\end{aligned}
$$


Choosing $L^{n}=L^{n-1}$ yields that the right-hand side of (3.70) is zero and hence from (3.35) that $\left[\psi_{\alpha, \varepsilon}^{n}-L^{n} M\right]_{+} \equiv 0$. Thus, by induction, we have for $n=1 \rightarrow N$ that

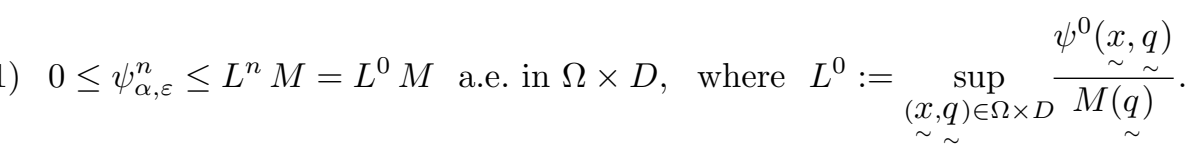

Now we introduce some definitions prior to passing to the limit $\Delta t \rightarrow 0_{+}$. Let

$$
u_{\alpha, \varepsilon}^{\Delta t}(\cdot, t):=\frac{t-t_{n-1}}{\Delta t} u_{\alpha, \varepsilon}^{n}(\cdot)+\frac{t_{n}-t}{\Delta t} u_{\alpha, \varepsilon}^{n-1}(\cdot), \quad t \in\left[t_{n-1}, t_{n}\right], \quad n \geq 1,
$$

and

$$
{\underset{\sim}{\alpha, \varepsilon}}_{\alpha, \varepsilon}^{\Delta t,+}(\cdot, t):={\underset{\sim}{u}}^{n}(\cdot), \quad{\underset{\sim}{\alpha, \varepsilon}}_{\alpha, \varepsilon}^{\Delta t,-}(\cdot, t):={\underset{\sim}{u}}^{n-1}(\cdot), \quad t \in\left(t_{n-1}, t_{n}\right], \quad n \geq 1 .
$$

We note for future reference that

$$
u_{\alpha, \varepsilon}^{\Delta t}-u_{\sim \alpha, \varepsilon}^{\Delta t, \pm}=\left(t-t_{n}^{ \pm}\right) \frac{\partial u_{\alpha, \varepsilon}^{\Delta t}}{\partial t}, \quad t \in\left(t_{n-1}, t_{n}\right), \quad n \geq 1,
$$

where $t_{n}^{+}:=t_{n}$ and $t_{n}^{-}:=t_{n-1}$. Using the above notation, and introducing analogous notation for $\left\{\psi_{\alpha, \varepsilon}^{n}\right\}_{n=0}^{N},(3.27)$ summed for $n=1 \rightarrow N$ can be restated as

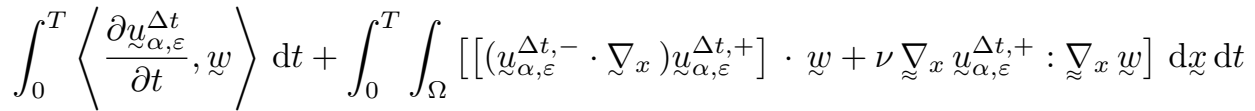

$$
\begin{aligned}
& =-k \mu \int_{0}^{T} \int_{\Omega} \underset{\approx}{C}\left(\psi_{\alpha, \varepsilon}^{\Delta t,+}\right): \underset{\approx}{\nabla}\left({\underset{\sim}{\alpha} \alpha}_{\sim}\right) \mathrm{d} x \mathrm{~d} t \quad \forall \underset{\sim}{w} \in L^{\frac{4}{4-d}}(0, T ; \underset{\sim}{V}) .
\end{aligned}
$$

Similarly, (3.31) summed for $n=1 \rightarrow N$ can be restated as

$$
\begin{aligned}
& \int_{0}^{T} \int_{\Omega \times D} W_{c} \frac{\psi_{\alpha, \varepsilon}^{\Delta t,+}-\psi_{\alpha, \varepsilon}^{\Delta t,-}}{\Delta t} \varphi \underset{\sim}{\mathrm{d}} \underset{\sim}{\mathrm{d}} x \mathrm{~d} t
\end{aligned}
$$

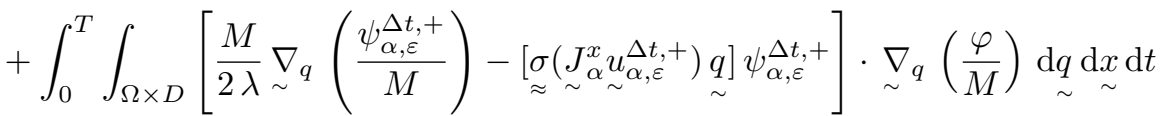

$$
\begin{aligned}
& +\int_{0}^{T} \int_{\Omega \times D}\left[\varepsilon M \underset{\sim}{\underset{\sim}{\nabla} x}\left(\frac{\psi_{\alpha, \varepsilon}^{\Delta t,+}}{M}\right)-\underset{\sim}{\sim_{\alpha, \varepsilon}^{\Delta t,+}} \psi_{\alpha, \varepsilon}^{\Delta t,+}\right] \cdot \underset{\sim}{\nabla_{x}}\left(\frac{\varphi}{M}\right) \underset{\sim}{\mathrm{d} q} \underset{\sim}{\mathrm{d}} \underset{\sim}{x} \mathrm{~d} t \\
& +\int_{0}^{T} \int_{\Omega \times D} \frac{\lambda|q|^{2}}{M}\left[A\left(\underset{\sim}{J_{\alpha}^{x}} \underset{\sim}{u_{\alpha, \varepsilon}^{\Delta t,+}}\right) \psi_{\alpha, \varepsilon}^{\Delta t,+}-A\left(\underset{\sim}{J_{\alpha}^{x}} \underset{\sim}{u_{\alpha, \varepsilon}^{\Delta t,-}}\right) \psi_{\alpha, \varepsilon}^{\Delta t,-}\right] \varphi \underset{\sim}{\mathrm{d} q} \underset{\sim}{\mathrm{d} x} \mathrm{~d} t=0 \\
& \forall \varphi \in L^{2}\left(0, T ; X_{q}\right) .
\end{aligned}
$$

We have from (3.57b) and (3.63) that

$$
\begin{gathered}
\sup _{t \in(0, T)}\left[\int_{\Omega}\left|u_{\alpha, \varepsilon}^{\Delta t(, \pm)}\right|^{2} \mathrm{~d} \underset{\sim}{x}\right]+\int_{0}^{T} \int_{\Omega} \frac{\left|u_{\alpha, \varepsilon}^{\Delta t,+}-u_{\alpha, \varepsilon}^{\Delta t,-}\right|^{2}}{\Delta t} \mathrm{~d} \underset{\sim}{x} \mathrm{~d} t \\
+\nu \int_{0}^{T} \int_{\Omega}\left|\underset{\sim}{\nabla_{x}} \underset{\sim \alpha, \varepsilon}{u t(, \pm)}\right|^{2} \mathrm{~d} \underset{\sim}{x} \mathrm{~d} t \leq C(T) .
\end{gathered}
$$


In the above, the notation ${\underset{\sim}{u, \varepsilon}}_{\alpha, \varepsilon}^{\Delta t(, \pm)}$ means ${\underset{\sim}{\alpha, \varepsilon}}_{\alpha t}^{\Delta t}$ with or without the superscripts \pm . Similarly, we have from (3.57a), (3.58), (3.68), and (3.69) that

$$
\begin{aligned}
& \sup _{t \in(0, T)}\left[\int_{\Omega \times D} \frac{\left|\psi_{\alpha, \varepsilon}^{\Delta t(, \pm)}\right|^{2}}{M} \mathrm{~d} q \underset{\sim}{\mathrm{d} x}\right]+\frac{1}{\lambda} \int_{0}^{T} \int_{\Omega \times D} M\left|\underset{\sim}{\nabla_{q}}\left(\frac{\psi_{\alpha, \varepsilon}^{\Delta t,+}}{M}\right)\right|^{2} \underset{\sim}{\mathrm{d} q} \underset{\sim}{\mathrm{d} x} \mathrm{~d} t \\
& +\varepsilon \int_{0}^{T} \int_{\Omega \times D} M\left|\underset{\sim}{\nabla_{x}}\left(\frac{\psi_{\alpha, \varepsilon}^{\Delta t,+}}{M}\right)\right|^{2} \underset{\sim}{\mathrm{d}} \underset{\sim}{\mathrm{d} x} \mathrm{~d} t+\sup _{t \in(0, T)}\left[\int_{\Omega}\left|C \underset{\approx}{\mid}\left(\psi_{\alpha, \varepsilon}^{\Delta t(, \pm)}\right)\right|^{2} \underset{\sim}{\mathrm{d} x}\right] \\
& +\int_{0}^{T}\left[\int_{\Omega \times D} W_{c} \frac{\left|\psi_{\alpha, \varepsilon}^{\Delta t,+}-\psi_{\alpha, \varepsilon}^{\Delta t,-}\right|^{2}}{\Delta t} \mathrm{~d} \underset{\sim}{q} \mathrm{~d} x+\left\|\underset{\sim}{\underset{\sim}{\gamma} \underset{\sim}{\partial u_{\alpha, \varepsilon}^{\Delta t}}} \frac{\|^{\frac{4}{d}}}{\partial t}\right\|_{H^{1}(\Omega)}\right] \mathrm{d} t \\
& \leq\left\{\begin{array}{lll}
C(T) & \text { if } & \underset{\sim}{ }(\cdot)=\underset{\sim}{\omega}(\cdot) \\
C(\alpha, T) & \text { if } & g(\cdot)=\nabla_{x}(\cdot) \text { and } D \text { is bounded }
\end{array}\right.
\end{aligned}
$$

We are now in a position to prove the following convergence result.

Lemma 3.3. There exists a subsequence of $\left\{u_{\alpha, \varepsilon}^{\Delta t}, \psi_{\alpha, \varepsilon}^{\Delta t}\right\}_{\Delta t}$, and functions ${\underset{\sim}{\alpha, \varepsilon}}_{\alpha} \in$ $L^{\infty}\left(0, T ; \underset{\sim}{L^{2}}(\Omega)\right) \cap L^{2}(0, T ; \underset{\sim}{V}) \cap W^{1, \frac{4}{d}}\left(0, T ; \underset{\sim}{V^{\prime}}\right)$ and $\psi_{\alpha, \varepsilon} \in L^{2}\left(0, T ; X^{+}\right)$with $M^{-\frac{1}{2}}$ $\psi_{\alpha, \varepsilon} \in L^{\infty}\left(0, T ; L^{2}(\Omega \times D)\right)$, such that, as $\Delta t \rightarrow 0$,

$$
\begin{aligned}
& \frac{\psi_{\alpha, \varepsilon}^{\Delta t(, \pm)}}{M^{\frac{1}{2}}} \rightarrow \frac{\psi_{\alpha, \varepsilon}}{M^{\frac{1}{2}}} \quad \text { weak }^{*} \text { in } L^{\infty}\left(0, T ; L^{2}(\Omega \times D)\right), \\
& M^{\frac{1}{2}} \underset{\sim}{\nabla_{q}}\left(\frac{\psi_{\alpha, \varepsilon}^{\Delta t,+}}{M}\right) \rightarrow M^{\frac{1}{2}} \underset{\sim}{\nabla_{q}}\left(\frac{\psi_{\alpha, \varepsilon}}{M}\right) \quad \text { weakly in } L^{2}\left(0, T ; \underset{\sim}{L^{2}}(\Omega \times D)\right) \text {, } \\
& M^{\frac{1}{2}} \underset{\sim}{\nabla_{x}}\left(\frac{\psi_{\alpha, \varepsilon}^{\Delta t,+}}{M}\right) \rightarrow M^{\frac{1}{2}} \underset{\sim}{\nabla_{x}}\left(\frac{\psi_{\alpha, \varepsilon}}{M}\right) \quad \text { weakly in } L^{2}\left(0, T ; \underset{\sim}{\left.L^{2}(\Omega \times D)\right),}\right. \\
& \underset{\approx}{C}\left(\psi_{\alpha, \varepsilon}^{\Delta t(, \pm)}\right) \rightarrow \underset{\approx}{C}\left(\psi_{\alpha, \varepsilon}\right) \quad \text { weak }{ }^{*} \text { in } L^{\infty}\left(0, T ; \underset{\approx}{L^{2}}(\Omega)\right),
\end{aligned}
$$

and

$$
\begin{aligned}
& {\underset{\sim}{u, \varepsilon}}_{\alpha, \varepsilon}^{\Delta t(, \pm)} \rightarrow{\underset{\sim}{\alpha, \varepsilon}}_{\alpha, \varepsilon} \quad \text { weak } \text { in }^{\infty}\left(0, T ; \underset{\sim}{L^{2}}(\Omega)\right), \\
& u_{\sim \alpha, \varepsilon}^{\Delta t(, \pm)} \rightarrow u_{\alpha, \varepsilon} \quad \text { weakly in } L^{2}(0, T ; \underset{\sim}{V}) \text {, } \\
& \underset{\sim}{S_{\gamma}} \frac{\partial u_{\alpha, \varepsilon}^{\Delta t}}{\partial t} \rightarrow \underset{\sim}{S_{\gamma}} \frac{\partial u_{\alpha, \varepsilon}}{\partial t} \quad \text { weakly in } L^{\frac{4}{d}}(0, T ; V) \text {, } \\
& u_{\alpha, \varepsilon}^{\Delta t(, \pm)} \rightarrow u_{\alpha, \varepsilon} \quad \text { strongly in } L^{2}\left(0, T ; \sim_{\sim}^{r}(\Omega),\right. \\
& J_{\alpha}^{x} u_{\alpha, \varepsilon}^{\Delta t(, \pm)} \rightarrow J_{\alpha}^{x} u_{\sim \alpha, \varepsilon} \quad \text { strongly in } L^{\infty}\left(0, T ; W^{1, \infty}(\Omega)\right) \text {, }
\end{aligned}
$$

where $r \in[1, \infty)$ if $d=2$ and $r \in[1,6)$ if $d=3$.

Proof. The result (3.78a) follows immediately from the bounds on the first and third terms on the left-hand side of (3.77), noting (3.36) and the notation (3.72a,b). 
It follows immediately from the bound on the second term on the left-hand side of (3.77) that (3.78b) holds for some limit $\underset{\sim}{g} \in L^{2}\left(0, T ; \underset{\sim}{L^{2}}(\Omega \times D)\right)$, which we need to identify. However, for any $\eta \in L^{2}\left(0, T ; \underset{\sim}{C_{0}^{\infty}}(\Omega \times D)\right)$, it follows from (2.9) and the compact support of $\underset{\sim}{\eta}$ on $D$ that $\left[\underset{\sim}{\nabla} \cdot\left(M^{\frac{1}{2}} \underset{\sim}{\eta}\right)\right] / M^{\frac{1}{2}} \in L^{2}\left(0, T ; L^{2}(\Omega \times D)\right)$, and hence the above convergence implies, noting $(3.78 \mathrm{a})$, that

$$
\begin{aligned}
\int_{0}^{T} \int_{\Omega \times D} \underset{\sim}{g} \cdot \underset{\sim}{\eta} \mathrm{d} \underset{\sim}{q} \mathrm{~d} \underset{\sim}{x} \mathrm{~d} t & \leftarrow-\int_{0}^{T} \int_{\Omega \times D} \frac{\psi_{\alpha, \varepsilon}^{\Delta t,+}}{M^{\frac{1}{2}}} \frac{\nabla_{q} \cdot\left(M^{\frac{1}{2}} \underset{\sim}{\eta}\right)}{M^{\frac{1}{2}}} \mathrm{~d} \underset{\sim}{q} \mathrm{~d} \underset{\sim}{x} \mathrm{~d} t \\
& \rightarrow-\int_{0}^{T} \int_{\Omega \times D} \frac{\psi_{\alpha, \varepsilon}}{M^{\frac{1}{2}}} \frac{\underset{q}{\nabla} \cdot\left(M^{\frac{1}{2}} \underset{\sim}{\eta}\right)}{M^{\frac{1}{2}}} \mathrm{~d} \underset{\sim}{q} \mathrm{~d} \underset{\sim}{x} \mathrm{~d} t
\end{aligned}
$$

as $\Delta t \rightarrow 0$. Hence the desired result (3.78b) follows from (3.80), noting the denseness of $C_{0}^{\infty}(\Omega \times D)$ in $L^{2}(\Omega \times D)$. A similar argument also proves $(3.78 \mathrm{c})$. The desired result (3.78d) follows immediately from (3.78a) and (2.4a). Finally, the nonnegativity of $\psi_{\alpha, \varepsilon}$ follows from that of $\psi_{\alpha, \varepsilon}^{\Delta t(, \pm)}$; recall Lemma 3.2.

The results $(3.79 \mathrm{a}-\mathrm{c})$ follow immediately from the bounds (3.76) and the bound on ${\underset{\sim}{\alpha, \varepsilon}}_{\alpha t}^{\Delta t}$ in (3.77). The strong convergence result $(3.79 \mathrm{~d})$ for $u_{\alpha, \varepsilon}^{\Delta t}$ follows immediately from $(3.79 \mathrm{a}-\mathrm{c}),(3.3)$, and a standard compactness result, noting that $\underset{\sim}{V} \subset \underset{\sim}{H}{ }_{0}^{1}(\Omega)$ is compactly embedded in $\underset{\sim}{L^{r}}(\Omega)$ for the stated values of $r$. We now prove $(3.79 \mathrm{~d})$ for $\underset{\sim \alpha, \varepsilon}{u t, \pm}$. First, we obtain from the bound on the second term on the left-hand side of (3.76) and from (3.73) that

$$
\left\|{\underset{\sim}{u, \varepsilon}}_{\alpha, \varepsilon}^{\Delta t}-\underset{\sim \alpha, \varepsilon}{u t}\right\|_{L^{2}\left(0, T, L^{2}(\Omega)\right)}^{2 t,} \leq C \Delta t
$$

Second, we note from Sobolev embedding that, for all $\eta \in L^{2}\left(0, T ; H^{1}(\Omega)\right)$,

$$
\|\eta\|_{L^{2}\left(0, T ; L^{r}(\Omega)\right)} \leq C\|\eta\|_{L^{2}\left(0, T ; L^{2}(\Omega)\right)}^{1-\beta}\|\eta\|_{L^{2}\left(0, T ; H^{1}(\Omega)\right)}^{\beta}
$$

for any $r \in[2, \infty)$ if $d=2$ or any $r \in[2,6)$ if $d=3$, where $\beta=d\left(\frac{1}{2}-\frac{1}{r}\right) \in[0,1)$. Hence, combining (3.81), (3.82), and (3.79d) for ${\underset{\sim}{\alpha, \varepsilon}}_{\alpha t}^{\Delta t}$ yields $(3.79 \mathrm{~d})$ for $\underset{\sim \alpha, \varepsilon}{u t, \pm}$. Finally, the desired result $(3.79 \mathrm{e})$ follows immediately from $(3.9 \mathrm{~b})$ and $(3.79 \mathrm{~d})$.

Similarly to $(3.82)$, we have, for any $r \in[2, \infty)$ if $d=2$ or any $r \in[2,6]$ if $d=3$, that

$$
\|\eta\|_{L^{\frac{2}{\beta}}\left(0, T ; L^{r}(\Omega)\right)} \leq C\|\eta\|_{L^{2}\left(0, T ; H^{1}(\Omega)\right)} \quad \text { if } \eta \in L^{\infty}\left(0, T ; L^{2}(\Omega)\right)
$$

where $\beta=d\left(\frac{1}{2}-\frac{1}{r}\right) \in[0,1]$. It follows from $(3.79 \mathrm{a}-\mathrm{d}),(3.78 \mathrm{~d}),(3.29),(3.83)$, and (3.2) that we may pass to the limit, $\Delta t \rightarrow 0$, in (3.74) to obtain that ${\underset{\sim}{\alpha, \varepsilon}}_{\epsilon} \in$ $L^{\infty}\left(0, T ; \underset{\sim}{L^{2}}(\Omega)\right) \cap L^{2}(0, T ; \underset{\sim}{V}) \cap W^{1, \frac{4}{d}}\left(0, T ; \underset{\sim}{V^{\prime}}\right)$ and $\underset{\approx}{C}\left(\psi_{\alpha, \varepsilon}\right) \in L^{\infty}\left(0, T ; \underset{\approx}{L^{2}}(\Omega)\right)$ satisfy (3.21a). It also follows from $(3.23)$ that $\underset{\sim}{u} \alpha, \varepsilon(\cdot, 0)=\underset{\sim}{u_{0}}(\cdot)$ in the required sense.

As we have no control of the time derivative $\psi_{\alpha, \varepsilon}^{\Delta t}$, in order to pass to the $\Delta t \rightarrow 0$ limit in (3.75) this derivative has to be transferred to the test function. We have for 
any fixed $\varphi \in C_{0}^{\infty}((-T, T) ; \mathcal{K})$ that

$$
\begin{aligned}
& \int_{0}^{T} \int_{\Omega \times D} W_{c} \frac{\psi_{\alpha, \varepsilon}^{\Delta t,+}(\underset{\sim}{x}, \underset{\sim}{q}, t)-\psi_{\alpha, \varepsilon}^{\Delta t,-}(\underset{\sim}{x}, \underset{\sim}{q}, t)}{\Delta t} \varphi(\underset{\sim}{x}, \underset{\sim}{q}, t) \mathrm{d} \underset{\sim}{q} \mathrm{~d} \underset{\sim}{x} \mathrm{~d} t \\
&=-\int_{0}^{T} \int_{\Omega \times D} W_{c} \psi_{\alpha, \varepsilon}^{\Delta t,-}(\underset{\sim}{x}, \underset{\sim}{q}, t) \frac{\varphi(\underset{\sim}{x}, \underset{\sim}{q}, t)-\varphi(\underset{\sim}{x}, \underset{\sim}{q}, t-\Delta t)}{\Delta t} \mathrm{~d} \underset{\sim}{q} \mathrm{~d} \underset{\sim}{x} \mathrm{~d} t \\
& \quad-\int_{\Omega \times D} W_{c} \psi^{0}(\underset{\sim}{x}, \underset{\sim}{q})\left(\frac{1}{\Delta t} \int_{0}^{t_{1}} \varphi(\underbrace{x}_{\sim}, \underset{\sim}{q}, t-\Delta t) \mathrm{d} t\right) \mathrm{d} \underset{\sim}{q} \mathrm{~d} \underset{\sim}{x} .
\end{aligned}
$$

It follows for all $\varphi \in C_{0}^{\infty}((-T, T) ; \mathcal{K})$ and for all $\left.\underset{\sim}{\underset{\sim}{x}} \underset{\sim}{q}, t\right) \in \Omega \times D \times(0, T)$ that

$$
\frac{\varphi(\underset{\sim}{x}, \underset{\sim}{q}, t)-\varphi(\underset{\sim}{x}, \underset{\sim}{q}, t-\Delta t)}{\Delta t}=\frac{\partial \varphi}{\partial t}(\underset{\sim}{x} \underset{\sim}{q}, t)+R_{\Delta t}(\varphi)(\underset{\sim}{x} \underset{\sim}{q}, t),
$$

where

$$
\left|R_{\Delta t}(\varphi)(\underset{\sim}{x} \underset{\sim}{q}, t)\right| \leq \frac{\Delta t}{2} \underset{(\underset{\sim}{x}, \underset{\sim}{q}, t) \in \max _{\Omega} \times D \times[-T, T]}{ }\left|\frac{\partial^{2} \varphi}{\partial t^{2}}(\underset{\sim}{x}, \underset{\sim}{q}, t)\right| .
$$

Hence, combining (3.75), (3.84), and (3.85), we have for any fixed $\varphi \in C_{0}^{\infty}((-T, T)$; $\mathcal{K})$ that

$$
\begin{aligned}
& -\int_{0}^{T} \int_{\Omega \times D} W_{c} \psi_{\alpha, \varepsilon}^{\Delta t,-}\left[\frac{\partial \varphi}{\partial t}+R_{\Delta t}(\varphi)\right] \mathrm{d} \underset{\sim}{\mathrm{d}} \mathrm{d} x \mathrm{~d} t \\
& -\int_{\Omega \times D} W_{c} \psi^{0}(\underset{\sim}{x} \underset{\sim}{q})\left(\frac{1}{\Delta t} \int_{0}^{t_{1}} \varphi(\underset{\sim}{x}, \underset{\sim}{q}, t-\Delta t) \mathrm{d} t\right) \mathrm{d} q \underset{\sim}{\mathrm{d}} \underset{\sim}{x} \\
& +\int_{0}^{T} \int_{\Omega \times D}\left[\frac{M}{2 \lambda} \nabla_{\sim}\left(\frac{\psi_{\alpha, \varepsilon}^{\Delta t,+}}{M}\right)-\left[\underset{\sim}{\sigma}\left({\underset{\sim}{\alpha}}_{\alpha}^{x} u_{\alpha, \varepsilon}^{\Delta t,+}\right) \underset{\sim}{q}\right] \psi_{\alpha, \varepsilon}^{\Delta t,+}\right] \cdot \nabla_{q}\left(\frac{\varphi}{M}\right) \mathrm{d} q \underset{\sim}{\mathrm{d} x} \mathrm{~d} t \\
& +\int_{0}^{T} \int_{\Omega \times D}\left[\varepsilon M \underset{\sim}{\nabla_{x}}\left(\frac{\psi_{\alpha, \varepsilon}^{\Delta t,+}}{M}\right)-{\underset{\sim}{\alpha, \varepsilon}}_{\alpha t,+}^{\Delta t} \psi_{\alpha, \varepsilon}^{\Delta t,+}\right] \cdot \nabla_{x}\left(\frac{\varphi}{M}\right) \mathrm{d} \underset{\sim}{q} \mathrm{~d} \underset{\sim}{x} \mathrm{~d} t \\
& +\int_{0}^{T} \int_{\Omega \times D} \frac{\lambda|q|^{2}}{M}\left[A\left(J_{\alpha}^{x} \underset{\sim \alpha, \varepsilon}{\Delta t,+}\right) \psi_{\alpha, \varepsilon}^{\Delta t,+}-A\left(J_{\alpha}^{x}{\underset{\sim}{\alpha, \varepsilon}}_{\alpha,-}^{\Delta t,-}\right) \psi_{\alpha, \varepsilon}^{\Delta t,-}\right] \varphi \underset{\sim}{q} \mathrm{~d} \underset{\sim}{x} \mathrm{~d} t=0 .
\end{aligned}
$$

Now, similarly to (3.84), we have from (3.85), (3.77), (3.25), (3.9b), and (3.76) for any $\varphi \in C_{0}^{\infty}((-T, T) ; \mathcal{K})$ that

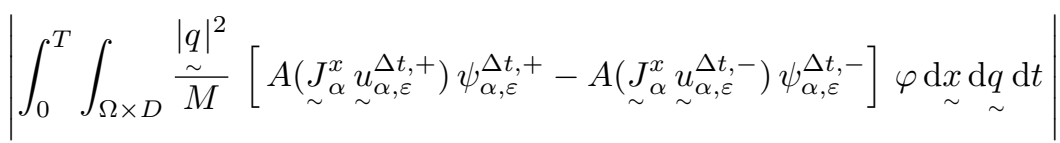

$$
\begin{aligned}
& =\Delta t \mid \int_{0}^{T} \int_{\Omega \times D} \frac{|q|^{2}}{\stackrel{\sim}{M}} A\left(J_{\sim}^{x} \underset{\sim}{u_{\alpha, \varepsilon}^{\Delta t,-}}\right) \psi_{\alpha, \varepsilon}^{\Delta t,-}\left[\frac{\partial \varphi}{\partial t}+R_{\Delta t}(\varphi)\right] \underset{\sim}{\mathrm{d} q} \underset{\sim}{\mathrm{d} x} \mathrm{~d} t
\end{aligned}
$$

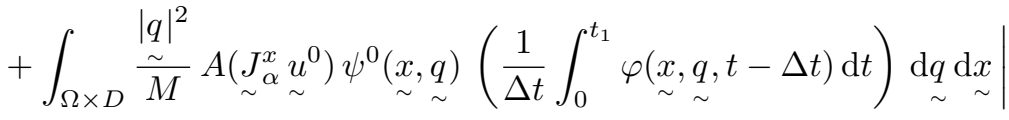

$$
\begin{aligned}
& \leq C(\varphi) \Delta t \int_{0}^{T} A\left(\underset{\sim}{J_{\alpha}^{x}} \underset{\sim}{u, \varepsilon} \Delta t,-\right) \mathrm{d} t \leq C(\varphi) \Delta t .
\end{aligned}
$$


It follows from $(3.78 \mathrm{a}-\mathrm{c}),(3.79 \mathrm{~d}, \mathrm{e}),(3.85),(3.87),(3.36)$, and (3.23) that we may pass to the limit $\Delta t \rightarrow 0$ in (3.86) to obtain that $\psi_{\alpha, \varepsilon} \in L^{2}(0, T ; X)$ with $M^{-\frac{1}{2}} \psi_{\alpha, \varepsilon} \in$ $L^{\infty}\left(0, T ; L^{2}(\Omega \times D)\right)$ and ${\underset{\sim}{\alpha, \varepsilon}}_{\alpha} \in L^{2}(0, T ; \underset{\sim}{V})$ satisfy

$$
\begin{aligned}
& -\int_{0}^{T} \int_{\Omega \times D} \frac{\psi_{\alpha, \varepsilon}}{M} \frac{\partial \varphi}{\partial t} \mathrm{~d} \underset{\sim}{q} \mathrm{~d} \underset{\sim}{\mathrm{d}} \mathrm{d} t-\int_{\Omega \times D} \frac{\psi_{0}(\cdot, \cdot)}{M} \varphi(\cdot, \cdot, 0) \mathrm{d} \underset{\sim}{\mathrm{d}} \mathrm{d} \underset{\sim}{M} \\
& +\int_{0}^{T} \int_{\Omega \times D}\left[\frac{M}{2 \lambda} \nabla_{q}\left(\frac{\psi_{\alpha, \varepsilon}}{M}\right)-\left[\underset{\sim}{\sigma}\left(J_{\alpha}^{x} \underset{\sim}{u} \alpha, \varepsilon\right) \underset{\sim}{q}\right] \psi_{\alpha, \varepsilon}\right] \cdot \underset{\sim}{\nabla_{q}}\left(\frac{\varphi}{M}\right) \mathrm{d} \underset{\sim}{q} \mathrm{~d} \underset{\sim}{x} \mathrm{~d} t \\
& +\int_{0}^{T} \int_{\Omega \times D}\left[\varepsilon M \underset{\sim}{\nabla_{x}}\left(\frac{\psi_{\alpha, \varepsilon}}{M}\right)-{\underset{\sim}{\alpha, \varepsilon}}_{\alpha, \varepsilon}\right] \cdot \underset{\sim}{\nabla_{x}}\left(\frac{\varphi}{M}\right) \underset{\sim}{\mathrm{d} q} \mathrm{~d} \underset{\sim}{\mathrm{d}} \mathrm{d} t=0 \\
& \forall \varphi \in C_{0}^{\infty}((-T, T) ; \mathcal{K}) .
\end{aligned}
$$

Noting (3.33), (3.83), and that $C_{0}^{\infty}((-T, T) ; \mathcal{K})$ is a dense subset of $\mathcal{X}$, recall (3.22), it follows that (3.88) remains true for all $\varphi \in \mathcal{X}$. Hence we have proved existence of a global weak solution of $\left(\mathrm{P}_{\alpha, \varepsilon}\right),(3.21 \mathrm{a}, \mathrm{b})$. Moreover, it follows from (3.76), (3.77), $(3.78 \mathrm{a}-\mathrm{d})$, and $(3.79 \mathrm{a}-\mathrm{c})$ that

$$
\sup _{t \in(0, T)}\left[\int_{\Omega}\left|u_{\alpha, \varepsilon}\right|^{2} \mathrm{~d} x\right]+\nu \int_{0}^{T} \int_{\Omega}\left|\nabla_{x} u_{\sim \alpha, \varepsilon}\right|^{2} \mathrm{~d} x \mathrm{~d} t \leq C(T),
$$

$$
\begin{aligned}
& \sup _{t \in(0, T)}\left[\int_{\Omega \times D} \frac{\left|\psi_{\alpha, \varepsilon}\right|^{2}}{M} \mathrm{~d} \underset{\sim}{\mathrm{d}} \mathrm{d} x\right]+\frac{1}{\lambda} \int_{0}^{T} \int_{\Omega \times D} M\left|\nabla_{q}\left(\frac{\psi_{\alpha, \varepsilon}}{M}\right)\right|^{2} \mathrm{~d} \underset{\sim}{\mathrm{d}} \mathrm{d} \underset{\sim}{\mathrm{d} t} t \\
& +\varepsilon \int_{0}^{T} \int_{\Omega \times D} M\left|\nabla_{x}\left(\frac{\psi_{\alpha, \varepsilon}}{M}\right)\right|^{2} \mathrm{~d} \underset{\sim}{q} \mathrm{~d} \underset{\sim}{\mathrm{d} t}+\sup _{t \in(0, T)}\left[\int_{\Omega}\left|\underset{\sim}{C}\left(\psi_{\alpha, \varepsilon}\right)\right|^{2} \mathrm{~d} x\right] \\
& +\int_{0}^{T}\left\|{\underset{\sim}{S} \gamma}_{\gamma} \frac{\partial u_{\alpha, \varepsilon}}{\partial t}\right\|_{H^{1}(\Omega)}^{\frac{4}{d}} \mathrm{~d} t \leq\left\{\begin{array}{lll}
C(T) & \text { if } & \underset{\sigma}{(}(\cdot)=\underset{\sim}{(}(\cdot) \\
C(\alpha, T) & \text { if } & \underset{\sim}{g}(\cdot)=\approx_{x}(\cdot) \text { and } D \text { is bounded. }
\end{array}\right.
\end{aligned}
$$

Remark 3.4. In the corotational case, if $L^{0}$ is finite in (3.71), then we have a uniform $L^{\infty}\left(0, T ; L^{\infty}(\Omega \times D)\right)$ bound on $M^{-1} \psi_{\alpha, \varepsilon}^{\Delta(, \pm)}$. Moreover, it is then easily established that the limit $M^{-1} \psi_{\alpha, \varepsilon} \in L^{\infty}\left(0, T ; L^{\infty}(\Omega \times D)\right)$ with $\psi_{\alpha, \varepsilon} \geq 0$ a.e. on $\Omega \times D \times(0, T)$.

Remark 3.5. The argument presented above for noncorotational FENE-type models breaks down for noncorotational Hookean models, since in the transition from bound (3.65) to (3.66) we exploit the fact that $D$ is bounded. The difficulty could be overcome if one could obtain a maximum principle on $\psi^{n}$ along the lines of (3.70). Unfortunately, in the case of $D=\mathbb{R}^{d}$ this does not appear to be readily achievable. Having said this, our main focus of interest in the present article is FENE-type microscopic-macroscopic models for diluted polymers where $D$ is a bounded open ball in $\mathbb{R}^{d}$ : the fact that in Hookean-type models the domain $D$ is equal to the whole of $\mathbb{R}^{d}$ stems from the physically unrealistic modelling assumption that the length $|\underset{\sim}{q}|$ of the elongation vector $q \in D$ of a polymer chain may be arbitrarily large.

Remark 3.6. Since the test functions in $\underset{\sim}{V}$ are divergence-free, the pressure has been eliminated in $(3.21 \mathrm{a}, \mathrm{b})$; it can be recovered in a very weak sense following the 
same procedure as for the incompressible Navier-Stokes equations discussed on p. 208 in Temam [28]; i.e., one obtains that $\int_{0}^{t} p_{\alpha, \varepsilon}(\cdot, s) \mathrm{d} s \in C\left([0, T] ; L^{2}(\Omega)\right)$.

Remark 3.7. It is a simple matter to adapt the proofs above to show that the main results above remain true for $\left(\mathrm{P}_{\alpha, \varepsilon}\right)$ if we replace the smoothing procedure $J_{\alpha}^{x}$ by ${\underset{\sim}{\alpha}}_{\alpha}$ (including the definition of $\underset{\sim}{I}$ in (3.20)). The key results, (3.8b) and (3.9a,b), that we exploit for $J_{\alpha}^{x}$ in the above, are now replaced by (3.4b) and (3.6b) for $S_{\alpha}$. Unfortunately, we require $\partial \Omega \in C^{2}$ for (3.6b), as opposed to $\partial \Omega \in C^{0,1}$ for (3.9b). Hence we prefer, in general, $J_{\alpha}^{x}$ over ${\underset{\sim}{\alpha}}_{\alpha}$. However, ${\underset{\sim}{\alpha}}_{\alpha}$ does have one key advantage over $\underset{\sim}{J}$ in that $\underset{\sim}{S} \underset{\sim}{v} \in \underset{\sim}{H}{ }_{0}^{1}(\Omega)$ if $\underset{\sim}{v} \in \underset{\sim}{H}{ }_{0}^{1}(\Omega)$.

3.2. Existence for $\left(\mathbf{P}_{\boldsymbol{\alpha}}\right)$. As the bounds $(3.89 \mathrm{a}, \mathrm{b})$ are independent of the parameter $\varepsilon$, it follows immediately, similarly to $(3.78 \mathrm{a}-\mathrm{d}),(3.79 \mathrm{a}-\mathrm{d})$, and $(3.89 \mathrm{a}, \mathrm{b})$, that the following lemma holds.

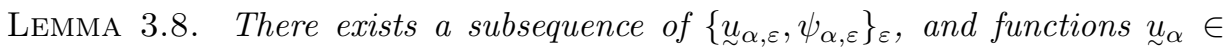
$L^{\infty}\left(0, T ; L_{\sim}^{2}(\Omega)\right) \cap L^{2}(0, T ; \underset{\sim}{V}) \cap W^{1, \frac{4}{d}}\left(0, T ; V_{\sim}^{\prime}\right)$ and $\psi_{\alpha} \in L^{2}\left(0, T ; X^{0,+}\right)$ with $M^{-\frac{1}{2}} \psi_{\alpha}$ $\in L^{\infty}\left(0, T ; L^{2}(\Omega \times D)\right)$, such that, as $\varepsilon \rightarrow 0$,

$$
\begin{aligned}
M^{\frac{1}{2}} \underset{\sim}{\nabla_{q}}\left(\frac{\psi_{\alpha, \varepsilon}}{M}\right) & \rightarrow M^{\frac{1}{2}} \underset{\sim}{\nabla_{q}}\left(\frac{\psi_{\alpha}}{M}\right) & \text { weakly in } L^{2}\left(0, T ; \underset{\sim}{L^{2}}(\Omega \times D)\right), \\
\varepsilon M^{\frac{1}{2}} \underset{\sim}{\nabla_{x}}\left(\frac{\psi_{\alpha, \varepsilon}}{M}\right) & \rightarrow 0 & \text { weakly in } L^{2}\left(0, T ; \underset{\sim}{\left.L^{2}(\Omega \times D)\right),}\right. \\
\underset{\approx}{C}\left(\psi_{\alpha, \varepsilon}\right) & \rightarrow \underset{\approx}{C}\left(\psi_{\alpha}\right) & \text { weak }^{*} \text { in } L^{\infty}\left(0, T \underset{\approx}{T} \underset{\sim}{\left.L^{2}(\Omega)\right),}\right.
\end{aligned}
$$

and

$$
\begin{aligned}
& {\underset{\sim}{\alpha, \varepsilon}}_{\alpha} \rightarrow{\underset{\sim}{\alpha}}_{\alpha} \quad \text { weak }{ }^{*} \text { in } L^{\infty}\left(0, T ; \underset{\sim}{L^{2}}(\Omega)\right), \\
& \stackrel{u}{\alpha, \varepsilon}_{\alpha} \rightarrow u_{\alpha} \quad \text { weakly in } L^{2}(0, T ; V), \\
& \underset{\sim}{S_{\gamma}} \frac{\partial \underline{u}_{\alpha, \varepsilon}}{\partial t} \rightarrow \underset{\sim}{S_{\gamma}} \frac{\partial u_{\alpha}}{\partial t} \quad \text { weakly in } L^{\frac{4}{d}}(0, T ; \underset{\sim}{V}), \\
& {\underset{\sim}{u}, \varepsilon}_{\alpha} \rightarrow{\underset{\sim}{u}}_{\alpha} \quad \text { strongly in } L^{2}\left(0, T ; \underset{\sim}{L^{r}}(\Omega),\right.
\end{aligned}
$$

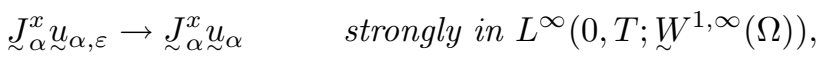

where $r \in[1, \infty)$ if $d=2$ and $r \in[1,6)$ if $d=3$. 
In addition, we have that

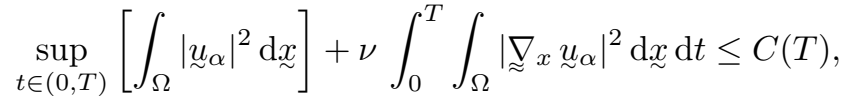

$$
\begin{aligned}
& \sup _{t \in(0, T)}\left[\int_{\Omega \times D} \frac{\left|\psi_{\alpha}\right|^{2}}{M} \mathrm{~d} q \underset{\sim}{\mathrm{d}} \underset{\sim}{x}\right]+\frac{1}{\lambda} \int_{0}^{T} \int_{\Omega \times D} M\left|\nabla_{q}\left(\frac{\psi_{\alpha}}{M}\right)\right|^{2} \mathrm{~d} \underset{\sim}{q} \mathrm{~d} \underset{\sim}{\mathrm{d}} \mathrm{d} t
\end{aligned}
$$

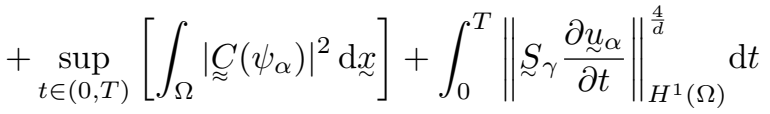

$$
\begin{aligned}
& \leq \begin{cases}C(T) & \text { if } \quad \underset{\sim}{g}(\cdot)=\underset{\sim}{\omega}(\cdot), \\
C(\alpha, T) & \text { if } \quad \underset{\sim}{g}(\cdot)=\bigotimes_{x}(\cdot) \text { and } D \text { is bounded. }\end{cases}
\end{aligned}
$$

Therefore in both the corotational and the noncorotational cases we can then pass to limit $\varepsilon \rightarrow 0$ in $\left(\mathrm{P}_{\alpha, \varepsilon}\right)$ to obtain existence of a weak solution to the following problem for a given $\alpha \in(0,1]$ :

$\left(\mathrm{P}_{\alpha}\right)$ Find ${\underset{\sim}{\alpha}}_{\alpha} \in L^{\infty}\left(0, T ; \underset{\sim}{L^{2}}(\Omega)\right) \cap L^{2}(0, T ; \underset{\sim}{V}) \cap W^{1, \frac{4}{d}}\left(0, T ; \underset{\sim}{V^{\prime}}\right)$ and $\psi_{\alpha} \in L^{2}(0, T ;$ $\left.X^{0}\right)$, with $\sim_{\alpha}^{x} u_{\alpha} \in L^{\infty}\left(0, T ; \underset{\sim}{W^{1, \infty}}(\Omega)\right), M^{-\frac{1}{2}} \psi_{\alpha} \in L^{\infty}\left(0, T ; L^{2}(\Omega \times D)\right)$ and $\underset{\approx}{C}\left(\psi_{\alpha}\right) \in$ $L^{\infty}\left(0, T ; \underset{\sim}{L^{2}}(\Omega)\right)$, such that $\underset{\sim}{\sim} \alpha(\cdot, 0)=\underset{\sim}{u_{0}}(\cdot)$ and

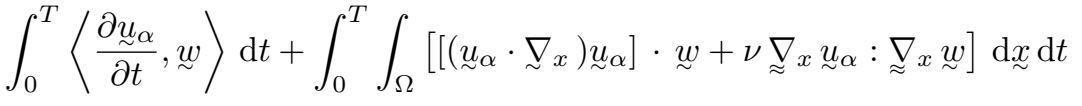

$$
\begin{aligned}
& =-k \mu \int_{0}^{T} \int_{\Omega} \underset{\approx}{C}\left(\psi_{\alpha}\right): \nabla_{x}(\underset{\sim}{I} \alpha \underset{\sim}{w}) \mathrm{d} x \mathrm{~d} t \quad \forall \underset{\sim}{w} \in L^{\frac{4}{4-d}}(0, T ; V), \\
& -\int_{0}^{T} \int_{\Omega \times D} \frac{\psi_{\alpha}}{M} \frac{\partial \varphi}{\partial t} \mathrm{~d} \underset{\sim}{\mathrm{d}} \mathrm{d} x \mathrm{~d} t-\int_{\Omega \times D} \frac{\psi_{0}(\cdot, \cdot)}{M} \varphi(\cdot, \cdot, 0) \mathrm{d} \underset{\sim}{\mathrm{d}} \mathrm{d} x \\
& +\int_{0}^{T} \int_{\Omega \times D}\left[\frac{M}{2 \lambda} \nabla_{q}\left(\frac{\psi_{\alpha}}{M}\right)-\left[\underset{\sim}{\sigma}\left(J_{\alpha}^{x} u_{\alpha}\right) \underset{\sim}{q}\right] \psi_{\alpha}\right] \cdot \underset{\sim}{\nabla_{q}}\left(\frac{\varphi}{M}\right) \mathrm{d} \underset{\sim}{\mathrm{d}} \mathrm{d} \underset{\sim}{\mathrm{d}} t \\
& -\int_{0}^{T} \int_{\Omega \times D} \underset{\sim}{u_{\alpha}} \psi_{\alpha} \cdot \underset{\sim}{\nabla_{x}}\left(\frac{\varphi}{M}\right) \mathrm{d} \underset{\sim}{\mathrm{d}} \mathrm{d} \underset{\sim}{x} \mathrm{~d} t=0 \quad \forall \varphi \in \mathcal{X}^{0},
\end{aligned}
$$

where $\mathcal{X}^{0}$ is the completion of $C_{0}^{\infty}\left((-T, T) ; \mathcal{K}^{0}\right)$ in the norm $\|\cdot\|_{\mathcal{X}^{0}}$ defined by

$$
\begin{aligned}
\|\varphi\|_{\mathcal{X}^{0}}:= & \|\varphi\|_{L^{2}\left(0, T ; X_{q}^{0}\right)}+\left\||\underset{\sim}{q}| M^{\frac{1}{2}} \nabla_{q}\left(\frac{\varphi}{M}\right)\right\|_{L^{1}\left(0, T ; L^{2}(\Omega \times D)\right)} \\
& +\left\|M^{-\frac{1}{2}} \nabla_{x} \varphi\right\|_{L^{2}\left(0, T ; L^{r}\left(\Omega ; L^{2}(D)\right)\right)}+\left\|M^{-\frac{1}{2}} \frac{\partial \varphi}{\partial t}\right\|_{L^{1}\left(0 ; T ; L^{2}(\Omega \times D)\right)}
\end{aligned}
$$

with $r>2$ if $d=2$ or $r=3$ if $d=3$. This, in particular, implies that each $\varphi \in \mathcal{X}^{0}$ satisfies $\varphi(\cdot, \cdot, T)=0$.

Remark 3.9. In view of Remark 3.4, in the corotational case if $L^{0}$ is finite in (3.71), then one can show that $M^{-1} \psi_{\alpha} \in L^{\infty}\left(0, T ; L^{\infty}(\Omega \times D)\right)$ with $\psi_{\alpha} \geq 0$ a.e. on $\Omega \times D \times(0, T)$. Hence the norm $\|\cdot\|_{\mathcal{X}^{0}}$ in $(3.94)$ can be relaxed to the weaker 
norm $\|\varphi\|_{L^{2}\left(0, T ; X_{q}^{0}\right)}+\left\||\underset{\sim}{q}| M{\underset{\sim}{\nabla}}_{q}\left(\frac{\varphi}{M}\right)\right\|_{L^{1}\left(0, T ; L^{1}(\Omega \times D)\right)}+\left\|\nabla_{x} \varphi\right\|_{L^{1}\left(0, T ; L^{r}\left(\Omega ; L^{1}(D)\right)\right)}+$ $\left\|\frac{\partial \varphi}{\partial t}\right\|_{L^{1}\left(0 ; T ; L^{1}(\Omega \times D)\right)}$, where $r>1$ if $d=2$ or $r=\frac{6}{5}$ if $d=3$.

Remark 3.10. Although we have introduced smoothing through the parameter $\alpha>0$ into the model $\left(\mathrm{P}_{\alpha}\right)$ compared to the standard polymer model, $\left(\mathrm{P}_{0}\right)$, we wish to stress that in both the corotational and the noncorotational case the bounds on $\sim_{\alpha}$, the variable of real physical interest, in (3.92a) are independent of this smoothing parameter $\alpha$.

3.3. Existence for $\left(\mathbf{P}_{\varepsilon}\right)$ in the corotational case. Finally, in the corotational case we can pass to the limit $\alpha \rightarrow 0$ in $\left(\mathrm{P}_{\alpha, \varepsilon}\right)$ with $J_{\alpha}^{x}$ replaced by $S_{\alpha}$ to obtain a weak formulation of $\left(\mathrm{P}_{\varepsilon}\right)$. Hence, recalling Remark 3.7, we require $\partial \Omega \in C^{2}$ to obtain the existence result, and the bounds $(3.89 \mathrm{a}, \mathrm{b})$, for $\left(\mathrm{P}_{\alpha, \varepsilon}\right)$ with $J_{\alpha}^{x}$ replaced by ${\underset{\sim}{\alpha}}_{\alpha}$. As the bounds $(3.89 \mathrm{a}, \mathrm{b})$ are independent of $\alpha$ in the corotational case, we obtain immediately the following lemma.

Lemma 3.11. Let $\partial \Omega \in C^{2}$ and $\underset{\approx}{\sigma}(\cdot)=\underset{\widetilde{\sigma}}{\omega}(\cdot)$. Then there exists a subsequence of $\left\{u_{\alpha, \varepsilon}, \psi_{\alpha, \varepsilon}\right\}_{\alpha}$, and functions $u_{\varepsilon} \in L^{\infty}\left(0, T ; L_{\sim}^{2}(\Omega)\right) \cap L^{2}(0, T ; V) \cap W^{1, \frac{4}{d}}\left(0, T ; V_{\sim}^{\prime}\right)$ and $\psi_{\varepsilon} \in L^{2}\left(0, T ; X^{+}\right)$with $M^{-\frac{1}{2}} \psi_{\varepsilon} \in L^{\infty}\left(0, T ; L^{2}(\Omega \times D)\right)$, such that, as $\alpha \rightarrow 0$,

$$
\begin{aligned}
\frac{\psi_{\alpha, \varepsilon}}{M^{\frac{1}{2}}} & \rightarrow \frac{\psi_{\varepsilon}}{M^{\frac{1}{2}}} & \text { weak* in } L^{\infty}\left(0, T ; L^{2}(\Omega \times D)\right), \\
M^{\frac{1}{2}} \underset{\sim}{\nabla_{q}}\left(\frac{\psi_{\alpha, \varepsilon}}{M}\right) & \rightarrow M^{\frac{1}{2}} \underset{\sim}{\nabla_{q}\left(\frac{\psi_{\varepsilon}}{M}\right)} & \text { weakly in } L^{2}\left(0, T ; \underset{\sim}{\left.L^{2}(\Omega \times D)\right),}\right. \\
M^{\frac{1}{2}}{\underset{\sim}{\nabla}}_{x}\left(\frac{\psi_{\alpha, \varepsilon}}{M}\right) & \rightarrow M^{\frac{1}{2}} \underset{\sim}{\nabla_{x}\left(\frac{\psi_{\varepsilon}}{M}\right)} & \text { weakly in } L^{2}\left(0, T ; \underset{\sim}{\left.L^{2}(\Omega \times D)\right),}\right. \\
\underset{\approx}{C}\left(\psi_{\alpha, \varepsilon}\right) & \rightarrow \underset{\approx}{C}\left(\psi_{\varepsilon}\right) & \text { weak* in } L^{\infty}\left(0, T ; \underset{\sim}{\left.L^{2}(\Omega)\right),}\right.
\end{aligned}
$$

and

$$
\begin{aligned}
& {\underset{\sim}{u}, \varepsilon}_{\alpha} \rightarrow{\underset{\sim}{u}}_{\varepsilon} \quad \text { weak }{ }^{*} \text { in } L^{\infty}\left(0, T ; L_{\sim}^{2}(\Omega)\right), \\
& u_{\alpha, \varepsilon} \rightarrow u_{\varepsilon} \quad \text { weakly in } L^{2}(0, T ; V), \\
& {\underset{\sim}{\gamma}}_{\gamma} \frac{\partial \tilde{\sim}_{\alpha, \varepsilon}}{\partial t} \rightarrow \underset{\sim}{S_{\gamma}} \frac{\partial \tilde{\sim}_{\varepsilon}}{\partial t} \quad \text { weakly in } L^{\frac{4}{d}}(0, T ; V) \text {, } \\
& u_{\alpha, \varepsilon} \rightarrow u_{\varepsilon} \quad \text { strongly in } L^{2}\left(0, T ; L_{\sim}^{r}(\Omega),\right.
\end{aligned}
$$

where $r \in[1, \infty)$ if $d=2$ and $r \in[1,6)$ if $d=3$.

In addition, we have that

$$
\sup _{t \in(0, T)}\left[\int_{\Omega}\left|u_{\varepsilon}\right|^{2} \mathrm{~d} x\right]+\nu \int_{0}^{T} \int_{\Omega}\left|\nabla_{x} \underset{\sim}{u_{\varepsilon}}\right|^{2} \mathrm{~d} x \underset{\sim}{ } \mathrm{d} t \leq C(T),
$$$$
\sup _{t \in(0, T)}\left[\int_{\Omega \times D} \frac{\left|\psi_{\varepsilon}\right|^{2}}{M} \mathrm{~d} \underset{\sim}{\mathrm{d}} \mathrm{d} x\right]+\int_{0}^{T} \int_{\Omega \times D}\left[\varepsilon M\left|\nabla_{x}\left(\frac{\psi_{\varepsilon}}{M}\right)\right|^{2}+\frac{1}{\lambda}\left|\nabla_{q}\left(\frac{\psi_{\varepsilon}}{M}\right)\right|^{2}\right] \mathrm{d} \underset{\sim}{q} \mathrm{~d} \underset{\sim}{\mathrm{d}} \mathrm{d} t
$$

$$
+\sup _{t \in(0, T)}\left[\int_{\Omega}\left|\underset{\sim}{C}\left(\psi_{\varepsilon}\right)\right|^{2} \mathrm{~d} x\right]+\int_{0}^{T}\left\|\underset{\sim}{S_{\gamma}} \frac{\partial \tilde{\sim}_{\varepsilon}}{\partial t}\right\|_{H^{1}(\Omega)}^{\frac{4}{d}} \mathrm{~d} t \leq C(T) .
$$


It follows immediately from $(3.96 \mathrm{~d}),(3.4 \mathrm{a}, \mathrm{b})$, and $(3.97 \mathrm{a})$ that

$$
\underset{\sim}{S} \underset{\sim}{u} \alpha, \varepsilon \rightarrow \underset{\sim}{u} \varepsilon_{\varepsilon} \quad \text { strongly in } L^{2}\left(0, T ; \underset{\sim}{L^{2}}(\Omega)\right) \quad \text { as } \alpha \rightarrow 0 .
$$

Next, we note that for all $\underset{\sim}{v} \in \underset{\sim}{H}{ }_{0}^{1}(\Omega)$ and $\underset{\sim}{\eta} \in H^{1}\left(\Omega ; L^{2}(D ; M)\right)$

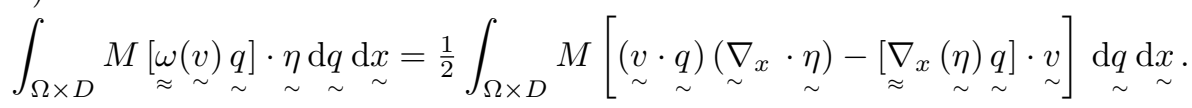

It is now a simple matter to prove existence of a solution to the following problem: $\left(\mathrm{P}_{\varepsilon}\right)$ Find $u_{\varepsilon} \in L^{\infty}\left(0, T ; L_{\sim}^{2}(\Omega)\right) \cap L^{2}(0, T ; \underset{\sim}{V}) \cap W^{1, \frac{4}{d}}\left(0, T ; V_{\sim}^{\prime}\right)$ and $\psi_{\varepsilon} \in L^{2}(0, T$; $X)$, with $M^{-\frac{\tilde{1}}{2}} \psi_{\varepsilon} \in L^{\infty}\left(0, T ; L^{2}(\Omega \times D)\right)$ and $\underset{\approx}{C}\left(\psi_{\varepsilon}\right) \in L^{\infty}\left(0, T ; \underset{\sim}{L^{2}}(\Omega)\right)$, such that $u_{\varepsilon}(\cdot, 0)=u_{0}(\cdot)$ and

$$
\begin{aligned}
& \int_{0}^{T}\left\langle\frac{\partial u_{\varepsilon}}{\partial t}, \underset{\sim}{w}\right\rangle \mathrm{d} t+\int_{0}^{T} \int_{\Omega}\left[\left[\left(u_{\varepsilon} \cdot \nabla_{x}\right) u_{\varepsilon}\right] \cdot \underset{\sim}{w}+\nu \underset{\approx}{\nabla_{x}} \underset{\sim}{u_{\varepsilon}}:{\underset{\sim}{\nabla}}_{x} \underset{\sim}{w}\right] \mathrm{d} x \underset{\sim}{\mathrm{d}} t
\end{aligned}
$$

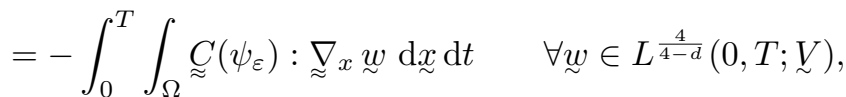

$-\int_{0}^{T} \int_{\Omega \times D} \frac{\psi_{\varepsilon}}{M} \frac{\partial \varphi}{\partial t} \mathrm{~d} \underset{\sim}{\mathrm{d}} \mathrm{d} x \mathrm{~d} t-\int_{\Omega \times D} \frac{\psi_{0}(\cdot, \cdot)}{M} \varphi(\cdot, \cdot, 0) \mathrm{d} \underset{\sim}{q} \mathrm{~d} x$

$$
\begin{aligned}
& +\int_{0}^{T} \int_{\Omega \times D}\left[\varepsilon M \underset{\sim}{\nabla_{x}}\left(\frac{\psi_{\varepsilon}}{M}\right)-{\underset{\sim}{u}}_{\varepsilon} \psi_{\varepsilon}\right] \cdot \nabla_{x}\left(\frac{\varphi}{M}\right) \mathrm{d} \underset{\sim}{q} \mathrm{~d} \underset{\sim}{x} \mathrm{~d} t \\
& +\int_{0}^{T} \int_{\Omega \times D} \frac{M}{2 \lambda} \underset{\sim}{\nabla_{q}}\left(\frac{\psi_{\varepsilon}}{M}\right) \cdot \underset{\sim}{\nabla_{q}}\left(\frac{\varphi}{M}\right) \mathrm{d} \underset{\sim}{q} \mathrm{~d} \underset{\sim}{x} \mathrm{~d} t \\
& +\frac{1}{2} \int_{0}^{T} \int_{\Omega \times D}\left[\underset{\approx}{\nabla_{x}}\left(\psi_{\varepsilon} \nabla_{q}\left(\frac{\varphi}{M}\right)\right) \underset{\sim}{q}\right] \cdot{\underset{\sim}{u}}_{\varepsilon} \mathrm{d} \underset{\sim}{q} \mathrm{~d} x
\end{aligned}
$$

$$
-\frac{1}{2} \int_{0}^{T} \int_{\Omega \times D}\left(\underset{\sim}{u_{\varepsilon}} \cdot \underset{\sim}{q}\right)\left[\underset{\sim}{\nabla_{x}} \cdot\left(\psi_{\varepsilon} \nabla_{\sim}\left(\frac{\varphi}{M}\right)\right)\right] \mathrm{d} q \underset{\sim}{\mathrm{d} x} \underset{\sim}{x}=0 \quad \forall \varphi \in \mathcal{Y},
$$

where $\mathcal{Y}$ is defined as the completion of $C_{0}^{\infty}((-T, T) ; \mathcal{K})$ in the norm $\|\cdot\|_{\mathcal{Y}}$ defined by

$$
\begin{array}{r}
\|\varphi\|_{\mathcal{Y}}:=\|\varphi\|_{L^{\frac{4}{4-d}}\left(0, T ; X_{q}\right)}+\left\|\mid \underset{\sim}{|q|} M^{\frac{1}{2}}{\underset{\sim}{q}}_{q}\left(\frac{\varphi}{M}\right)\right\|_{L^{r}\left(0 ; T ; H^{1}\left(\Omega ; L^{2}(D)\right)\right)} \\
+\left\|M^{-\frac{1}{2}} \frac{\partial \varphi}{\partial t}\right\|_{L^{1}\left(0 ; T ; L^{2}(\Omega \times D)\right)}
\end{array}
$$

with $r>2$ if $d=2$ or $r=4$ if $d=3$. This, in particular, implies that each $\varphi \in \mathcal{Y}$ satisfies $\varphi(\cdot, \cdot, T)=0$.

Noting $(3.96 \mathrm{a}-\mathrm{d})$ and $(3.95 \mathrm{~d})$, we can pass to the limit $\alpha \rightarrow 0$ in (3.21a) in the corotational case to obtain (3.100a). As ${\underset{\sim}{\alpha}}_{\alpha} u_{\alpha, \varepsilon} \in L^{2}(0, T ; \underset{\sim}{V})$, we can apply (3.99) to (3.88) with $\underset{\sim}{v}={\underset{\sim}{\alpha}}_{\alpha} \underset{\sim}{u} \alpha, \varepsilon$ and $\underset{\sim}{\eta}=\frac{\psi_{\alpha, \varepsilon}}{M} \nabla_{q}\left(\frac{\varphi}{M}\right)$ and then use (3.95a-d), (3.96d), and (3.98) to pass to the limit $\alpha \rightarrow 0$ to obtain (3.100b). 
Finally, we note that, for any $s \in(0, T)$ and $\Delta t$ sufficiently small such that $0<\Delta t<s$, we can choose $\varphi(\underset{\sim}{x} \underset{\sim}{q}, t)=\frac{1}{\Delta t}\left\{[s-t]_{+}-[s-\Delta t-t]_{+}\right\} M(\underset{\sim}{q})$ in $\left(\mathrm{P}_{\varepsilon}\right)$ to yield that

$$
\frac{1}{\Delta t} \int_{s-\Delta t}^{s} \int_{\Omega \times D} \psi_{\varepsilon}(\underset{\sim}{x} \underset{\sim}{q}, t) \mathrm{d} \underset{\sim}{q} \mathrm{~d} \underset{\sim}{\mathrm{d}} \mathrm{d} t=\int_{\Omega \times D} \psi_{0}(\underset{\sim}{x} \underset{\sim}{q}) \mathrm{d} \underset{\sim}{q} \mathrm{~d} \underset{\sim}{x} .
$$

Passing to the limit $\Delta t \rightarrow 0$, we deduce that

$$
\int_{\Omega \times D} \psi_{\varepsilon}(\underset{\sim}{x}, \underset{\sim}{q}, s) \mathrm{d} \underset{\sim}{q} \mathrm{~d} \underset{\sim}{x}=\int_{\Omega \times D} \psi_{0}(\underset{\sim}{x} \underset{\sim}{q}) \mathrm{d} \underset{\sim}{q} \mathrm{~d} \underset{\sim}{x} \quad \forall s \in(0, T) .
$$

Identical statements can be made about $\psi_{\alpha}$ in $\left(\mathrm{P}_{\alpha}\right)$ and $\psi_{\alpha, \varepsilon}$ in $\left(\mathrm{P}_{\alpha, \varepsilon}\right)$.

Acknowledgment. We are grateful to Lingyun Zhang (School of Mathematical Sciences, Beijing University) for his careful reading of the manuscript.

\section{REFERENCES}

[1] L. Ambrosio, Transport equation and Cauchy problem for BV vector fields, Invent. Math., 158 (2004), pp. 227-260.

[2] J. W. Barrett, Ch. Schwab, And E. Süli, Existence of global weak solutions for some polymeric flow models, Math. Models Methods Appl. Sci., 15 (2005), pp. 939-983.

[3] A. V. Bhave, R. C. Armstrong, and R. A. Brown, Kinetic theory and rheology of dilute, nonhomogeneous polymer solutions, J. Chem. Phys., 95 (1991), pp. 2988-3000.

[4] P. Biller and F. Petruccione, The flow of dilute polymer solutions in confined geometries: A consistent numerical approach, J. Non-Newtonian Fluid Mech., 25 (1987), pp. 347-363.

[5] R. Bird, C. Curtiss, R. Armstrong, and O. Hassager, Dynamics of Polymeric Liquids, Volume 2: Kinetic Theory, John Wiley and Sons, New York, 1987.

[6] P. Constantin, Nonlinear Fokker-Planck Navier-Stokes systems, Commun. Math. Sci., 3 (2005), pp. 531-544.

[7] C. F. Curtiss, R. B. Bird, And O. Hassager, Kinetic theory and rheology of macromolecular solutions, Adv. Chem. Phys., 35 (1976), pp. 31-117.

[8] R. J. DiPerna And P.-L. Lions, Ordinary differential equations, transport theory and Sobolev spaces, Invent. Math., 98 (1989), pp. 511-547.

[9] Q. Du, C. LIU, AND P. YU, FENE Dumbbell model and its several linear and nonlinear closure approximations, Multiscale Model. Simul., 4 (2005), pp. 709-731.

[10] W. E, T. J. LI, AND P.-W. Zhang, Well-posedness for the dumbbell model of polymeric fluids, Comm. Math. Phys., 248 (2004), pp. 409-427.

[11] A. W. El-Kareh And L. G. LeAL, Existence of solutions for all Deborah numbers for a nonNewtonian model modified to include diffusion, J. Non-Newtonian Fluid Mech., 33 (1989), pp. 257-287.

[12] C. Foias, D. D. Holm, And E. S. Titi, The Navier-Stokes- $\alpha$ model of fluid turbulence, Phys. D, 152/153 (2001), pp. 505-519.

[13] V. Girault and P.-A. Raviart, Finite Element Methods for Navier-Stokes Equations, Springer Ser. Comput. Math. 5, Springer-Verlag, Berlin, 1986.

[14] B. Jourdain, T. Lelièvre, And C. Le Bris, Numerical analysis of micro-macro simulations of polymeric fluid flows: A simple case, Math. Models Methods Appl. Sci., 12 (2002), pp. 1205-1243.

[15] B. Jourdain, T. Lelièvre, And C. Le Bris, Existence of solution for a micro-macro model of polymeric fluid: The FENE model, J. Funct. Anal., 209 (2004), pp. 162-193.

[16] R. Keunings, A survey of computational rheology, in Proceedings of the XIIIth International Congress on Rheology, Vol. 1, British Society of Rheology, Cambridge, UK, 2000, pp. 7-14.

[17] T. LI, H. Zhang, AND P.-W. Zhang, Local existence for the dumbbell model of polymeric fluids, Comm. Partial Differential Equations, 29 (2004), pp. 903-923.

[18] P.-L. Lions And M. Masmoudi, Global solutions for some Oldroyd models of non-Newtonian flows, Chinese Ann. Math. Ser. B, 21 (2000), pp. 131-146.

[19] A. LozInski, Spectral Methods for Kinetic Theory Models of Viscoelastic Fluids, Thèse No. 2860 (2003), École Polytechnique Fédérale de Lausanne, Lausanne, Switzerland, 2003. 
[20] A. Lozinski, C. Chauvière, J. Fang, And R. G. Owens, Fokker-Planck simulations of fast flows of melts and concentrated polymer solutions in complex geometries, J. Rheol., 47 (2003), pp. 535-561.

[21] A. Lozinski, R. G. Owens, And J. FAng, A Fokker-Planck-based numerical method for modelling non-homogeneous flows of dilute polymeric solutions, J. Non-Newtonian Fluid Mech., 122 (2004), pp. 273-286.

[22] H.-C. Öttinger, Stochastic Processes in Polymeric Fluids, Springer-Verlag, Berlin, 1996.

[23] F. Отto and A. Tzavaras, Continuity of Velocity Gradients in Suspensions of Rod-Like Molecules, SFB Preprint 147, Institute of Applied Mathematics, University of Bonn, Bonn, Germany, 2004.

[24] F. Petruccione And P. Biller, A consistent numerical analysis of the tube of flow of dilute polymer solutions, J. Rheol., 32 (1988), pp. 1-21.

[25] M. RENARDY, An existence theorem for model equations resulting from kinetic theories of polymer solutions, SIAM J. Math. Anal., 22 (1991), pp. 313-327.

[26] J. D. SchieBer, Generalized Brownian configuration field for Fokker-Planck equations including center-of-mass diffusion, J. Non-Newtonian Fluid Mech., 135 (2006), pp. 179-181.

[27] J. D. Schieber And H. C. Öttinger, The effects of bead inertia on the Rouse model, J. Chem. Phys., 89 (1988), pp. 6972-6981.

[28] R. Temam, Navier-Stokes Equations. Theory and Numerical Analysis, 3rd ed., Stud. Math. Appl. 2, North-Holland, Amsterdam, 1984.

[29] H. Triebel, Interpolation Theory, Function Spaces, Differential Operators, 2nd ed., Johann Ambrosius Barth, Heidelberg, 1995.

[30] P. YU, Q. DU, AND C. LiU, From micro to macro dynamics via a new closure approximation to the FENE model of polymeric fluids, Multiscale Model. Simul., 3 (2005), pp. 895-917.

[31] H. Zhang and P. Zhang, Local existence for the FENE-dumbbell model of polymeric fluids, Arch. Ration. Mech. Anal., 181 (2006), pp. 373-400. 